\title{
Surveys of ultraviolet-excess quasar candidates in large fields ${ }^{\star}$
}

\section{The area around NGC 450}

\author{
E. Gosset ${ }^{1, \star \star}$, O. Moreau ${ }^{2,1}$, J. Surdej ${ }^{1,3, \star \star \star}$, J.-P. Swings ${ }^{1}$, and H.C. Arp ${ }^{4,5}$ \\ 1 Institut d'Astrophysique, Université de Liège, Avenue de Cointe 5, B-4000 Liège, Belgium \\ 2 Centre d'Analyse des Images et DEMIRM, Observatoire de Paris, 61 avenue de l'Observatoire, F-75014 Paris, France \\ 3 Space Telescope Science Institute, Homewood Campus, 3700 San Martin Drive, Baltimore MD 21218, U.S.A. \\ 4 In illo tempore, Mount Wilson and Las Campanas Observatories of the Carnegie Institution of Washington, U.S.A. \\ 5 Max-Planck-Institut für Astrophysik, Karl-Schwarzschild-Straße 1, D-85740 Garching bei München, Germany
}

Received December 1, 1995; accepted October 18, 1996

\begin{abstract}
We describe the results of a survey for moderately bright quasars performed in a 20.6-square-degree field around the galaxy NGC 450. The quasar candidates were selected on the basis of their ultraviolet excess: by comparative visual inspection of the double image of each single object on a $U / B$ dual-exposure Schmidt photographic plate, 95 primary and 45 secondary quasar candidates were selected on the basis of their $U$ image being too bright. The spectroscopic identification of the primary candidates led to the discovery of 59 bona fide quasars (out of which 6 were previously known). The Palomar Schmidt plate was digitised using the MAMA measuring machine and the outcoming data reduced using ad hoc procedures. A photometric calibration allowed us to derive values for the limiting magnitudes and for the $U-B$ index selection threshold of the survey. A catalogue containing 60 quasars is presented with accurate positions, magnitudes and additional information such as redshifts. We studied the spatial distribution of the objects and detected, for the quasars of our sample, a significant deviation from randomness in the form of a propensity to cluster in pairs on the celestial sphere with a typical scale of about 10 arcmin. We also formally detected a tendency towards
\end{abstract}

Send offprint requests to: E. Gosset

* Based on observations acquired at the Mount Palomar and Las Campanas Observatories as well as at the European Southern Observatory. Also based on Schmidt-plate digitisations performed with the MAMA measuring machine of C.A.I. (I.N.S.U., Paris).

** Chercheur Qualifié au Fonds National de la Recherche Scientifique (Belgium).

$\star \star \star$ Presently member of the Astrophysics Division, Space Science Department of the European Space Agency and also Directeur de Recherches au Fonds National de la Recherche Scientifique (Belgium). a 3-D clustering, but this result is induced by a single pair of quasars. A forthcoming paper will deal with a similar work performed in a field around NGC 520; the latter field is located directly to the North of the present one and slightly overlaps it.

Key words: quasars: general — surveys

\section{Introduction}

Several fields $\left(\simeq 5^{\circ} \times 5^{\circ}\right)$ near selected bright, possibly disturbed, galaxies (NGC 5334, NGC 450, NGC 520, ...) as well as far from them, have been searched for quasar candidates on the basis of the presence of an ultraviolet excess (more precisely a $U / B$ excess). This work was initiated several years ago (Arp \& Surdej 1982; Surdej et al. 1982) and status reports may be found in Gosset et al. (1986) and Swings et al. (1988). Initially, this project was triggered by the need for further observational evidence supporting the uniform distribution of quasars, their possible location in superclusters (Oort 1981; Oort et al. 1981; Oort 1983), or in the vicinity of irregular bright galaxies and/or nearby the companions thereof (Arp 1981; Arp 1983; Arp \& Duhalde 1985; Arp 1987). Thanks to their high luminosity, quasars are the landmarks of the far Universe. They constitute one of the rare tracers of matter at very large distances. Quasars should outline some characteristics of the Universe, such as the distribution of matter (at least the luminous one) at mid- to large-scales. If the existence of a clustering up to a typical scale of $10 \mathrm{~h}^{-1} \mathrm{Mpc}$ seems now established (Iovino et al. 1991; Shanks et al. 1987), the question is still a matter of debate for the largest scales. The signal 
for clustering in excess of randomness is usually considered as non-existent at large scales but the detections of three different quasar superstructures $\left(100-200 \mathrm{~h}^{-1} \mathrm{Mpc}\right)$ have been reported in the literature (Webster 1982; Crampton et al. 1987, 1989; Clowes \& Campusano 1991a). A fourth possible case is presented in Graham et al. (1995). Some authors even suggested that all the small-scale clustering be localized in such superstructures (Crampton et al. 1989; Mo \& Fang 1993; Clowes \& Campusano 1991b). The study of this kind of clustering is of the highest interest and is a strong observational constraint situated, for quasars, somewhere between the structures found locally in the different maps of galaxies (as reported, for example, by de Lapparent et al. 1988 and by Geller \& Huchra 1989 and usually corresponding to a redshift $z \lesssim 0.1$ ) and the region of relevance of the observations of the cosmic microwave background. The latter is rather consistent with a Universe without strong pristine structures (Smoot et al. 1992; see also Partridge 1995). The possible existence of quasar superstructures is therefore extremely constraining for the different theories of the Universe. Such studies are however difficult due to the relative rarity of these interesting objects. Quasars are also interesting because they can be considered as beacons in the Universe, and their luminosity can be exploited to analyse the intervening absorbing matter lying between the quasars and us. They allowed the discovery of the $\operatorname{Ly} \alpha$ forest clouds which remain one of the most disturbing constituents of the Universe (Sargent et al. 1980; Sargent \& Boksenberg 1983; Smette et al. 1992; Borgeest 1993; Smette 1994). Almost as a by-product, damped Ly $\alpha$ and Mg II systems, usually associated with intervening galaxies, are now inducing a reappraisal of the dimension of these dynamical systems which are suspected to be much more extended than previously thought (Bergeron \& Boissé 1991; Aragón-Salamanca et al. 1994). Comparative studies of the absorbed spectra of a close pair (be it true or just apparent) of quasars is a tool to derive sizes of the intervening clouds (see Smette et al. 1992; Smette 1994; Smette et al. 1995). Finally, the deep nature of quasars is still something which is not fully understood. Several questions related to their evolution and to the origin of their high luminosity remain unanswered (Weedman 1986; Netzer 1989; Blandford 1989; Krolik 1989; Begelman 1989; Antonucci 1993). In order to improve our knowledge of the Universe, of its structure and of its content, as well as of the physics of quasars, it is evident that we need larger and larger samples in order to statistically increase the range of observed properties. On one side, this will induce a better knowledge of these interesting objects and, on the other side, this will increase the probability to detect strange, marginal objects that could shed new light on the subject. Furthermore, the discovery of a large number of quasars may be a source for new gravitational lens systems, objects presenting a high cosmological interest (see e.g. the review by Refsdal \& Surdej 1994). Quasars are easily characterized on the basis of their spectra but a systematic spectrography at even moderate resolution of every object in a wide region of the sky would be exaggerately telescope-time consuming. A preliminary selection of quasar candidates in wide fields is thus necessary in order to significantly reduce the amount of objects to observe spectroscopically. For this reason, we initially performed in a few fields a selection of quasar candidates on the basis of the $U / B$ excess criterion. However, this kind of survey contains well-known caveats, the most famous being the cut-off at $z \simeq 2.3$ corresponding to the appearance of the $\operatorname{Ly} \alpha$ forest in the $U$ band and of the relevant emission line in the $B$ one. Besides this well-known effect, other causes of incompleteness exist. An example is the presence of the Mg II emission line $(\lambda 2800 \AA)$ in the $B$ band for $z \simeq 0.7-0.8$ (Wampler \& Ponz 1985). For these reasons, it is necessary to cover the same areas with other survey techniques in order to improve the density of known quasars and to achieve a level of completeness as high as possible. One field (around NGC 520) has already been covered by an objective-prism survey (see Gosset et al. 1990). Most of the fields are also partly covered by a deep grism survey (see a preliminary report by Gosset et al. 1991). We also initiated more sophisticated multicolour UBVRI surveys (see a first brief report by Moreau et al. 1994). These other techniques are also addressing different populations of quasars, particularly through the range of accessible redshifts. In order to reach our aims concerning the density of known objects and the completeness, it is clear that multitechnique surveys are essential to attain a high level of efficiency at any redshift. Therefore, we decided to search all our selected fields with the largest possible diversity of techniques (adding, as criteria, the absence of proper motion $^{1}$, variability, X-ray emission, etc.). Each of these isolated techniques represents a large amount of work and a detailed account of the individual results is necessary in order to later investigate and efficiently compare all the techniques which were used. In the present paper, we report on the $U / B$ excess technique as applied in a field around the galaxy NGC 450 - a galaxy possibly anomalous (Arp 1987, Moles et al. $1994)$-. This is a rather old survey initiated more than fifteen years ago. Although we are currently working on the different extensions, we prefer to publish the $U / B$ survey separately both for practical and historical reasons, thus preserving its homogeneity. In Sect. 2, we describe the survey itself and the selection of the quasar candidates. Section 3 deals with the spectroscopic and photometric observations necessary to the project whereas Sect. 4 is devoted to the astrometric and photometric calibrations. Section 5 details a few results of the present quasar survey and Sect. 6 contains considerations on the spatial

\footnotetext{
Although gravitational lensing could in some cases mimic small proper-motion effects.
} 
distribution of the discovered quasars. Section 7 concludes the present paper.

\section{The $U / B$ survey for quasar candidates}

A photographic plate of our selected field located near NGC 450 was obtained in 1979 with the 48-inch Schmidt telescope of the Mount Palomar Observatory. The plate covers the unvignetted part of the Schmidt telescope field and corresponds to a $4.54 \times 4.54$ square. It was first exposed through the $B$ band during 7 minutes, then slightly offset and exposed through the $U$ band during 60 minutes. We thus got a $U / B$ dual-exposure plate. The exposure times were calibrated in such a way that a faint object with $U-B \simeq-0.4$ gives rise to two images of similar brightness. Bluer objects have a $U$ image brighter than the $B$ one and the selection of quasar candidates on the basis of a $U / B$ excess can easily be done by detecting these comparatively bright $U$ images. The advantage of using a dual $U / B$ plate is that the selection does not suffer from the inhomogeneities in the response of the emulsion since both the $U$ and $B$ images are very close together: the $U-B$ index threshold is therefore not expected to strongly vary across the plate. Table 1 presents further information on that plate on which our survey is based.

Table 1. Characteristics of photographic plate PS26671

\begin{tabular}{ll}
\hline Plate number & PS26671 \\
Field centre (B1950) & $\alpha_{1950}=01^{\mathrm{h}} 16^{\mathrm{m}} 05^{\mathrm{s}} .3$ \\
& $\delta_{1950}=-01^{\circ} 08^{\prime} 25^{\prime \prime}$ \\
Field centre (J2000) & $\alpha_{2000}=01^{\mathrm{h}} 18^{\mathrm{m}} 38^{\mathrm{s}} \cdot 6$ \\
& $\delta_{2000}=-00^{\circ} 52^{\prime} 40^{\prime \prime}$ \\
& $4.54 \times 4.54$ \\
Area covered & $(20.6$ square degrees $)$ \\
& Kodak $103 \mathrm{a}-\mathrm{O}$ \\
Emulsion & baking \\
Hypersensitization & UG1 $10 \times 10$ \\
$U$-band filter & GG13 \\
$B$-band filter & 60 minutes \\
Exposure time in $U$ band & 7 minutes \\
Exposure time in $B$ band & 12 arcsec W-E \\
$U / B$ offset & Nov. $14-15,1979(1979.872)$ \\
Epoch & Palomar 48 in. Schmidt \\
Telescope & H.C.A. \\
Observer & 67.2 arcsec $/ \mathrm{mm}$ \\
Angular scale & \\
\hline \hline
\end{tabular}

The plate has been visually inspected on an $X Y$ table equipped with a binocular (magnification factor of 30). In order to improve the objectivity of the survey, the plate has been systematically studied by two different persons without any preliminary concertation. Each person slowly scanned the plate twice and rescanned it a third time in order to attribute to each candidate a complex quality factor giving information on the magnitude of its $U-B$ excess and on its brightness. Subsequently, the two lists were merged: in general the agreement between both lists was rather good but certainly not perfect. The candidates from the two lists have been arranged into three classes according to the associated quality factors and to the presence of the object in one or two of the lists.

We came out with 140 candidates, 95 primary and 45 secondary ones. A third class exists (tertiary candidates) which contains objects that cannot be considered as good candidates on the sole basis of the present survey. Preliminary coordinates of the 140 candidates have been measured on a modified Zeiss blink comparator located in Liège and on the Optronics measuring machine of the European Southern Observatory (ESO) at Garching bei München. They have been converted to equatorial coordinates for subsequent observations.

\section{Observations}

\subsection{Spectroscopic observations}

Our sample of primary quasar candidates has been observed spectroscopically in order to identify the nature of the candidates. Several observing runs have been necessary; they are labelled A to E and are listed in Table 2 along with additional information on the utilized instrumentation. Wavelength calibrations were essentially done by comparison with He-Ar spectra (sometimes also $\mathrm{Hg}$ spectra) acquired with the telescope in the same position as for the science exposures. Flat fields were also obtained a few times during the night. All the observations were reduced in the standard way (nevertheless taking into account the particularities of the different instruments) using the IHAP reduction package developed at ESO (see Middelburg 1985). The response curves were derived from the observation of classical spectrophotometric standard stars (e.g. L930-80, HZ 4, ...).

\subsection{Photometric observations}

A faint photometric sequence in the Johnson $U B V$ system has been established in the field of interest in order to allow a precise calibration of the plate. Three observing runs of standard photometry have been necessary to cover all our main fields; further information is given in Table 3. The Las Campanas run has been determining for building-up the faint end of the sequences. Our program stars have been observed against the standard stars catalogued by Landolt (1983). The data have been first reduced night by night, then merged and reduced again with a fixed colour transformation. We made use of the SNOPY software initially developed at ESO by R. Barbier. 
Table 2. Information about the different observing runs for the spectroscopic identification of the primary quasar candidates

\begin{tabular}{|c|c|c|c|c|c|}
\hline Label & $\mathrm{A}$ & B & $\mathrm{C}$ & $\mathrm{D}$ & $\mathrm{E}$ \\
\hline \multirow[t]{2}{*}{ Observing dates } & October & December & September & November & September $27-$ \\
\hline & $1-2,1981$ & $19-21,1981$ & $16-20,1982$ & $10-24,1982$ & October 4,1983 \\
\hline Observer & H.C.A. & H.C.A. & J.S. & H.C.A.+J.P.S. & H.C.A. \\
\hline \multirow[t]{2}{*}{$\overline{\text { Telescope }}$} & Palomar & Palomar & $\mathrm{ESO}$ & Las Campanas & Las Campanas \\
\hline & 200 in. & 200 in. & $3.6 \mathrm{~m}$ & 100 in. & 100 in. \\
\hline Instrument & $\begin{array}{l}\text { Double Spectr. }^{(1)} \\
+ \text { Intens. Reticon }\end{array}$ & $\begin{array}{l}\text { Double Spectr. }^{(1)} \\
+ \text { Intens. Reticon }\end{array}$ & $\begin{array}{l}\mathrm{B} \& \mathrm{C}+ \\
\mathrm{IDS}^{(2)}\end{array}$ & $\begin{array}{c}\text { B \& C + } \\
\text { Shectograph }^{(3)}\end{array}$ & $\begin{array}{c}\text { B \& C + } \\
\text { Shectograph }^{(3)}\end{array}$ \\
\hline Grating (grooves/mm) & 600 & 600 & 300 & 600 & 600 \\
\hline Reciprocal dispersion $(\AA / \mathrm{mm})$ & 72 & 72 & 224 & 114 & 114 \\
\hline Detector format & 3744 & 3744 & 2048 & 3744 & 3744 \\
\hline Pixel size $(\check{A})$ & 1 & 1 & 4.5 & 1 & 1 \\
\hline Aperture on the sky $(\operatorname{arcsec})$ & $2.5 \varnothing$ & $2.5 \varnothing$ & $4 \times 4$ & $2 \times 2$ & $2 \times 2$ \\
\hline Wavelength domain $(\AA)$ & $3200-5900$ & $3200-5900$ & $3800-7300$ & $3300-7100$ & $3300-7100$ \\
\hline Resolution ( $\AA$, FWHM) & 6 & 6 & 12 & 4 & 4 \\
\hline
\end{tabular}

1 Oke \& Gunn (1982).

2 Intensified Dissector Scanner (Danks 1983).

3 The Shectograph is described in Shectman (1981).

Table 3. Information about the three observing runs of standard photometry

\begin{tabular}{lcccc}
\hline Dates & Useful nights & Telescope & Instrument & Observer \\
\hline October 27 - November 7, 1983 & 8 & ESO $1 \mathrm{~m}$ & standard one-channel photometer & $(1)$ \\
October 13 - October 21, 1984 & 8 & Las Campanas $100 \mathrm{in}$. & standard one-channel photometer \\
November 23 - November 30, 1984 & 5 & ESO $1 \mathrm{~m}$ & J.S. + J.P.S. & standard one-channel photometer \\
\hline \hline
\end{tabular}

1 See Danks (1983).

2 With, as photometer, an RCA C31034A GaAs tube.

\section{Astro/photometric calibration of the survey}

In order to accurately determine the limiting magnitude of the survey and the $U-B$ threshold actually applied to the selection of quasar candidates, as well as to measure the positions and magnitudes of the candidates, we digitised, using MAMA, the dual-exposure Schmidt plate PS26671 in small fields surrounding each candidate.

\subsection{The MAMA digitising machine}

MAMA (Machine Automatique à Mesurer pour l'Astronomie) is a high-speed and high-accuracy microdensitometer. It is operated by the Centre d'Analyse des Images (C.A.I.) located at Observatoire de Paris (Guibert \& Moreau 1991). Technical characteristics of the scanning system are described elsewhere (Moreau 1992); they are summarized here:

- multichannel 1024-photodiode Reticon detector,
- 4096-grey-level quantification,

- $10 \mu \mathrm{m}$ pixel size (corresponding to 0.67 arcsecond on the plate),

- absolute astrometric accuracy $1 \mu \mathrm{m}$ (Soubiran 1992), i.e. 0.07 arcsec on the plate,

- absolute photometric accuracy of about $2 \%$.

Note that before the scan of each plate, the MAMA detector is systematically calibrated on a small area of the plate through individual gain and dark current measurements for each of the 1024 photodiodes.

\subsection{Plate scanning and object measurements}

For the measurement of the quasar candidates, MAMA was used in its pavés digitising mode (Berger et al. 1991). In this random access mode, small images are acquired around each object position given in a scanning list. The size of these pavés was chosen to be $256 \times 256$ pixels, i.e. $172 \times 172 \operatorname{arcsec}$ on the sky. For the scanned dual-exposure 
plate, each pavé contains two images of the same object: one in each of the $U$ and $B$ bands. The two images associated with each candidate were visually identified on screen displays of the MAMA frames (using the VISUAL visualization software; see Vétois 1995) in order to ensure their correct retrieval in the catalogue of detected objects.

The scanned images were digitally processed, as described in Berger et al. (1991), for object detection. The images were segmented (Rosenfeld 1969) at a fixed density value above the local sky background and we considered as an object every domain of more than 9 connected pixels whose density is above this threshold. A local value for the sky background was computed inside each of the pavés on the basis of density histograms of the pixels. The corresponding standard deviation on each pixel, $\sigma_{\mathrm{px}}$, was accordingly estimated in every pavé. The threshold for segmentation of the images was set to $2.3 \sigma_{\mathrm{SB}}$ above the local background, where $\sigma_{\mathrm{SB}}$ is the mean value on the plate of the "pixel-to-pixel" standard deviations $\sigma_{\mathrm{px}}$. One should note that a few close associations were not directly resolved by the deep segmentation we made, and that the $U$ and $B$ images of some rather bright objects, thus having a large area, did merge at this threshold. For some objects, the local background was so polluted that measurements at $2.3 \sigma$ were not correct. For all these objects, we then performed several runs of our process with increasing values of the threshold (up to $5.0 \sigma_{\mathrm{SB}}$ ) to correctly extract them. On the opposite, a few very faint objects were not detected at $2.3 \sigma_{\mathrm{SB}}$; in such a case, we also segmented the MAMA images at $2.0 \sigma_{\mathrm{SB}}$.

The object detection process supplies a catalogue whose main entries are $(X, Y)$ machine coordinates of the objects, their area (number of pixels inside the segmented contour), density flux (sum of the density values of the pixels above local background, expressed in MAMA quantification levels), local background value and morphological parameters including the ellipse axis parameters of the contour. The photometric and astrometric reduction of the produced object catalogues was conducted separately in each band using the procedure detailed in Berger et al. (1991); the main steps are displayed hereafter.

\subsection{Photometric reduction}

Dual-exposure plates are not well adapted to accurate machine photometric measurements. Indeed, they are noisy because $U$ and $B$ backgrounds are superimposed on the same emulsion. Moreover, the type of emulsion of the plate we used (103a-O) worsens the problem. However, we found essential to measure the magnitudes of the candidates on the plate actually used for their selection. Thus, the photometric accuracy on the $U$ and $B$ magnitudes presented hereafter is not the best we can achieve on Schmidt plates digitised with MAMA (see for example Moreau \& Reboul 1995). Improved magnitudes, derived for other epochs, will be available for objects of the field thanks to the system- atic $U B V R I$ multicolour survey we are conducting (see Moreau et al. 1994).

In order to calibrate the photographic plate, we used the specially acquired photometric sequence mentioned in Sect. 3.2. The stars of this sequence were included in the list of objects to scan in addition to the quasar candidates. The brighter stars cannot be used to calibrate dualexposure plates, because the two images of these objects merge. They were however useful as intermediate standards during the set-up of the sequence.

The determination of the magnitudes of the candidates was performed through a $\log$ (flux)/magnitude calibration curve derived from a fit over the standard stars (where flux is the density flux defined in Sect. 4.2). The adopted polynomial calibration fits are displayed in Fig. 1 and we give in Table 4 further information: the domain of validity, the number of standard stars used and the obtained residuals (standard deviation of the fit). These residuals give an estimate of the photometric accuracy achieved, at least in non-peripheral regions of the plate and for objects brighter than about magnitude 19 . We estimate that our $U$ and $B$ magnitude measurements are subject to a global standard deviation of about 0.15 mag. A photometric reduction has also been performed for each of the other segmentation thresholds that were used. It should be clear that the combination of the filters (see Table 1) and of the emulsion only gives a poor approximation of the standard $U$ and $B$ bands. Thus, the present calibration is an approximation which is not fully correct, particularly for the case of quasars. No effort has been made to compute continuum (i.e. corrected for lines) magnitudes.

Table 4. Photometric calibration

\begin{tabular}{ccccc}
\hline Band & Pol. order & Mag. range & Residuals & Stars used \\
\hline$U$ & 3 & $14.1-19.5$ & $0.10 \mathrm{mag}$ & 26 \\
$B$ & 4 & $14.1-19.8$ & $0.10 \mathrm{mag}$ & 29 \\
\hline \hline
\end{tabular}

\subsection{Astrometric reduction}

Bright objects, as usually are the astrometric standard stars, see their two images merged on dual-exposure plates. Performing high-precision astrometry on such a plate turned out to be extremely difficult. Therefore, we measured the positions of the candidates on another plate, namely ESO8799. This classical single-exposure $B$ plate, obtained at ESO in the framework of our ongoing $U B V R I$ systematic survey, was also scanned in the pavés mode. The astrometric reduction was done using a transformation from $(X, Y)$ plate coordinates to $(\xi$, $\eta)$ standard coordinates, through an adjusted third order 
Table 5. Measurement repeatability tests on the $U$ and $B$ images of the faint object \#243 (see Sect. 4.5)

\begin{tabular}{lccccc}
\hline & $X$ & $Y$ & $(X, Y)$ position & Area $(10 \mu$ m-pixels $)$ & Flux \\
\hline Standard deviation in $U$ & $0.7 \mu \mathrm{m}$ & $1.5 \mu \mathrm{m}$ & $1.6 \mu \mathrm{m}$ & 2.1 & $4.9 \%$ \\
Maximal departure from mean in $U$ & $2.1 \mu \mathrm{m}$ & $2.2 \mu \mathrm{m}$ & $2.8 \mu \mathrm{m}$ & 5.2 & $6.7 \%$ \\
Standard deviation in $B$ & $0.3 \mu \mathrm{m}$ & $0.5 \mu \mathrm{m}$ & $0.6 \mu \mathrm{m}$ & 0.5 & $2.0 \%$ \\
Maximal departure from mean in $B$ & $0.4 \mu \mathrm{m}$ & $0.7 \mu \mathrm{m}$ & $0.8 \mu \mathrm{m}$ & 0.7 & $3.7 \%$ \\
\hline \hline
\end{tabular}

(a) U exposure on Palomar plate PS26671

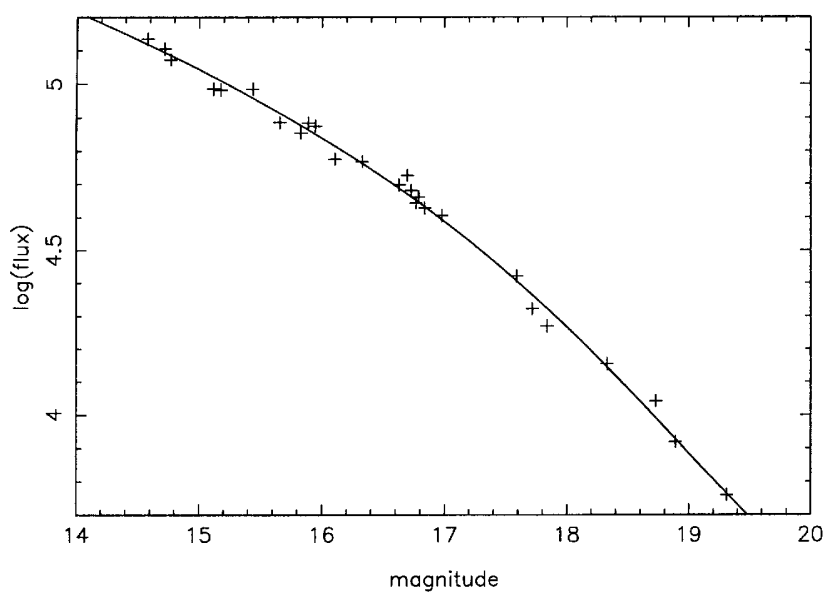

(b) B exposure on Polomar plate PS26671

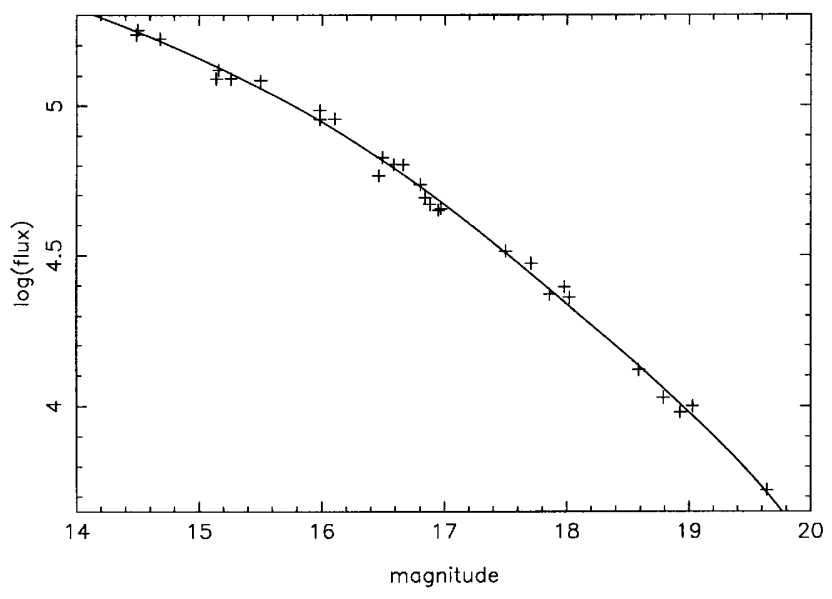

Fig. 1. Photometric calibration curves for each of the $U$ and $B$ exposures

bidimensional polynomial, and then to $(\alpha, \delta)$ equatorial coordinates, through the classical inverse operation of the gnomonic projection. The 20 coefficients of the polynomial (the plate constants) were determined using propermotion-corrected positions of 84 reference stars from the PPM star catalogue (Röser \& Bastian 1991), expressed in standard coordinates via the gnomonic projection. The residuals on the transformation were 0.34 arcsec (standard deviation of the fit referring to one coordinate). The positions of the objects lying close to the edges of the field are expected to be measured less precisely; globally, the achieved astrometric accuracy for these is estimated to be 0.5 arcsec. Note that a few very faint objects were not recovered on the ESO plate; we give for them a slightly less precise position determined on the dual-exposure Palomar plate.

\subsection{Repeatability tests on MAMA measurements}

The repeatability of the measurement chain (MAMA digitisation + object detection) was tested in extreme conditions, i.e. on a faint object measured on a noisy plate. In order to estimate the measurement internal errors, we successively scanned on the dual-exposure plate 12 times the same object, thus taking and analysing 12 different MAMA frames. We chose a faint object: \#243 (a tertiary candidate at $U=18.6$ and $B=19.2$ ), so that the derived errors were not expected to be optimistic. The results are displayed in Table 5 where we give both the standard deviation and the maximal departure from the mean for the following quantities: $X$ and $Y$ plate coordinates of the object, its area and density flux. Object-position fluctuations have also been derived from the dispersions of $X$ and $Y$ combined via a distance calculation.

By inspecting the data, it is clear that fluctuations in position measurements are essentially related to fluctuations in the number of pixels of the object, and correspond to the passage, because of noise, of one or more pixels above or below the segmentation threshold. Obviously, the more an object has a large area i.e. - for star-like objects - is bright, the smaller are these fluctuations. On the contrary, flux fluctuations occur even with a constant number of pixels, because the density value of each pixel is affected by noise. In any case, the measured internal errors are rather small compared to the absolute accuracy we derived above. The standard deviations in flux correspond, after photometric reduction, to standard deviations of $0.05 \mathrm{mag}$ in $U$ and $0.02 \mathrm{mag}$ in $B$, whereas the standard deviations in $(X, Y)$ position correspond on the plate to $0.11 \operatorname{arcsec}$ in $U$ and $0.04 \operatorname{arcsec}$ in $B$. 


\section{Results of the survey}

\subsection{The spectroscopic follow-up of the candidates}

Out of the 95 primary candidates, 91 have been observed spectroscopically in the framework of the present survey. Among the 4 unobserved primary candidates (\#10, \#189, \#14 and \#26), one is an already known quasar (\#189 $\equiv$ Q0116 - 021). Two of the observed primary candidates remain candidates because the acquired spectra are not of sufficiently good quality to allow definitive identification (\#118 and \#179). Therefore, we identified the nature of $90(89+1)$ candidates out of 95 . Among these 90 objects, we found

- 59 quasars, out of which 2 have spectra similar to those of Seyfert 1 galaxies (among the 59 quasars, only 6 were already known from either the Parkes or the University of Michigan surveys),

-26 stars,

-2 probable stars,

- 2 Seyfert 2 galaxies or $\mathrm{H}$ II extragalactic regions,

- 1 very probable Seyfert 2 galaxy.

The efficiency of the technique for selecting quasars is around 60 to $65 \%$ for the primary candidates. It is not expected to be dramatically less for the secondary candidates but we did not intend to observe these last objects without further clues on their nature. However, one of the 45 secondary candidates has been observed and it turned out to be a quasar: \#107 $\equiv$ Q0111 - 008 .

The $U / B$ excess technique is very good at picking out the majority of the moderately low-redshift quasars hidden in a field. However, we find symptomatic that the survey that provided the largest absolute density of such quasars (Boyle et al. 1987, 1990) was also the one with the lowest efficiency $(\simeq 38 \%)$. Any attempt to improve the completeness of the survey is bound to a strong loss of efficiency. The only alternative is to perform a multitechnique survey for quasars.

The spectra of most of the active galactic nuclei of the present survey are illustrated in Fig. 2. Although the spectra have been reduced following classical methods for the correction of the atmospheric extinction and for the calibration with standard stars, the flux scale must be considered as totally relative, owing to the various circumstances of the observations such as the seeing, the climatic conditions and so on. We are sorry that a few spectra acquired during $\mathrm{A}$ and $\mathrm{B}$ runs have not been archived and are thus no longer available.

\subsection{The catalogue of quasar candidates}

We collected in Table 6 all the 140 candidates in order of increasing right ascension. The first two columns give the right ascension and the declination of the objects for the J2000 equinox (a colon indicates slightly less precise values). The third column displays the redshift of the iden- tified extragalactic objects (see Sect. 5.3 for the determination of the redshift). A colon indicates that the redshift is somewhat uncertain because only one line is present in the acquired spectrum; a question mark indicates very uncertain values. The fourth column gives either the B1950 name of the quasar (e.g. Q0107-025A), or an indication on the nature of the object (if it is not a quasar, e.g. STAR), or simply states that the object is still an unidentified candidate $(\mathrm{C})$. The fifth indicates whether the object is a primary $(\mathrm{P})$ or a secondary $(\mathrm{S})$ candidate; an asterisk is printed when the spectrum of the object is available in Fig. 2. The sixth column contains the internal identification number of the candidate in the present survey (e.g. \#30 or NGC 450 \# 30; an asterisk refers to a note in Table 7) whereas the seventh reports the identification of the candidate in the NGC 520 survey (e.g. NGC 450 \# 30 三 NGC 520 \# 173B; see also Surdej et al., in preparation). The three next columns show the $B$ and $U$ magnitudes of the objects and their $U-B$ colour indices (rounded to the nearest $0.05 \mathrm{mag}$ ). A colon indicates uncertain values for particular or very faint objects (less than $0.5 \mathrm{mag}$ above the calibration limits); for these three columns, an over-estimate or an under-estimate is given when a value could not be measured because lying out of the calibration range. The last column deals with an identification of the spectroscopic runs as well as with the exposure times in seconds (dual exposures have been acquired each time). Finding charts for the extragalactic objects are given in the Appendix.

\subsection{Information derived from the spectra}

Table 8 contains the observed air wavelengths of the different emission lines for most of the detected extragalactic objects. The positions have been measured through the fitting of a gaussian onto the observed profile, and then corrected for the Doppler effect resulting from the revolution of the Earth around the Sun and from a galactic rotation velocity, at the distance of the Sun, of $250 \mathrm{~km} \mathrm{~s}^{-1}$. The errors in position are given in the form of \pm 1 standard deviation, the latter being essentially representative of the internal consistency of our measurements. Table 8 also gives for each object the redshift as derived from the observed lines (the computations were rigorously performed on the vacuum wavelengths); the final value of $z$ and its standard deviation are based on those lines seemingly free of any vitiating effect (the lines actually used are indicated in bold characters). Vertical bars indicate the observed part of the spectrum in the quasar reference frame. The laboratory wavelength at rest of the $\mathrm{Si}$ IV/O IV] blend is taken to be $\lambda 1401.62 \AA$ according to Wills \& Netzer (1979). The rest laboratory wavelength of the $\mathrm{C}$ III] intercombination line is taken to be $\lambda 1910.11 \AA$ when the redshift is larger or equal to 1.8 and $\lambda 1907.64 \AA$ in the other cases; these values were proposed by Wills (1980) and Ferland (1981) to take into account the possible evolution of the observed 

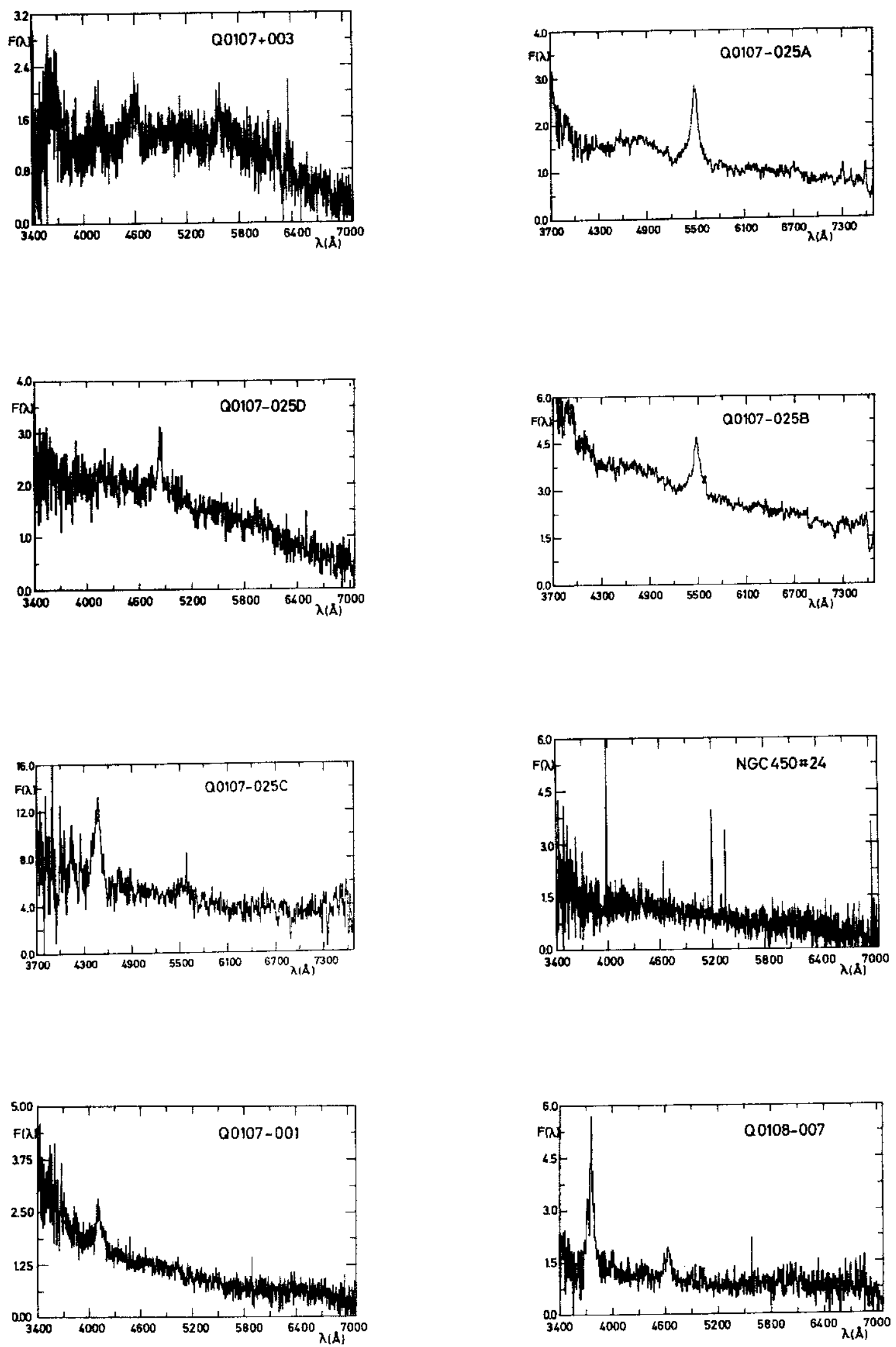

Fig. 2. Spectra of the extragalactic objects of the present survey (see also Table 6 and Sect. 5.2); the name of each object is given. The abscissae represent the wavelength expressed in $\AA$ whereas the ordinates give the flux in arbitrary units 

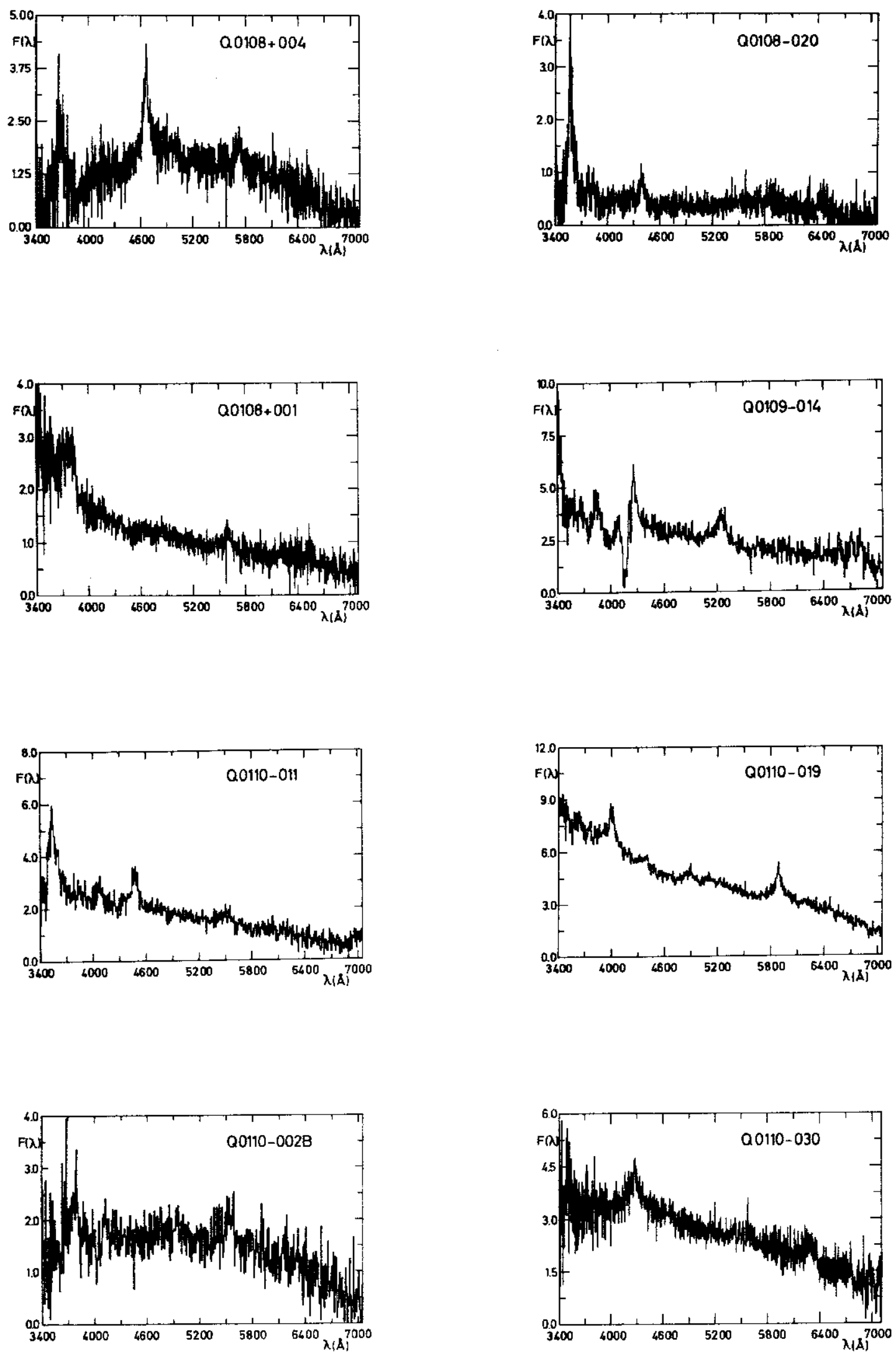

Fig. 2. continued 

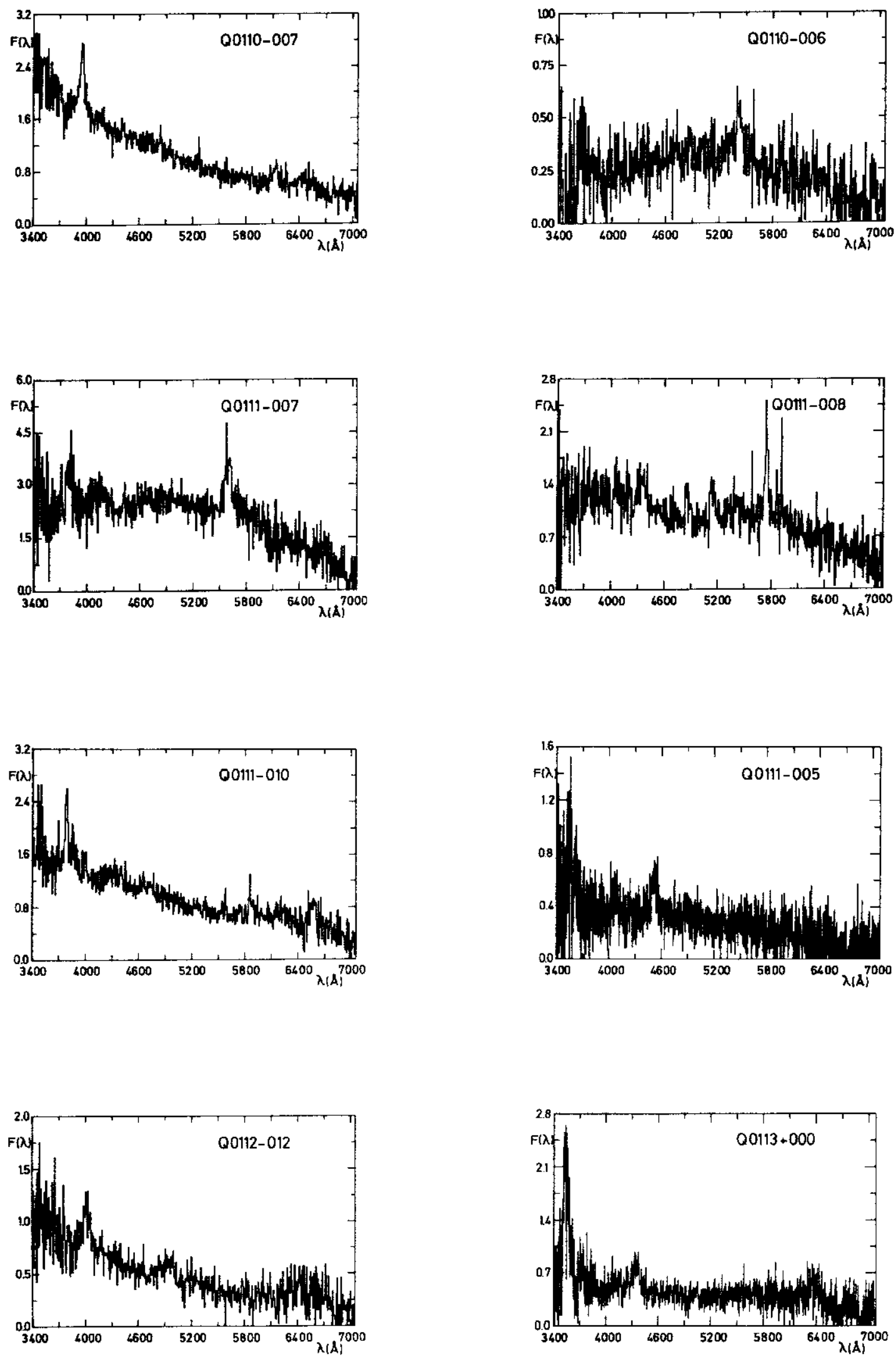

Fig. 2. continued 

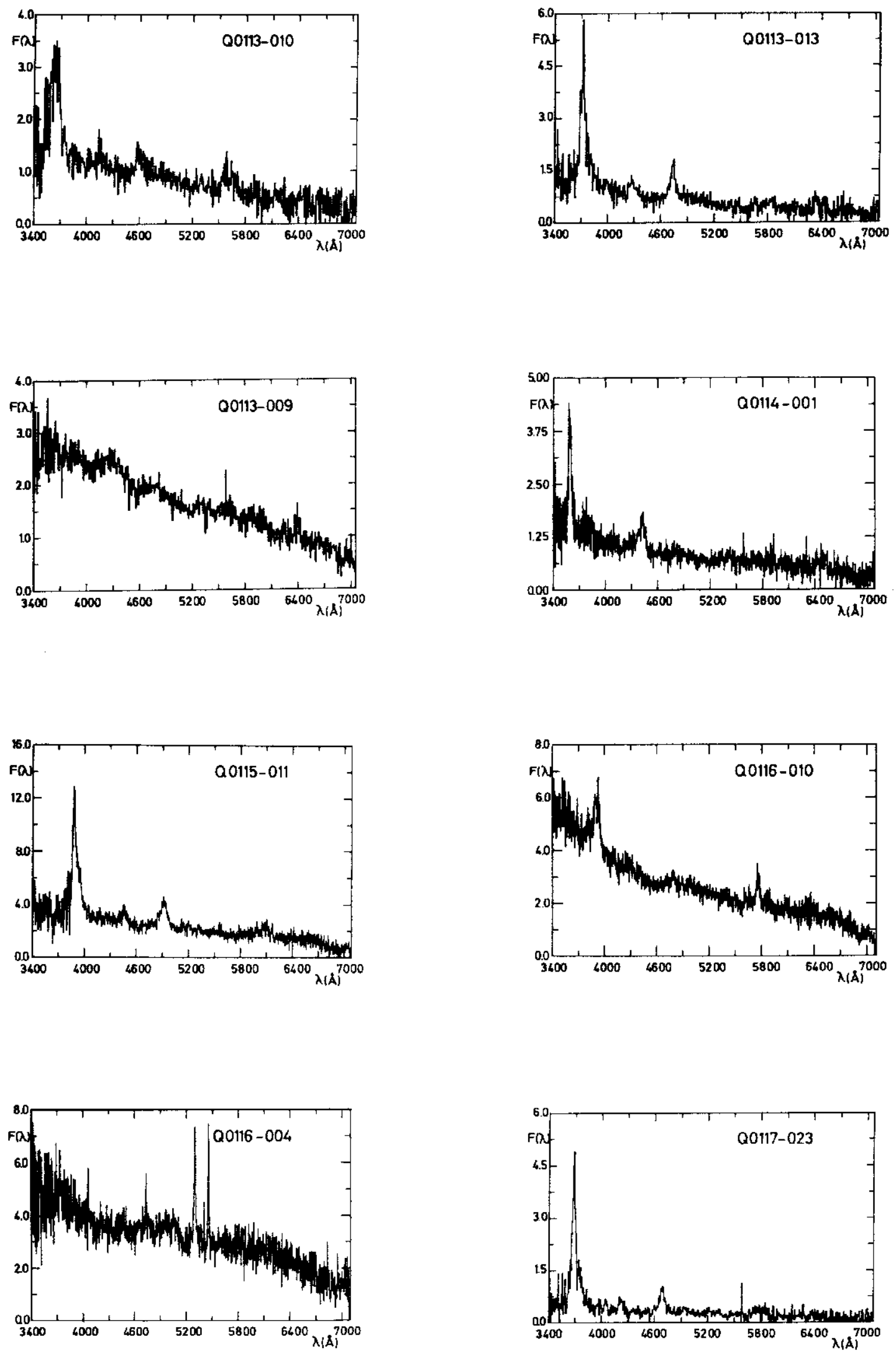

Fig. 2. continued 

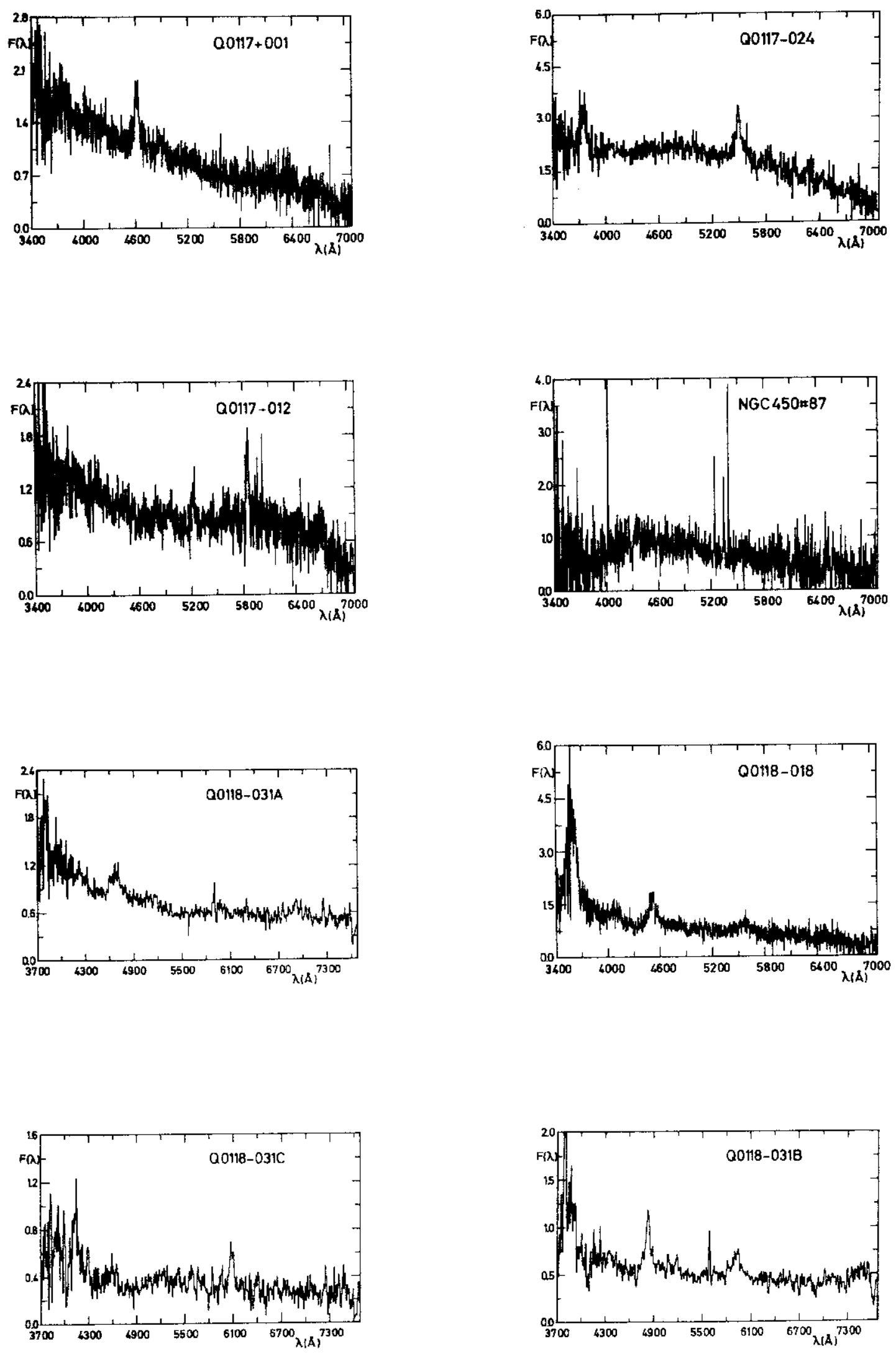

Fig. 2. continued 

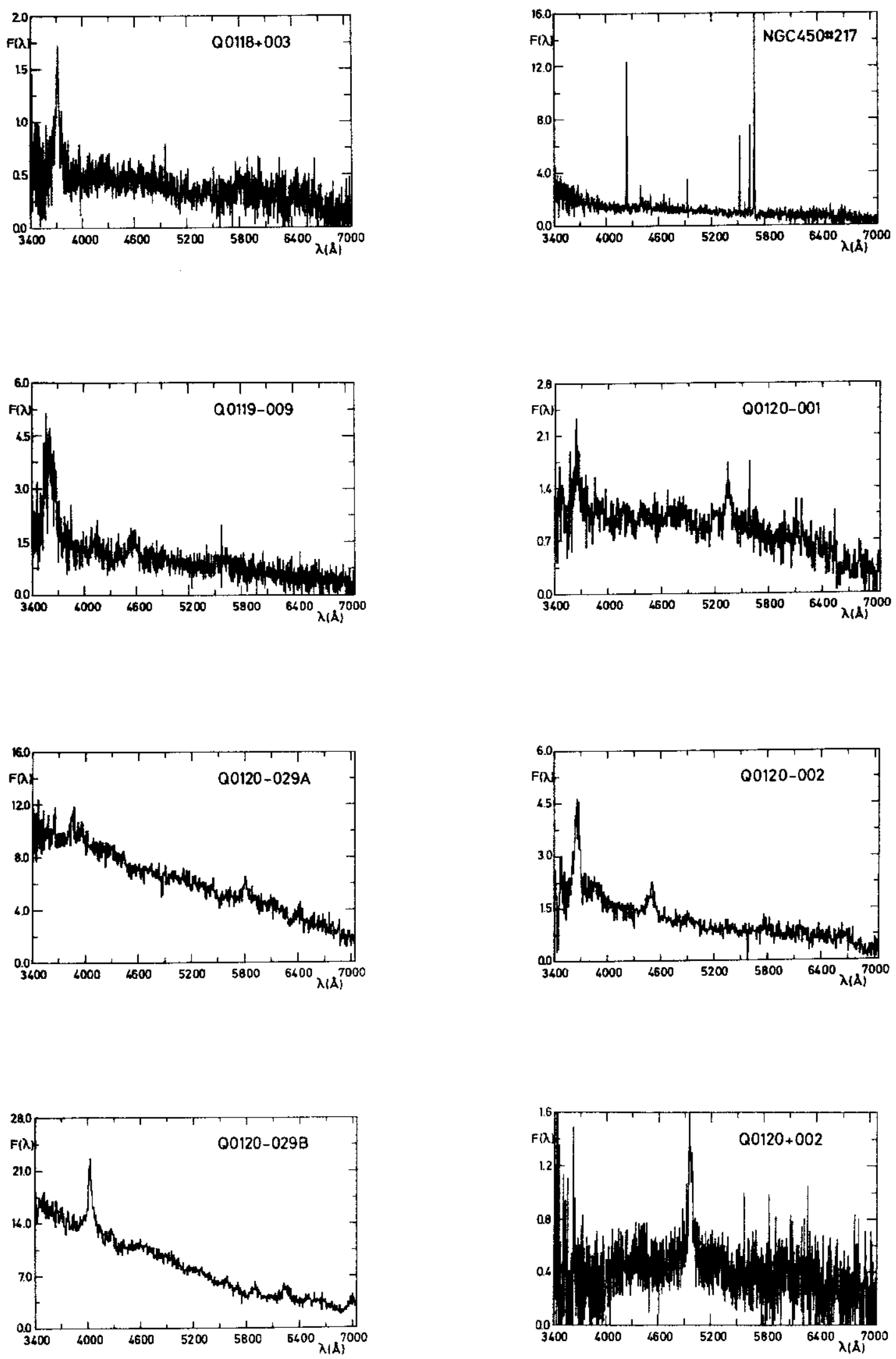

Fig. 2. continued 

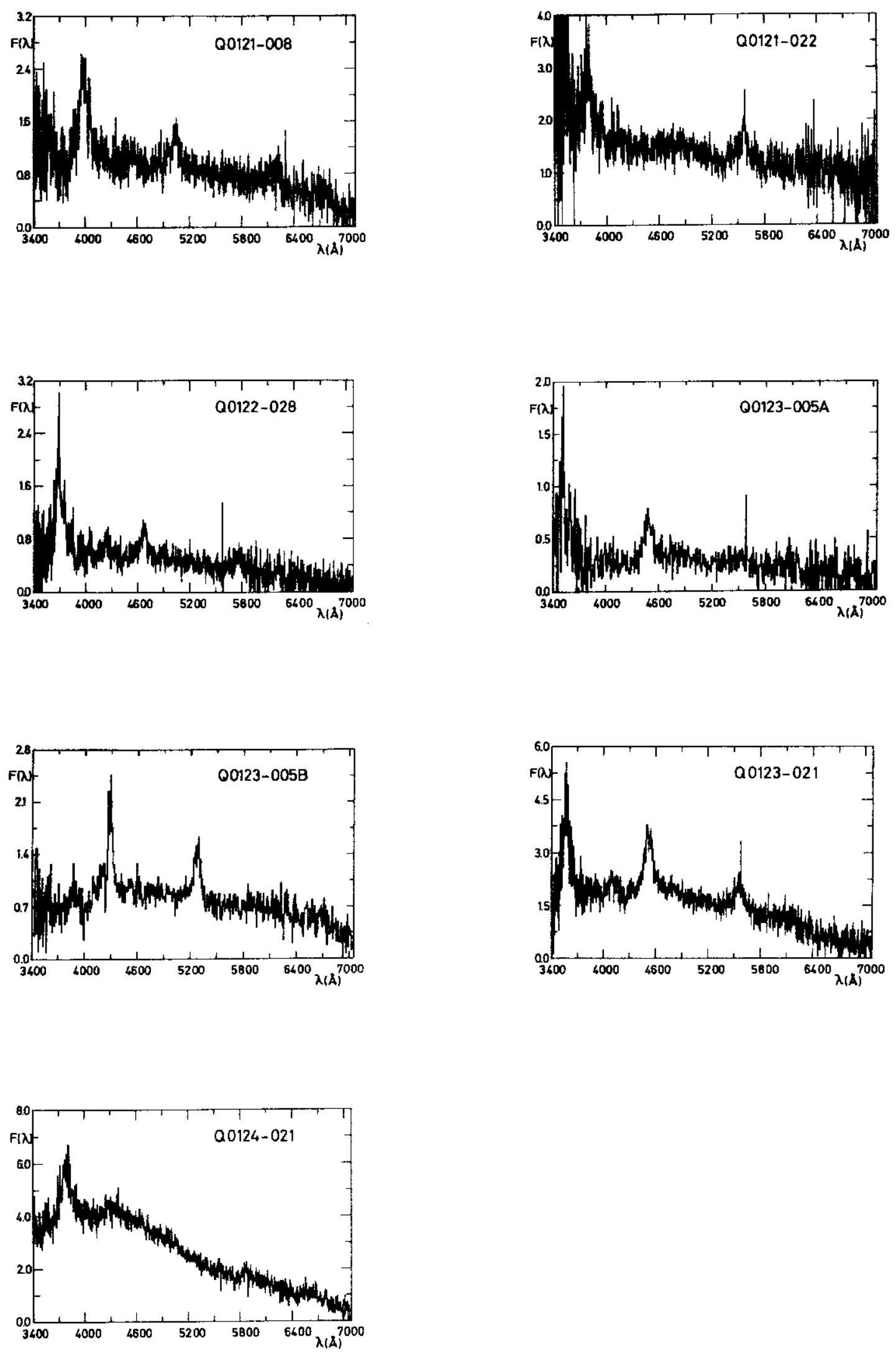

Fig. 2. continued 
Table 6. The catalogue of quasars and quasar candidates (see Sect. 5.2 for further explanations)

\begin{tabular}{|c|c|c|c|c|c|c|c|c|c|c|c|}
\hline$\alpha(J 2000)$ & $\delta(\mathrm{J} 2000)$ & $\mathbf{z}$ & Name & $\mathrm{P} / \mathrm{S}$ & NGC450 & NGC520 & B & $\mathbf{U}$ & U-B & & eectroscopy \\
\hline 954.37 & +03812.5 & 1.968 & $\mathrm{Q} 0107+003$ & P* & $\# 027 *$ & $\# 175$ & $18.30:$ & $17.30:$ & $-1.00:$ & $\mathbf{E}$ & $1500 / 1500$ \\
\hline 959.14 & $\begin{array}{lll}-2 & 53 & 1.4\end{array}$ & & STAR & $P$ & $\# 234$ & & 16.60 & 16.30 & -0.25 & $\vec{B}$ & $500 / 500$ \\
\hline 1959.78 & $\begin{array}{lll}-2 & 26 & 31.1\end{array}$ & & C & $\mathbf{s}$ & $\# 220$ & & $>19.75:$ & 18.55 & $<-1.25:$ & & \\
\hline $110 \quad 0.14$ & $\begin{array}{l}0753.3 \\
+0\end{array}$ & & $\mathrm{C}$ & s & $\# 064^{*}$ & & $18.00:$ & 17.65: & $-0.35:$ & & \\
\hline 1100.82 : & $-138 \quad 7.9:$ & & C & $\mathbf{s}$ & $\# 172^{*}$ & & $19.60:$ & $19.40:$ & $-0.20:$ & & \\
\hline $1 \quad 103.70$ & $\begin{array}{lll}-2 & 59 & 0.6\end{array}$ & & STAR & $\mathbf{P}$ & $\# 242 *$ & & 16.85: & $16.20:$ & $-0.65:$ & B & $500 / 500$ \\
\hline 11012.49 & $\begin{array}{lll}-0 & 3 & 13.8\end{array}$ & & C & $\mathrm{s}$ & $\# 080$ & & $>19.75$ : & $19.00:$ & $<-0.80:$ & & \\
\hline $\begin{array}{lll}1 & 10 & 13.23\end{array}$ & $-2 \quad 1952.2$ & 0.960 & Q0107-025A & P* & $\# 210^{*}$ & & 17.80 & 17.15 & -0.65 & C & $1200 / 1200$ \\
\hline $\begin{array}{lll}1 & 10 & 14.51\end{array}$ & -21657.5 & 0.726 : & Q0107-025D & P* & \#208 & & 18.85 & $>19.50$ : & $>0.65:$ & D & $1000 / 1000$ \\
\hline $\begin{array}{lll}1 & 10 & 16.34\end{array}$ & $\begin{array}{lll}-2 & 18 & 50.2\end{array}$ & 0.956 & $00107-025 B$ & p* & $\# 209 *$ & & 17.60 & 17.15 & -0.45 & C & $1200 / 1200$ \\
\hline 11022.01 & +1522.6 & & C & $\mathrm{P}$ & $\# 010^{*}$ & & 18.95: & $17.85:$ & -1.15 : & & \\
\hline 11027.16 & -04146.7 & & C & $s$ & $\# 116^{*}$ & & $18.60:$ & 18.30: & $-0.30:$ & & \\
\hline $\begin{array}{lll}1 & 1030.09\end{array}$ & $-2 \quad 1453.9$ & 1.893 & Q0107-025C & P* & $\# 211$ & & $19.45:$ & 18.35 & $-1.10:$ & C & $600 / 600$ \\
\hline $\begin{array}{lll}1 & 10 & 31.03\end{array}$ & +120.7 & 0.0699 & Sy2? & P* & $\# 024 *$ & & 18.90 & 18.40 & -0.50 & E & $500 / 500$ \\
\hline 11033.48 & 830.5 & 0.468 : & Q0107-001 & p* & $\# 068$ & & 19.40: & $19.20:$ & $-0.20:$ & $\mathbf{E}$ & $1300 / 1300$ \\
\hline 11037.47 & -02742.4 & 1.424 & $00108-007$ & p* & $\# 101^{*}$ & & $>19.75$ : & 18.55 & $<-1.20$ : & $\vec{D}$ & $1200 / 1200$ \\
\hline 11038.09 & +04453.5 & 2.009 & Q0108+004 & P* & $\# 028$ & \#169 & 18.25 & 17.45 & -0.80 & $\mathbf{E}$ & $1000 / 1000$ \\
\hline 11039.61 & +01117.0 & & STAR? & $\mathrm{P}$ & $\# 069$ & & 19.15 & 18.85 & -0.35 & $\mathbf{E}$ & $500 / 500$ \\
\hline 11047.96 & $\begin{array}{lll}-2 & 15 & 24.1\end{array}$ & 1.24 & $\mathrm{Q} 0108-025$ & $\mathbf{p}$ & \#212 & & 18.10 & 17.40 & -0.65 & $\mathbf{A}$ & $500 / 500$ \\
\hline $\begin{array}{lll}1 & 10 & 49.22\end{array}$ & $\begin{array}{lll}-1 & 48 & 17.5\end{array}$ & 1.302 & Q0108-020 & p* & $\# 184 *$ & & $19.60:$ & $18.60:$ & $-1.05:$ & $\mathbf{E}$ & $1000 / 1000$ \\
\hline 11056.61 & -05624.9 & & C & $\mathrm{s}$ & $\# 139$ & & 18.75 & 18.55 & -0.25 & & \\
\hline 1110.03 & $-0 \quad 4622.9$ & & C & $s$ & $\# 125$ & & 18.25 & 18.30 & 0.05 & & \\
\hline 0.63 & +0187.0 & & STAR & $\mathrm{P}$ & $\# 052$ & & 18.85 & 18.65 & -0.20 & E & $500 / 500$ \\
\hline $111 \quad 1.41$ & -14552.6 & & C & $s$ & $\# 185^{*}$ & & 18.55: & 18.70 & 0.15 : & & \\
\hline 11124.43 & +02646.9 & 1.003 & $Q 0108+001$ & p* & \#041 & $\# 184$ & 18.50 & 18.10 & -0.45 & $\mathrm{E}$ & $1500 / 1500$ \\
\hline $\begin{array}{lll}112 & 13.23\end{array}$ & +0225.4 & & C & $s$ & $\# 054$ & & 19.20 & 19.05: & $-0.15:$ & & \\
\hline 11227.63 & $-1 \quad 1221.7$ & 1.746 & $00109-014$ & P* & $\# 153^{*}$ & & 18.05 & 17.90 & -0.20 & D & $900 / 900$ \\
\hline $\begin{array}{lll}1 & 12 & 40.27\end{array}$ & $\begin{array}{lll}-0 & 52 & 3.5\end{array}$ & 1.887 & Q0110-011 & P* & $\# 126$ & & 18.80 & 17.80 & -1.00 & D & $600 / 600$ \\
\hline 11241.19 & $\begin{array}{lll}-1 & 41 & 53.2\end{array}$ & 1.099 & $00110-019$ & P* & $\# 175$ & & 17.30 & 16.90 & -0.40 & D & $2000 / 2000$ \\
\hline 11247.59 : & $-02138.4:$ & & C & $s$ & $\# 102 *$ & & 19.55: & 19.10: & -0.45 : & & \\
\hline 11248.94 & +02310.4 & & $\mathrm{C}$ & $\mathrm{s}$ & $\# 056$ & & $>19.75$ : & 18.70 & $<-1.05:$ & & \\
\hline 11254.96 & +0312.9 & $1.23 ?$ & Q0110-002A & $\mathbf{P}$ & $\# 070^{*}$ & & 18.45 & 17.65 & -0.80 & A & $500 / 500$ \\
\hline $\begin{array}{lll}1 & 1255.86\end{array}$ & +0449.6 & 0.910 & Q0110+004 & $\mathbf{P}$ & $\# 030$ & \#173B & $>19.75$ : & $19.05:$ & $<-0.75:$ & B & $500 / 500$ \\
\hline 11258.38 & -05952.5 & & STAR & $\mathrm{P}$ & $\# 140$ & & 19.20 & 18.70 & -0.50 & D & $500 / 425$ \\
\hline $113 \quad 1.76$ & $-0 \quad 0 \quad 4.3$ & 0.968 & $00110-002 B$ & p* & $\# 082$ & & 18.55 & 17.70 & -0.85 & D & $500 / 500$ \\
\hline $\begin{array}{lll}1 & 13 & 4.89\end{array}$ & $\begin{array}{lll}-0 & 28 & 3.4\end{array}$ & & c & $s$ & $\# 103$ & & 18.70 & 18.15 & -0.55 & & \\
\hline 1136.62 & -05041.5 & & STAR & $\mathbf{P}$ & $\# 127 *$ & & 18.80: & 18.70 & $-0.10:$ & D & $1500 / 1500$ \\
\hline 113 & -24937.0 & 1.235 & $00110-030$ & P* & $\# 236$ & & 17.70 & 16.75 & -0.95 & $\mathrm{E}$ & $1000 / 1000$ \\
\hline 11310.43 & -03133.6 & 0.411 & Q0110-007 & $\mathrm{P}^{*}$ & $\# 104$ & & 18.90 & 17.95 & -0.95 & D & $1800 / 1800$ \\
\hline $\begin{array}{lll}1 & 1322.91\end{array}$ & -14050.7 & & $\mathrm{C}$ & $s$ & $\# 177$ & & 18.90 & 18.55 & -0.35 & & \\
\hline 11325.07 & +11417.3 & & C & $\mathrm{s}$ & $\# 002$ & & 17.85 & 17.70 & -0.15 & & \\
\hline 11326.65 & $\begin{array}{lll}-0 & 26 & 2.9\end{array}$ & 0.935 : & Q0110-006 & $\mathrm{P}^{*}$ & $\# 105$ & & $19.70:$ & 18.80 & -0.90 : & D & $1800 / 1800$ \\
\hline 11339.41 & $\begin{array}{lll}-0 & 30 & 9.4\end{array}$ & 0.995 & $00111-007$ & p* & $\# 106$ & & 18.95 & 18.85 & -0.10 & D & $1500 / 1500$ \\
\hline 11346.80 & +02828.6 & & STAR & $\mathbf{P}$ & $\# 244^{*}$ & $\# 183$ & $14.75:$ & $13.60:$ & $-1.15:$ & $\mathrm{E}$ & $500 / 500$ \\
\hline $\begin{array}{lll}1 & 1359.58\end{array}$ & -03248.5 & 0.181 & Q0111-008 & $S^{*}$ & $\# 107 *$ & & 18.45 & 18.05 & -0.40 & $\mathrm{D}$ & $1000 / 1000$ \\
\hline 1142.40 & -04750.9 & 0.350 & $00111-010$ & P* & $\# 129$ & & 18.95 & 18.30 & -0.65 & $\mathrm{D}$ & $1200 / 1200$ \\
\hline $\begin{array}{lll}1 & 14 & 17.23\end{array}$ & $\begin{array}{llll}-0 & 55 & 18.9\end{array}$ & & $\mathrm{C}$ & $S$ & $\# 130$ & & 18.65 & 18.40 & -0.25 & & \\
\hline 11430.68 & -01551.1 & 1.908 & $00111-005$ & p* & $\# 093$ & & 19.45: & 19.20: & $-0.30:$ & $\mathrm{E}$ & $1500 / 1500$ \\
\hline 11431.78 & - 05729.7 & & STAR & $\mathrm{P}$ & $\# 141^{*}$ & & $18.55:$ & 18.10 : & $-0.45:$ & D & $600 / 267$ \\
\hline 11441.84 & $\begin{array}{lll}-1 & 17 & 23.8\end{array}$ & & C & s & $\# 155$ & & 18.60 & 18.10 & -0.50 & & \\
\hline $\begin{array}{ll}115 \quad 3.32 \\
\end{array}$ & -03910.5 & & $\mathrm{C}$ & $\mathbf{P}$ & $\# 118^{*}$ & & $19.45:$ & 18.60 & $-0.85:$ & E & $500 / 500$ \\
\hline $\begin{array}{lll}1 & 15 & 9.29\end{array}$ & $\begin{array}{lll}-1 & 46 & 9.9\end{array}$ & & STAR & $\mathbf{P}$ & $\# 187$ & & 18.00 & 17.60 & -0.40 & A & $500 / 500$ \\
\hline $\begin{array}{lll}1 & 15 & 17.13\end{array}$ & -1274.6 & 1.37 & $00112-017$ & $\mathbf{P}$ & $\# 163^{*}$ & & 17.50 & 17.00 & -0.50 & A & $500 / 500$ \\
\hline 11529.50 & -05724.0 & 1.585 & $00112-012$ & P* & $\# 245$ & & $>19.75$ : & 18.90 & $<-0.90$ : & D & $1200 / 1200$ \\
\hline 11535.54 & +04543.9 & & $\mathrm{C}$ & $S$ & $\# 034$ & \#172 & 19.05 & 18.20 & -0.85 & & \\
\hline 11537.77 & +02028.7 & 1.279 & $00113+000$ & $\mathrm{P}^{*}$ & $\# 058$ & $\# 186$ & 19.65: & 18.90 & $-0.75:$ & E & $1000 / 1000$ \\
\hline 11547.01 & - 13754.4 & & C & $\mathbf{P}$ & $\# 179 *$ & & 18.10 : & 18.05: & $-0.05:$ & $\vec{D}$ & $600 / 100$ \\
\hline 11547.96 & $-0 \quad 47 \quad 13.3$ & 1.968 & Q0113-010 & P* & $\# 131$ & & $>19.75$ : & 19.10 : & $<-0.65$; & $\mathrm{D}$ & $600 / 600$ \\
\hline 11557.14 & +04553.4 & & STAR & $\mathbf{P}$ & $\# 035$ & & 19.00 & 18.60 & -0.40 & $\mathrm{E}$ & $500 / 1000$ \\
\hline $116 \quad 6.44$ & $\begin{array}{lll}-1 & 29 & 9.8\end{array}$ & & STAR & $\mathbf{P}$ & $\# 164$ & & 19.60: & 18.55 & $-1.05:$ & D & $600 / 150$ \\
\hline 11611.98 & $\begin{array}{lll}-1 & 2 & 24.4\end{array}$ & 2.055 & $00113-013$ & P* & $\# 142$ & & $19.60:$ & 18.75 & $-0.85:$ & D & $600 / 600$ \\
\hline 11615.53 & -04334.9 & $1.263:$ & $00113-009$ & P* & \#119 & & 18.70 & 18.35 & -0.35 & D & $1500 / 1500$ \\
\hline $\begin{array}{lll}1 & 16 & 15.63\end{array}$ & -13730.8 & & C & $s$ & $\# 180$ & & 19.45 : & 18.75 & $-0.65:$ & & \\
\hline 11633.72 & +0625.8 & 1.316 & Q0114-001 & P* & $\# 073$ & & $19.60:$ & 18.15 & $-1.50:$ & E & $1000 / 1000$ \\
\hline 11655.18 & -1266.4 & & C & $S$ & \#166 & & 18.20 & 17.95 & -0.25 & & \\
\hline $\begin{array}{lll}1 & 17 & 4.54\end{array}$ & -23650.7 & & $\mathrm{C}$ & s & $\# 228$ & & 18.85 & 18.25 & -0.60 & & \\
\hline 11720.58 & -1337.7 & & $\mathrm{C}$ & $S$ & \#144 & & $>19.75$ : & 18.50 & $<-1.30:$ & & \\
\hline 11735.00 & $\begin{array}{lll}-2 & 6 & 32.2\end{array}$ & & STAR & $\mathbf{P}$ & $\# 200$ & & 19.10 & 18.55 & -0.60 & E & $700 / 700$ \\
\hline 11739.52 & -15943.5 & & C & $S$ & $\# 199$ & & $>19.75$ : & 18.35 & $<-1.45$ : & & \\
\hline 11743.15 & +02424.6 & & $\mathrm{C}$ & $\mathbf{S}$ & $\# 060$ & & 19.50 : & 19.05 : & $-0.45:$ & & \\
\hline
\end{tabular}


Table 6. continued

\begin{tabular}{|c|c|c|c|c|c|c|c|c|c|c|c|c|c|}
\hline \multicolumn{2}{|c|}{$\alpha(\mathrm{J} 2000)$} & \multicolumn{2}{|c|}{$8(\mathrm{~J} 2000)$} & \multirow[t]{2}{*}{$\mathrm{z}$} & \multirow{2}{*}{$\frac{\text { Name }}{\text { STAR }}$} & \multirow{2}{*}{$\frac{\mathrm{P} / \mathrm{S}}{\mathrm{P}}$} & \multirow{2}{*}{$\frac{\text { NGC450 }}{\# 188}$} & \multirow[t]{2}{*}{ NGC520 } & B & $\mathbf{U}$ & U-B & & ectroscopy \\
\hline 118 & 10.33 & -156 & 612.2 & & & & & & 16.40 & 15.20 & -1.20 & C & $600 / 600$ \\
\hline 118 & 24.31 & $\begin{array}{ll}-1 & 8\end{array}$ & 831.7 & & C & $\mathbf{s}$ & $\# 157$ & & 18.75 & 18.00 & -0.70 & & \\
\hline 118 & 28.02 & -052 & 239.7 & 2.175 & Q $0115-011$ & P* & $\# 132 *$ & & 18.00 & 17.55 & -0.45 & E & $500 / 500$ \\
\hline 118 & 29.63 & -054 & 414.7 & & STAR & $\mathbf{P}$ & $\# 133$ & & $>19.75$ : & 18.95 & $<-0.80$ : & $\vec{D}$ & $1800 / 1000$ \\
\hline 118 & 29.66 : & +045 & 1549.4 : & & $\mathrm{C}$ & $\mathbf{s}$ & $\# 037^{*}$ & & 19.45 : & 18.65 & $-0.75:$ & & \\
\hline 118 & 38.62 & -154 & 421.9 & 2.05 & $Q 0116-021$ & $\mathbf{P}$ & $\# 189 *$ & & 18.70 & 17.75 & -0.95 & & \\
\hline 118 & 47.79 & $\begin{array}{ll}-0 & 1\end{array}$ & 141.7 & & STAR & $\mathbf{P}$ & $\# 085$ & & 19.25 : & 18.15 & -1.10 : & $\mathbf{E}$ & $500 / 1000$ \\
\hline 118 & 51.43 & +14 & 457.0 & & C & $\mathbf{s}$ & $\# 012$ & & 19.40 : & 19.05: & $-0.35:$ & & \\
\hline 119 & 6.81 & $\begin{array}{lll}-0 & 27\end{array}$ & 740.8 & & C & $s$ & $\# 108$ & & 17.75 & 17.20 & -0.55 & & \\
\hline 119 & 21.90 & -23 & 314.9 & & STAR & $\mathbf{P}$ & $\# 202$ & & 17.10 & 16.60 & -0.50 & B & $500 / 500$ \\
\hline 119 & 22.90 & -044 & 419.9 & 1.052 & $\mathrm{Q} 0116-010$ & $\mathrm{P}^{*}$ & $\# 120$ & & 18.40 & 18.20 & -0.20 & $\mathrm{E}$ & $1200 / 1200$ \\
\hline 119 & 29.09 & -08 & $\begin{array}{ll}839.4 \\
8\end{array}$ & 0.0904 & Q0116-004 & P* & $\# 086^{*}$ & & 18.45 & 17.85 & -0.60 & $\mathrm{E}$ & $500 / 500$ \\
\hline 119 & 32.87 & +0 & 837.2 & & $\mathrm{C}$ & $\mathbf{S}$ & $\# 075$ & & 19.35: & 19.10: & -0.30 : & & \\
\hline 119 & 36.48 & +115 & $\begin{array}{ll}514.7 \\
\end{array}$ & & C & $\mathbf{s}$ & $\# 005^{*}$ & & 17.30: & 17.15: & -0.15 : & & \\
\hline 119 & 51.78 & $\begin{array}{ll}-2 & 3\end{array}$ & 319.5 & 2.019 & Q0117-023 & $\mathrm{P}^{*}$ & $\# 203$ & & $>19.75$ : & 18.60 & $<-1.20:$ & D & $1200 / 1200$ \\
\hline 120 & 5.28 & -1 & 859.3 & & C & $\mathbf{S}$ & \#158 & & 18.80 & 18.10 & -0.65 & & \\
\hline 120 & 6.52 & -221 & 141.7 & & C & $\mathbf{S}$ & $\# 224$ & & $>19.75$ : & 18.55 & $<-1.20:$ & & \\
\hline 120 & 9.58 & $\begin{array}{ll}-2 & 3\end{array}$ & 353.5 & & STAR & $\mathbf{P}$ & $\# 204$ & & 19.55 : & 18.70 & $-0.85:$ & D & $1800 / 1800$ \\
\hline 120 & 11.04 & +020 & $0 \quad 0.0$ & & C & $\mathbf{s}$ & $\# 061$ & & 19.20 & 18.50 & -0.70 & & \\
\hline 120 & 12.20 & +027 & 3.6 & 0.649 : & Q $0117+001$ & p* & $\# 044$ & & 19.55 : & 19.45: & -0.10 : & E & $1000 / 1000$ \\
\hline 120 & 13.60 & -29 & 4.3 & 0.960 & $\mathrm{Q} 0117-024$ & $\mathrm{P}^{*}$ & \#214* & & 17.90 & 17.60 & -0.30 & D & $600 / 600$ \\
\hline 120 & 15.15 & -058 & 838.4 & 0.203 & Q $0117-012$ & p* & $\# 146 *$ & & 19.30: & 18.45 & $-0.85:$ & $\mathbf{E}$ & $1000 / 1000$ \\
\hline 120 & 16.18 & $\begin{array}{lll}-0 & 47\end{array}$ & $7 \quad 5.7$ & & STAR? & $\mathbf{P}$ & $\# 134$ & & 19.55: & 18.45 & -1.10 : & $\mathbf{E}$ & $1000 / 1000$ \\
\hline 120 & 24.03 & +14 & $4 \quad 0.6$ & & C & $\mathbf{P}$ & $\# 014^{*}$ & & $>19.75$ : & 18.50 & $<-1.25:$ & & \\
\hline 120 & 44.78 & -041 & 158.9 & & STAR & $\mathbf{P}$ & $\# 121 *$ & & 18.35 & 17.75 & -0.60 & E & $1000 / 1000$ \\
\hline 120 & 50.27 & +01 & 152.1 & 0.07816 & Sy2 or HII & p* & $\# 087$ & & $19.45:$ & 18.65 & $-0.85:$ & $\mathbf{E}$ & $500 / 500$ \\
\hline 120 & 53.25 & -252 & 217.6 & 1.445 & Q0118-031A & P* & \#239 & & 18.35 & 17.45 & -0.90 & C & $900 / 900$ \\
\hline 121 & 2.43 & $\begin{array}{ll}-0 & 28\end{array}$ & $8 \quad 10.4$ & & c & $\mathbf{S}$ & $\# 110$ & & 18.50 & 18.30 & -0.20 & & \\
\hline 121 & 8.33 & -242 & 220.7 & & C & $\mathbf{S}$ & $\# 229$ & & 19.30: & 18.35 & -0.90 : & & \\
\hline 121 & 12.10 & $\begin{array}{ll}-132 \\
32\end{array}$ & 247.9 & 1.911 & Q0118-018 & P* & $\# 168$ & & $19.45:$ & 18.10 & $-1.35:$ & $\mathrm{E}$ & $1000 / 1000$ \\
\hline 121 & 12.35 & -253 & $3 \quad 7.2$ & 1.165 & Q0118-031C & P* & $\# 241$ & & 18.45 & 17.85 & -0.60 & C & $1200 / 1200$ \\
\hline 121 & 12.41 & -252 & 243.4 & 2.112 & Q0118-031B & $\mathrm{P}^{*}$ & $\# 240$ & & 19.00 & 18.15 & -0.90 & C & $1200 / 1200$ \\
\hline 121 & 13.63 & $\begin{array}{lll}-0 & 24\end{array}$ & 431.5 & & STAR & $\mathbf{P}$ & $\# 111$ & & $19.65:$ & 18.65 & $-1.00=$ & $\mathrm{E}$ & $1000 / 1000$ \\
\hline 121 & 19.25 & -217 & 78.5 & & C & s & $\# 216$ & & 18.70 & 17.95 & -0.75 & & \\
\hline 121 & 28.12 & +034 & 451.3 & 0.328 & $Q 0118+003$ & $\mathrm{p}^{*}$ & $\# 045$ & $\# 180$ & $>19.75$ : & 18.85 & $<-0.90$ & $\mathrm{E}$ & $1500 / 1500$ \\
\hline 121 & 37.58 & $\begin{array}{ll}-2 & 1\end{array}$ & 156.5 & & STAR & $\mathbf{P}$ & $\# 205$ & & 16.65 & 16.30 & -0.35 & B & $500 / 500$ \\
\hline 121 & 46.41 & -216 & $\begin{array}{l}643.9 \\
\text {. }\end{array}$ & 0.13523 & Sy 2 or $\mathrm{HIII}$ & $\mathrm{P}^{*}$ & $\# 217$ & & 18.75 & 18.50 & -0.25 & $\mathbf{E}$ & $500 / 500$ \\
\hline 121 & 46.63 & $\begin{array}{ll}-0 & 2\end{array}$ & 229.0 & & C & $S$ & $\# 088$ & & 19.35: & 18.80 & $-0.55:$ & & \\
\hline 122 & 1.26 & -039 & 940.5 & 1.943 & Q0119-009 & $\mathrm{P}^{*}$ & $\# 122$ & & $19.60:$ & 18.15 & $-1.45:$ & $\mathbf{E}$ & $1000 / 1000$ \\
\hline 122 & 18.06 & +17 & 750.1 & & STAR & $\mathbf{P}$ & $\# 015$ & & 19.35: & 18.75 & -0.60 : & $\mathbf{E}$ & $583 / 1000$ \\
\hline 122 & 39.11 & +0 & 529.9 & 0.909 & $\mathrm{Q} 0120-001$ & $\mathrm{P}^{*}$ & \#076 & & 19.25 & 18.50 & -0.75 & D & $1500 / 1500$ \\
\hline 122 & 48.87 & -239 & 953.1 & $1.073 ?$ & Q0120-029A & P* & $\# 230^{*}$ & & 18.55 & 17.95 & -0.60 & D & $1800 / 1800$ \\
\hline 122 & 59.70 & $\begin{array}{ll}-1 & 31\end{array}$ & 16.0 & & STAR & $\mathbf{P}$ & $\# 170$ & & $>19.75$ : & 18.90 & $<-0.85:$ & E & $1000 / 1000$ \\
\hline 123 & 1.82 & +03 & 323.1 & 1.355 & Q0120-002 & P* & $\# 077$ & & 18.70 & 18.10 & -0.60 & D & $1500 / 1000$ \\
\hline 123 & 2.04 & -244 & $\begin{array}{ll}4 & 0.1\end{array}$ & 0.438 & $\mathrm{Q} 0120-029 \mathrm{~B}$ & P* & $\# 231$ & & 18.10 & 17.50 & -0.60 & D & $1800 / 1800$ \\
\hline 123 & 9.04 & +033 & 5.8 & 0.772 & $\mathrm{Q} 0120+002$ & P* & $\# 047$ & & 19.25 & 18.55 & -0.70 & E & $1300 / 1000$ \\
\hline 123 & 27.10 & +18 & 822.9 & & STAR & $\mathbf{P}$ & $\# 017$ & & 18.85 & 18.05 & -0.80 & B & $500 / 500$ \\
\hline 123 & 40.08 & +13 & 332.1 & 1.31 & $21+007$ & $\mathbf{P}$ & $\# 018$ & $\# 160$ & $19.60:$ & 18.10 & $-1.50:$ & B & $500 / 500$ \\
\hline 124 & 14.53 & +111 & 115.3 & 1.555 & Q01 & $\mathbf{P}$ & $\# 020$ & & 19.35: & 18.45 & -0.9 & B & $500 / 1000$ \\
\hline 124 & 15.56 & -033 & 318.8 & 2.252 & $\mathrm{Q} 01$ & p* & $\# 123^{*}$ & & 19.30: & 18.45 & $-0.85:$ & $\mathbf{E}$ & $2000 / 2000$ \\
\hline 124 & 18.24 & +18 & 839.6 & 2.043 & Q0121 & $\mathbf{P}$ & $\# 022$ & & 19.50: & 18.35 & $-1.20=$ & B & $500 / 500$ \\
\hline 124 & 30.73 & $\begin{array}{ll}-2 & 0\end{array}$ & 016.1 & 0.988 & & $\mathrm{P}^{*}$ & $\# 206$ & & 19. & 18.45 & -0.60 & E & $1000 / 1000$ \\
\hline 124 & 59.83 & -233 & 341.8 & 2.022 & Q $0122-028$ & P* & $\# 232$ & & $>19.75$ : & 18.65 & $<-1.15:$ & $\mathrm{E}$ & $1000 / 1000$ \\
\hline 125 & 14.85 & $\begin{array}{lll}-138 & 38\end{array}$ & 89.2 & & STAR & $\mathbf{P}$ & $\# 181^{*}$ & & 18.95 & 18.20 & -0.75 & D & $796 / 1200$ \\
\hline 125 & 24.26 & $\begin{array}{ll}-0 & 4\end{array}$ & 2.7 & & STAR & $\mathbf{P}$ & $\# 089 *$ & & 19.25 & 18.95 & -0.30 & B & $500 / 500$ \\
\hline 125 & 25.69 & +018 & $8 \quad 12.3$ & & STAR & $\mathbf{P}$ & $\# 063$ & & 19.60 : & 18.60 & $-1.00=$ & $\mathrm{E}$ & $500 / 500$ \\
\hline 125 & 38.52 & -222 & 246.6 & & C & $\mathbf{S}$ & $\# 225$ & & $>19.75$ : & 18.95 & $<-0.85:$ & & \\
\hline 125 & 44.02 & $-0 \quad 15$ & 543.6 & 1.889 & Q0123-005A & P* & $\# 097$ & & 19.00 & 18.80 & -0.20 & D & $1200 / 1200$ \\
\hline 126 & 2.22 & $\begin{array}{ll}-0 & 19\end{array}$ & 924.2 & 1.763 & Q0123-005B & $\mathrm{P}^{*}$ & $\# 099$ & & 18.45 & 17.75 & -0.70 & D & $1200 / 1200$ \\
\hline 126 & 2.56 & -048 & 834.4 & & STAR & $\mathbf{P}$ & $\# 137$ & & 17.65 & 17.15 & -0.55 & $\mathbf{E}$ & $1000 / 1000$ \\
\hline 126 & 4.21 & -026 & 651.5 & & C & $s$ & $\# 112$ & & 18.95 & 18.50 & -0.50 & & \\
\hline 126 & 4.44 & -151 & 123.8 & & C & $s$ & $\# 193$ & & $>19.75$ : & 18.75 & $<-1.00:$ & & \\
\hline 126 & 17.63 & $\begin{array}{ll}-1 & 38\end{array}$ & 844.0 & & C & $S$ & $\# 183$ & & $>19.75$ : & 18.40 & $<-1.35:$ & & \\
\hline 126 & 28.66 & +027 & 747.9 & & C & $S$ & $\# 049$ & & 18.25 & 17.90 & -0.35 & & \\
\hline 126 & 30.23 & -153 & 357.5 & 1.914 & $\mathrm{Q} 0123-021$ & $\mathrm{P}^{*}$ & $\# 194^{*}$ & & 18.40 & 17.30 & -1.10 & $\mathbf{E}$ & $1000 / 1000$ \\
\hline 126 & 46.10 & +033 & 346.5 & & c & $s$ & $\# 050$ & & 19.20 & 18.70 & -0.50 & & \\
\hline 126 & 55.96 & +052 & 232.3 & & $\mathrm{C}$ & $P$ & $\# 026^{*}$ & & 19.70: & 18.70 & $-1.00:$ & & \\
\hline 126 & 59.98 & +05 & $\begin{array}{ll}5 & 0.9\end{array}$ & & C & s & $\# 078$ & & 18.35 & 18.20 & -0.10 & & \\
\hline 127 & 1.58 & +043 & 330.2 & & C & s & $\# 040$ & & 19.20 & 19.00: & -0.20 : & & \\
\hline 127 & 8.67 & -155 & 537.9 & 0.355 & Q0124-021 & $\mathrm{P}^{*}$ & $\# 195^{*}$ & & 17.35 & 16.90 & -0.45 & E & $1000 / 1000$ \\
\hline
\end{tabular}


Table 7. Notes to the catalogue of Table 6

\begin{tabular}{|c|c|}
\hline Object & Comments \\
\hline \#005 & measured at the $5.0 \sigma$ threshold (background problem and deblending of the two exposures) \\
\hline \#010 & measured at the $4.0 \sigma$ threshold (for background and deblending), not observed spectroscopically \\
\hline \#014 & not observed spectroscopically \\
\hline \#024 & nebular image? \\
\hline \#026 & not observed spectroscopically \\
\hline \#027 & measured at the $4.0 \sigma$ threshold (background problem) \\
\hline \#037 & position from the Palomar plate (not detected on the ESO one) \\
\hline \#064 & measured at the $5.0 \sigma$ threshold (background problem) \\
\hline \#070 & quasar? \\
\hline \#086 & Seyfert 1 ? fuzzy? \\
\hline \#089 & variable? near PKS 0122 - 003 \\
\hline \#101 & galaxy present to the SW \\
\hline \#102 & position from the Palomar plate (not detected on the ESO one) \\
\hline \#107 & observed spectroscopically (although secondary candidate) because \#104 to \#106 are quasars \\
\hline \#116 & measured at the $3.0 \sigma$ threshold (deblending of the two exposures) \\
\hline \#118 & spectrum underexposed, to be reobserved \\
\hline \#121 & spectrum not recorded \\
\hline \#123 & nebula at $3^{\prime \prime}$ to the SE \\
\hline \#127 & the $B$ exposure may be corrupted \\
\hline \#132 & previously known quasar: UM 314, QSO $0115-011(z=2.19)$ \\
\hline \#141 & measured at the $3.0 \sigma$ threshold (deblending with a nearby companion) \\
\hline \#146 & Seyfert $1 ?$ nebular image? \\
\hline \#153 & BAL quasar \\
\hline \#163 & previously known quasar: PKS $0112-017$, UM 310, QSO $0112-017(z=1.365)$ \\
\hline \#172 & measured at the $2.0 \sigma$ threshold; position from the Palomar plate (not detected on the ESO one) \\
\hline \#179 & measured at the $2.0 \sigma$ threshold; to be reobserved spectroscopically, looks like a QSO at $z=0.247$ ? \\
\hline \#181 & below is an object well seen on POSS \\
\hline \#184 & measured at the $3.0 \sigma$ threshold (deblending of the two exposures) \\
\hline \#185 & the $B$ exposure may be corrupted \\
\hline \#189 & not observed spectroscopically; previously known quasar: UM 315, QSO $0116-021(z=2.05)$ \\
\hline \#194 & previously known quasar: UM 322, QSO $0123-021(z=1.93)$ \\
\hline \#195 & previously known quasar: UM 324, QSO $0124-021(z=0.35)$ \\
\hline \#209 & first discovered during run A \\
\hline$\# 210$ & first discovered during run A \\
\hline$\# 214$ & previously known quasar: UM 316, QSO $0117-024(z=0.96)$ \\
\hline \#230 & very uncertain redshift \\
\hline \#242 & measured at the $3.0 \sigma$ threshold (deblending of the two exposures) \\
\hline$\# 244$ & measured at the $4.0 \sigma$ threshold (deblending of the two exposures). \\
\hline
\end{tabular}

intensity ratio in a blend. As usual, all the wavelengths below $2000 \AA$ are given as vacuum wavelengths. For clarity reasons, Table 8 has been subdivided into three parts on the basis of the redshift range. The objects are classified within each part according to increasing right ascension, as in Table 6. A legend explaining the different symbols and additional remarks are given below the table. The redshifts from Table 8 were transferred to the catalogue of Table 6 except for the three quasars Q0118 - 031A, Q0118 - 031B and Q0118 - 031C for which better values are available from Robertson et al. (1986).

For the same objects, we give in Table 9 the full widths at the bottom of the lines and the equivalent widths in the reference frame of the quasar. Table 9 is subdivided into three parts as for Table 8. Finally, let us note that a few objects at low redshift and exhibiting narrow lines over a faint continuum are given separately in Table 10, along with the full widths at the base and the emitted fluxes integrated over the emission lines.

\subsection{Photometric characteristics of the survey}

We present in Figs. 3, 4 and 5 the histograms of the $U$, $B$ magnitudes and of the $U-B$ indices for the different subsets of candidates.

On the basis of the $U-B$ index (Fig. 5), it is clear that the photometric errors are quite large, as expected: the cut-off that should be located around $U-B=-0.4$ is 
Table 8. Identification table of the emission lines observed in the spectra of the different quasars. The position of the line and its standard deviation are given in $\AA$. The redshift derived from the line is mentioned underneath. The last column but one gives the mean redshift attributed to the object and the related standard deviation based on the line to line dispersion. The bold characters indicate the lines used to derive the redshift. Vertical lines indicate the observed part of the spectra in the reference frame of the quasar. The last column refers to additional remarks to be found below the table. The three parts of the table correspond to different redshift ranges: a) $z>1.5$, b) $1.5>z>0.5$, c) $0.5>z$

\begin{tabular}{|c|c|c|c|c|c|c|c|}
\hline & $\begin{array}{c}\operatorname{Ly} \alpha \\
1215.67 \\
\end{array}$ & $\begin{array}{c}\mathrm{N} V \\
1240.81 \\
\end{array}$ & $\begin{array}{c}\text { Si [V/O IV ] } \\
1401.62 \\
\end{array}$ & $\begin{array}{c}\text { C IV } \\
1549.48 \\
\end{array}$ & $\begin{array}{c}\text { C III ] } \\
1907.64 / 1910.11\end{array}$ & $\begin{array}{l}\mathrm{z} \\
\sigma_{z}\end{array}$ & Rem. \\
\hline Q0107+003 & $\begin{array}{c}3640.7 \pm 0.9 \\
1.9956 \mathrm{a}\end{array}$ & & $\begin{array}{c}4174.7 \pm 1.9 \\
1.9793\end{array}$ & $\begin{array}{c}4575.3 \pm 9.2 \\
1.9536\end{array}$ & $\begin{array}{c}5622.7 \pm 7.7 \\
1.9445\end{array}$ & $\begin{array}{l}1.968 \\
0.023\end{array}$ & (1) \\
\hline Q0107-025C & & & & $\begin{array}{c}4461.1 \pm 1.3 \\
1.8799\end{array}$ & $\begin{array}{c}5550.2 \pm 1.3 \\
1.9065\end{array}$ & $\begin{array}{l}1.893 \\
0.019\end{array}$ & (2) \\
\hline Q0108+004 & $\begin{array}{c}3673.6 \pm 0.2 \\
2.0227\end{array}$ & & ID & $\begin{array}{c}4661.9 \pm 0.4 \\
2.0095\end{array}$ & $\underset{1.9963}{5721.6 \pm 1.3}$ & $\begin{array}{l}2.009 \\
0.013\end{array}$ & \\
\hline Q0109-014 & & & $\begin{array}{c}3837.6 \pm 1.0 \\
1.7387\end{array}$ & $\begin{array}{c}4265.3 \pm 0.7 \\
1.7535\end{array}$ & $\begin{array}{c}5237.4 \pm 0.5 \\
1.7463\end{array}$ & $\begin{array}{l}1.746 \\
0.007\end{array}$ & (3) \\
\hline Q0110-011 & $\begin{array}{c}3541.6 \pm 2.1 \\
1.9141 \mathrm{a}\end{array}$ & & $\begin{array}{c}4060.6 \pm 1.6 \\
1.8979:\end{array}$ & $\begin{array}{c}4466.9 \pm 1.7 \\
1.8837\end{array}$ & $\begin{array}{c}5498.2 \pm 0.7 \\
1.8793\end{array}$ & $\begin{array}{l}1.887 \\
0.009\end{array}$ & \\
\hline Q0111-005 & $\begin{array}{rl}3550.6 \pm & 9.0 \\
1.9215 & \mathrm{a}\end{array}$ & ID & $\begin{array}{c}4065.1 \pm 3.5 \\
1.9011\end{array}$ & $\begin{array}{c}4515.6 \pm 0.2 \\
1.9151\end{array}$ & & $\begin{array}{l}1.908 \\
0.010\end{array}$ & \\
\hline Q0112-012 & & & & $\begin{array}{c}4005.0 \pm 1.9 \\
1.5855\end{array}$ & $\begin{array}{c}4930.0 \pm 3.3 \\
1.5851\end{array}$ & $\begin{array}{l}1.585 \\
0.001\end{array}$ & (4) \\
\hline Q0113-010 & $\begin{array}{c}3629.2 \pm 2.8 \\
1.9862: a\end{array}$ & & ID & $\begin{array}{c}4597.9 \pm 3.0 \\
1.9682\end{array}$ & $\begin{array}{c}5614.5 \pm 1.9 \\
1.9402:\end{array}$ & 1.968 & \\
\hline Q0113-013 & $\begin{array}{c}3719.6 \pm 1.9 \\
2.0606 \mathrm{a}\end{array}$ & & $\underset{2.0555}{4281.4} \pm 1.7$ & $\begin{array}{c}4731.7 \pm 0.4 \\
2.0546\end{array}$ & & $\begin{array}{l}2.055 \\
0.001\end{array}$ & (5) \\
\hline Q0115-011 & $\begin{array}{rl}3884.4 \pm 2.0 \\
2.1962 & \mathrm{a}\end{array}$ & & $\begin{array}{c}4455.4 \pm 2.9 \\
2.1796\end{array}$ & $\begin{array}{c}4912.5 \pm 0.7 \\
2.1713\end{array}$ & $\begin{array}{c}6079.6 \pm 6.0 \\
2.1837:\end{array}$ & $\begin{array}{l}2.175 \\
0.006\end{array}$ & (6) \\
\hline Q0117-023 & $\begin{array}{c}3677.6 \pm 0.1 \\
2.0260\end{array}$ & $\begin{array}{c}3742.7 \\
2.0172\end{array}$ & $\underset{2.0082}{4215.1 \pm 0.7}$ & $\begin{array}{c}4680.2 \pm 0.3 \\
2.0214\end{array}$ & $\begin{array}{c}5771.0: \\
2.0221\end{array}$ & $\begin{array}{l}2.019 \\
0.007\end{array}$ & (7) \\
\hline Q0118-018 & $\begin{array}{rl}3588.2 \pm & 1.9 \\
1.9524 & \mathrm{a}\end{array}$ & & $\begin{array}{c}4091::: \\
1.9196 \quad:::\end{array}$ & $\begin{array}{c}4508.0 \pm 0.9 \\
1.9102\end{array}$ & $\begin{array}{c}5561.1 \pm 3.1 \\
1.9122\end{array}$ & $\begin{array}{l}1.911 \\
0.001\end{array}$ & \\
\hline Q0118-031B & & & & $\begin{array}{c}4821.7 \pm 1.3 \\
2.1127\end{array}$ & $\begin{array}{c}5918.3 \pm 1.3 \\
2.0993\end{array}$ & $\begin{array}{l}2.106 \\
0.009\end{array}$ & $\begin{array}{l}(8) \\
(27)\end{array}$ \\
\hline Q0119-009 & $\begin{array}{c}3601.4 \pm 0.2 \\
1.9633 \quad \mathrm{a}\end{array}$ & & $\begin{array}{c}4121.0 \pm 1.8 \\
1.9410\end{array}$ & $\begin{array}{c}4561.3 \pm 0.9 \\
1.9446\end{array}$ & $\begin{array}{c}5585.1 \pm 3.0 \\
1.9248\end{array}$ & $\begin{array}{l}1.943 \\
0.016\end{array}$ & (9) \\
\hline Q0121-008 & $\begin{array}{rl}3979.5 \pm 1.1 \\
2.2744 & \mathrm{a}\end{array}$ & & $\begin{array}{c}4535 . \pm 10 \\
2.2366:\end{array}$ & $\begin{array}{c}5038.0 \pm 0.7 \\
2.2523\end{array}$ & $2.22 \quad \cdots$ & 2.252 & (10) \\
\hline Q0122-028 & $\begin{array}{c}3682.8 \pm 0.2 \\
2.0302\end{array}$ & $\begin{array}{c}3746.8 \pm 0.4 \\
2.0205\end{array}$ & $\begin{array}{c}4239.7 \pm 0.1 \\
2.0257\end{array}$ & $\begin{array}{c}4673.2 \pm 1.3 \\
2.0168\end{array}$ & $\begin{array}{c}5758.3 \pm 1.9 \\
2.0155\end{array}$ & $\begin{array}{l}2.022 \\
0.006\end{array}$ & (5) \\
\hline Q0123-005A & ID & ID & & $\begin{array}{c}4474.9 \pm 1.3 \\
1.8888\end{array}$ & $\begin{array}{l}5525.0 \\
1.8933\end{array}::$ & 1.889 & \\
\hline Q0123-005B & & & ID & $\begin{array}{c}4282.8 \pm 0.9 \\
1.7648\end{array}$ & $\begin{array}{c}5265.6 \pm 1.4 \\
1.7610\end{array}$ & $\begin{array}{l}1.763 \\
0.003\end{array}$ & (11) \\
\hline Q0123-021 & $\begin{array}{rl}3566.5 \pm 0.1 \\
1.9346 & \mathrm{a}\end{array}$ & & $\begin{array}{c}4108.7 \pm 3.3 \\
1.9322\end{array}$ & $\begin{array}{c}4518.1 \pm 0.1 \\
1.9167\end{array}$ & $\begin{array}{c}5558.3 \pm 0.3 \\
1.9108\end{array}$ & $\begin{array}{l}1.914 \\
0.004\end{array}$ & (12) \\
\hline
\end{tabular}


Table 8. continued

\begin{tabular}{|c|c|c|c|c|c|c|c|c|}
\hline & $\begin{array}{c}\text { C IV } \\
1549.48 \\
\end{array}$ & $\begin{array}{l}\mathrm{He} \mathrm{II} \\
1640.4\end{array}$ & $\begin{array}{c}\text { O III ] } \\
1664 .\end{array}$ & $\begin{array}{c}\text { Al III } \\
1858.75 \\
\end{array}$ & $\begin{array}{l}\text { C III ] } \\
1907.64 \\
\end{array}$ & $\begin{array}{c}\mathrm{Mg} \mathrm{II} \\
2799.12 \\
\end{array}$ & $\begin{array}{c}z \\
\sigma_{z} \\
\end{array}$ & Rem. \\
\hline Q0107-025A & & & & & ID & $\begin{array}{c}5487.4 \pm 1.3 \\
0.9604\end{array}$ & $\begin{array}{l}0.960 \\
0.001\end{array}$ & (26) \\
\hline Q0107-025D & & & & & & $\begin{array}{c}4832.5 \\
0.7264\end{array}$ & $\begin{array}{l}0.726 \\
:\end{array}$ & (13) \\
\hline Q0107-025B & & & & & ID & $\begin{array}{c}5476.2 \pm 1.3 \\
0.9564\end{array}$ & $\begin{array}{l}0.956 \\
0.001\end{array}$ & (26) \\
\hline Q0108-007 & $\begin{array}{c}3754.9 \pm 1.4 \\
1.4240\end{array}$ & & & & $\underset{1.4252}{4625.1 \pm 1.0}$ & & $\begin{array}{l}1.424 \\
0.001\end{array}$ & (14) \\
\hline Q0108-020 & $\begin{array}{c}3566.6 \pm 0.6 \\
1.3025\end{array}$ & $\begin{array}{l}\text { 3773. : } \\
1.3007:\end{array}$ & $\begin{array}{l}\text { 3833. : } \\
1.3042:\end{array}$ & $\begin{array}{c}4275.3 \pm 3.8 \\
\quad 1.3008\end{array}$ & $\begin{array}{c}4391.1 \pm 0.2 \\
\quad 1.3025\end{array}$ & $\begin{array}{c}6439.3 \pm 0.3 \\
1.3004\end{array}$ & $\begin{array}{l}1.302 \\
0.001\end{array}$ & \\
\hline Q0108+001 & & & & & $\begin{array}{c}3763 . \pm 11 \\
0.9733 \mathrm{~b}\end{array}$ & $\begin{array}{c}5606.1 \pm 0.3 \\
\quad 1.0028\end{array}$ & 1.003 & (15) \\
\hline Q0110-019 & & & & & $\begin{array}{c}3995.8 \pm 1.2 \\
1.0952\end{array}$ & $\begin{array}{c}5884.3 \pm 0.8 \\
\quad 1.1021\end{array}$ & $\begin{array}{l}1.099 \\
0.005\end{array}$ & (16) \\
\hline Q0110-002B & & & & & $\begin{array}{c}3744.3 \pm 4.4 \\
0.9634\end{array}$ & $\begin{array}{c}5521.9 \pm 5.3 \\
0.9727\end{array}$ & $\begin{array}{l}0.968 \\
0.007\end{array}$ & \\
\hline Q0110-030 & $\begin{array}{c}3514.8 \pm 8.5 \\
1.2690:\end{array}$ & $\begin{array}{c}3717.6 \pm 4.4 \\
1.2669: P\end{array}$ & & & $\begin{array}{c}4275.5 \pm 4.2 \\
\quad 1.2419\end{array}$ & $\begin{array}{c}6239.6 \pm 3.3 \\
\quad 1.2291\end{array}$ & $\begin{array}{l}1.235 \\
0.009\end{array}$ & \\
\hline Q0110-006 & & & & & $\mathbf{P}$ & $\begin{array}{c}5415.3 \pm 1.6 \\
0.9346\end{array}$ & $\begin{array}{l}0.935 \\
:\end{array}$ & \\
\hline Q0111-007 & & & & & $\begin{array}{c}3797.5 \pm 3.1 \\
0.9912\end{array}$ & $\begin{array}{c}5597.4 \pm 2.6 \\
0.9997\end{array}$ & $\begin{array}{l}0.995 \\
0.006\end{array}$ & \\
\hline Q0113+000 & $\begin{array}{c}3533.7 \pm 0.6 \\
1.2812\end{array}$ & $\begin{aligned} 3731 . & : \\
1.2751 & ::\end{aligned}$ & & & $\begin{array}{c}4344.6 \pm 0.6 \\
1.2781\end{array}$ & $\begin{array}{c}6368.2 \pm 3.9 \\
1.2750:\end{array}$ & $\begin{array}{l}1.279 \\
0.002\end{array}$ & \\
\hline Q0113-009 & & & & & $\begin{array}{c}4316.1 \pm 3.4 \\
1.2631\end{array}$ & $\mathbf{P}$ & $\begin{array}{l}1.263 \\
:\end{array}$ & (17) \\
\hline Q0114-001 & $\begin{array}{c}3588.0 \pm 0.1 \\
1.3163\end{array}$ & $\begin{array}{c}3787 .:: \\
1.3093 \quad::\end{array}$ & $\begin{array}{l}3843 .:: \\
1.3102::\end{array}$ & $\begin{array}{l}4291 .: \\
1.3093::\end{array}$ & $\begin{array}{c}4416.8 \pm 0.4 \\
1.3160\end{array}$ & $\begin{array}{l}6467.8: \\
1.3106:\end{array}$ & $\begin{array}{l}1.316 \\
0.001\end{array}$ & \\
\hline Q0116-010 & & & & $\begin{array}{c}3812.5 \pm 0.8 \\
\quad 1.0517\end{array}$ & $\begin{array}{c}3906.2 \pm 1.2 \\
1.0482\end{array}$ & $\begin{array}{c}5754.7 \pm 6.3 \\
1.0558\end{array}$ & $\begin{array}{l}1.052 \\
0.004\end{array}$ & (18) \\
\hline Q0117+001 & & & & & & $\begin{array}{c}4614.3 \pm 0.2 \\
\quad 0.6485\end{array}$ & $\begin{array}{l}0.649 \\
:\end{array}$ & \\
\hline Q0117-024 & & & & & $\begin{array}{c}3741.3 \pm 0.6 \\
0.9618\end{array}$ & $\begin{array}{c}5483.8 \pm 1.2 \\
0.9591\end{array}$ & $\begin{array}{l}0.960 \\
0.002\end{array}$ & (19) \\
\hline Q0118-031A & $\begin{array}{c}3792.7 \pm 1.3 \\
1.4484\end{array}$ & & & & $\begin{array}{c}4673.7 \pm 1.3 \\
1.4507\end{array}$ & $\begin{array}{l}6917.0: \\
1.4711:\end{array}$ & $\begin{array}{l}1.450 \\
0.002\end{array}$ & $\begin{array}{l}\text { (20) } \\
\text { (27) }\end{array}$ \\
\hline Q0118-031C & & & & & $\begin{array}{c}4142.5 \pm 1.3 \\
\quad 1.1721\end{array}$ & $\begin{array}{c}6072.5 \pm 1.3 \\
1.1694\end{array}$ & $\begin{array}{l}1.171 \\
0.002\end{array}$ & (27) \\
\hline Q0120-001 & & & & & $\begin{array}{c}3643.3 \pm 1.1 \\
0.9104\end{array}$ & $\begin{array}{c}5341.4 \pm 1.0 \\
0.9082\end{array}$ & $\begin{array}{l}0.909 \\
0.001\end{array}$ & \\
\hline Q0120-029A & $\begin{array}{c}3882.4 \pm 7.0 \\
?\end{array}$ & & & & & $\begin{array}{c}5803.2 \pm 0.7 \\
1.0732 ? ?\end{array}$ & $\begin{array}{c}1.073 \\
?\end{array}$ & (28) \\
\hline Q0120-002 & $\begin{array}{c}3654.0 \pm 0.5 \\
1.3589\end{array}$ & $\mathbf{P}$ & & & $\begin{array}{c}4483.5 \pm 1.9 \\
1.3509\end{array}$ & & $\begin{array}{l}1.355 \\
0.006\end{array}$ & \\
\hline Q0120+002 & & & & & & $\begin{array}{c}4961.1 \pm 0.2 \\
0.7723\end{array}$ & 0.772 & (4) \\
\hline Q0121-022 & & & & & $\begin{array}{c}3784.6 \pm 2.7 \\
0.9845\end{array}$ & $\begin{array}{c}5572.9 \pm 3.1 \\
0.9909\end{array}$ & $\begin{array}{l}0.988 \\
0.005\end{array}$ & (21) \\
\hline
\end{tabular}


Table 8. continued

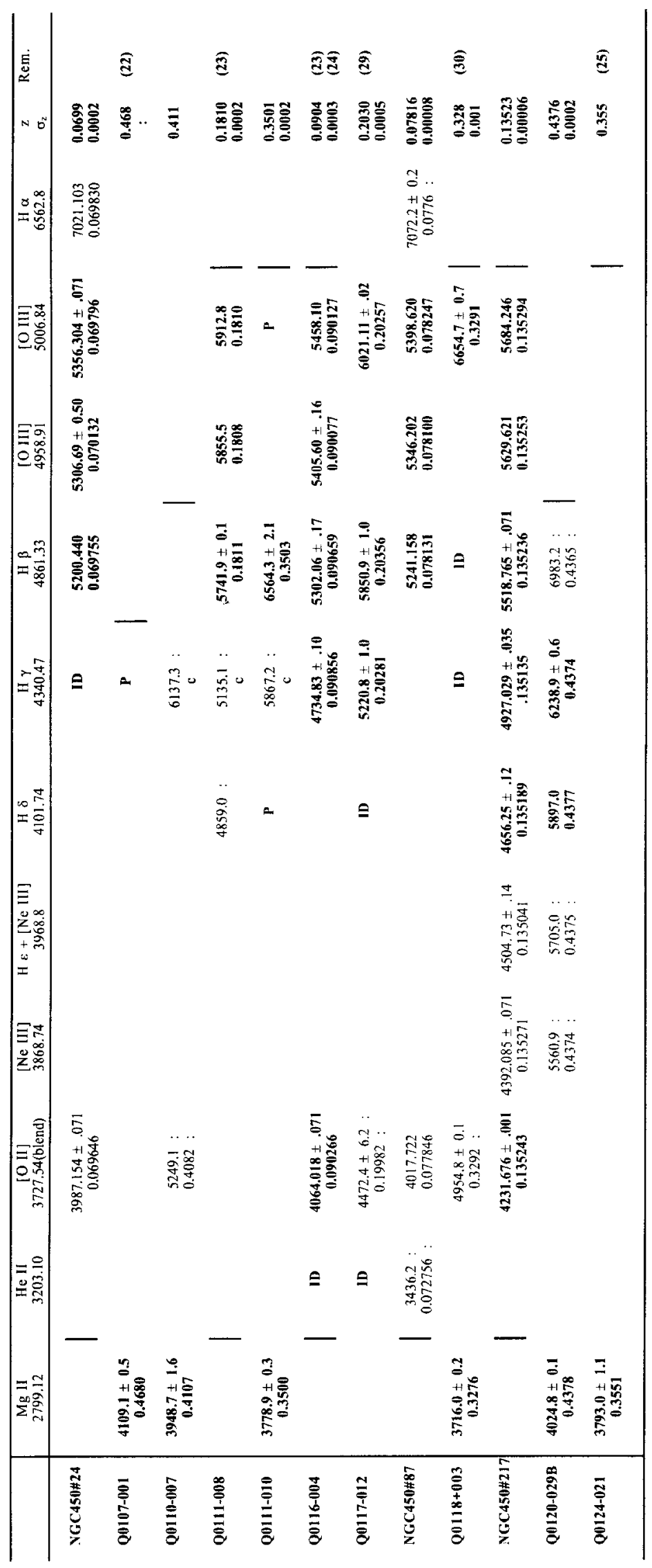


1 Noisy spectrum, possible absorption at $\lambda 6300 \AA$.

2 He II line at $\lambda 1640.4 \AA$ present.

${ }^{3}$ BAL quasar; P Cygni profile for $\mathrm{C}$ IV and possibly for Si IV/O IV]; the absorption could be double or multiple. $\mathrm{N}$ V is visible at the blue end of the spectrum.

4 Very noisy spectrum.

5 O I $\lambda 1304.4 \AA$ is possibly present.

${ }^{6}$ O I $\lambda 1304.4 \AA$, He II $\lambda 1640.4 \AA$ and N III] $\lambda 1750 \AA$ are possibly present.

$7 \mathrm{C}$ II $\lambda 1335.3 \AA$ is present; O I $\lambda 1304.4 \AA$ is possibly present.

${ }^{8} \mathrm{Ly} \alpha / \mathrm{N} \mathrm{V}$ is visible at the blue end of the spectrum; the blend Si IV/O IV] is also probably present; an absorption is clearly seen in the blue wing of $\mathrm{C}$ IV, other absorptions are possibly present around $\lambda 4070 \AA$.

9 Doublet of absorption lines most probable at $\lambda \lambda 3802-3820 \AA$; perhaps a few absorption lines in Ly $\alpha$.

10 Absorption system possibly present at $z=2.15$; partial absorption at $\lambda \lambda 3787,4155$ and $4883 \AA$.

11 Absorption structure present in the $\mathrm{C}$ IV line.

12 He II $\lambda 1640.4 \AA$, O III] $\lambda 1664 \AA$ and $\mathrm{Al}$ III $\lambda 1859 \AA$ are possibly present.

13 Several absorption lines potentially present.

14 Absorptions in the C IV and C III] lines; the redshifts are, at the level of our accuracy, identical $\left(z_{\mathrm{a}}=1.413\right)$.

15 Fe II blends possibly present.

16 Broad emission lines present at $\lambda 4348.9 \AA, \lambda 4883.0 \AA$ and $\lambda 5093.0 \AA$ (respectively $\lambda 2072.2 \AA, \lambda 2326.7 \AA$ and $\lambda 2426.7 \AA$ in the reference frame of the quasar); some of them are attributable to Fe II.

17 Sinewave type continuum (Fe II lines ?); probable absorptions.

18 Fe II strongly present.

19 Absorption components possibly present at $\lambda 3847.7 \AA$ and $\lambda 3913.7 \AA$ (and less certain at $\lambda 5639.0 \AA, \lambda 5687.0 \AA$ and $\lambda 5705.0 \AA$ ); Fe II present ?

20 An absorption component is possibly present at $\lambda 4075 \AA$.

21 Probable absorption around $\lambda 4781 \AA$ and perhaps also around $\lambda 6137 \AA$.

22 Absorption in the blue wing of the line; $z_{\mathrm{a}}=0.448$ : (if $\mathrm{Mg}$ II is the correct identification).

$23[\mathrm{Ne} \mathrm{v}] \lambda 3425.8 \AA$ may be present.

24 A possible slight difference in redshift between permitted and forbidden emission lines.

25 Many Fe II lines.

${ }^{26}$ See Surdej et al. (1986); they also performed another determination of $z$ based on higher-dispersion data.

27 See Robertson et al. (1986); they also performed another determination of $z$ based on higher-dispersion data.

28 Strangely shaped emission line at $\lambda 6410.1 \AA$.

29 Broad line at $\lambda 6685 \AA$ or artefact?

30 Probable absorption around $\lambda 5121 \AA$.

: For definitive redshifts, the colon indicates a remaining doubt because the value is based on the identification of only one line; in other circumstances, an uncertain value.

:: Very uncertain value.

::: Extremely uncertain value.

? Very uncertain redshift due to a problem in the line identification.

a Contaminated by $\mathrm{N}$ v.

b Contaminated by $\mathrm{Al}$ III.

c Contaminated by [O III] $\lambda 4363.21 \AA$.

ID Present but ill-defined.

P Possibly present.

far from being total and several objects are redder than $U-B=-0.2$. The cut-off is nevertheless visible around $U-B=-0.3$ for the primary candidates. The exact position of this cut-off is very important to fix the characteristics of the survey; we thus attempted to refine the abovementioned values. We generated populations of objects with tail-like distributions of $U-B$ (exponential or linear probability density function, pdf) and a perfect cut-off. We then added to each object a random error drawn out from a gaussian distribution with zero mean and $\sigma=0.15$. For different positions of the cut-off, we computed the twosample Kolmogorov-Smirnov statistic which is a measure of the similarity between the generated population and our data. We thus obtain a mean value of the statistic as well as a dispersion over the simulations, all that as a function of the cut-off value. For the primary candidates, the mean Kolmogorov-Smirnov statistic exhibits a minimum for a cut-off of $U-B=-0.30 \pm 0.04$. The error represents one standard deviation of the dispersion around the minimum as deduced from the dispersion of the statistic. For the primary+secondary candidate dataset, we rather obtain $U-B=-0.20$ which indicates that at least some of the secondary candidates are less blue than the primary ones, as expected. An alternative approach consists 
Table 9. Total and equivalent widths of the emission lines observed in the spectra of the different quasars. The configuration of this table is as that of Table 8 . We give, on each first row, the total width at the bottom of the emission lines $(\AA)$ and on each second row, their equivalent width $(\AA)$ in the reference frame of the quasar. Again the three parts correspond to the different redshift ranges

\begin{tabular}{|c|c|c|c|c|c|c|}
\hline & $\begin{array}{c}\text { Ly } \alpha \\
1215.67 \mathrm{a} \\
\end{array}$ & $\begin{array}{c}\text { Si IV/O IV ] } \\
1401.62 \\
\end{array}$ & $\begin{array}{c}\text { C IV } \\
1549.48 \\
\end{array}$ & $\begin{array}{l}\mathrm{He} \text { II } \\
1640.4\end{array}$ & $\begin{array}{c}\text { C III ] } \\
1907.64 / 1910.11 \\
\end{array}$ & Rem. \\
\hline Q0107+003 & $\begin{array}{l}80.9 \pm 2.7 \\
49.5 \pm 1.7\end{array}$ & $\begin{array}{l}41.4 \pm 9.5 \\
10.7 \pm 0.3\end{array}$ & $\begin{array}{l}82.7 \pm 6.8 \\
25.7 \pm 0.4\end{array}$ & & $\begin{array}{r}110.6 \pm 5.4 \\
21.8 \pm 1.9\end{array}$ & \\
\hline Q0107-025C & & & $\begin{array}{l}96.7 \pm 2.4 \\
43.6 \pm 2.2\end{array}$ & $\begin{array}{r}40.0 \pm 2.8 \\
5.2 \pm 0.5:\end{array}$ & $\begin{array}{r}108.7 \pm 4.5 \\
18.0 \pm 0.2\end{array}$ & \\
\hline$Q 0108+004$ & $\begin{array}{l}81.6 \pm 12.0:: \\
74.6 \pm 19.4::\end{array}$ & & $\begin{array}{l}79.8 \pm 2.7 \\
36.0 \pm 1.5\end{array}$ & & $\begin{array}{l}47.0 \pm 8.1 \\
12.4 \pm 0.9\end{array}$ & \\
\hline Q0109-014 & & $\begin{array}{l}56.1 \pm 5.5 \\
10.8 \pm 0.5:\end{array}$ & $\begin{array}{l}63.6 \pm 0.8 \\
18.9 \pm 0.4:\end{array}$ & & $\begin{array}{l}134 . \pm 15 . \\
32.6 \pm 6.1\end{array}$ & \\
\hline Q0110-011 & $\begin{array}{l}59.6 \pm 1.2 \\
40.0 \pm 3.2:\end{array}$ & $\begin{array}{r}63.8 \pm 1.2 \\
8.6 \pm 1.0:\end{array}$ & $\begin{array}{r}102.6 \pm 4.8 \\
27.1 \pm 0.5\end{array}$ & & $\begin{array}{r}122.0 \pm 2.4 \\
26.5 \pm 1.5:\end{array}$ & \\
\hline Q0111-005 & & $\begin{array}{l}49.6 \pm 6.0 \\
22.0 \pm 2.2\end{array}$ & $\begin{array}{r}59 . \pm 13 . \\
26.6 \pm 1.9\end{array}$ & & & \\
\hline Q0112-012 & & & $\begin{array}{r}113.0 \pm 7.1 \\
25.5 \pm 1.6\end{array}$ & & $\begin{array}{l}\text { 149. } \pm 13 \\
44 . \pm 11 .\end{array}$ & \\
\hline Q0113-010 & $\begin{array}{l}76.2 \pm 1.2 \\
76.5 \pm 10.6\end{array}$ & & $\begin{array}{l}97.1 \pm 8.8 \\
21.6 \pm 3.8:\end{array}$ & & $\begin{array}{r}103.8 \pm 4.2 \\
42.6 \pm 2.3:\end{array}$ & \\
\hline Q0113-013 & $\begin{array}{r}103.5 \pm 4.1 \\
91.2 \pm 4.1\end{array}$ & $\begin{array}{l}73.4 \pm 6.9 \\
21.2 \pm 3.5\end{array}$ & $\begin{array}{l}81.2 \pm 4.5 \\
40.4 \pm 2.5\end{array}$ & & & \\
\hline Q0115-011 & $\begin{array}{l}82.4 \pm 8.9 \\
60.2 \pm 4.2\end{array}$ & $\begin{array}{l}55 . \pm 13 . \\
8.5 \pm 0.8\end{array}$ & $\begin{array}{l}65.6 \pm 2.5 \\
24.6 \pm 1.9\end{array}$ & & $\begin{array}{r}52 . \pm 16 .: \\
15.0 \pm 3.0:\end{array}$ & \\
\hline Q0117-023 & $\begin{array}{l}\text { 85. } \pm 17 . \\
207 . \pm 39 .:\end{array}$ & $\begin{array}{l}50.4 \pm 1.1 \\
24.5 \pm 3.4:\end{array}$ & $\begin{array}{l}79.5 \pm 5.3 \\
69.7 \pm 4.8\end{array}$ & & & \\
\hline Q0118-018 & $\begin{array}{l}81.6 \pm 8.4 \\
72.7 \pm 3.5:\end{array}$ & $\begin{array}{l}70.6 \pm 6.9 \\
14.9 \pm 3.0\end{array}$ & $\begin{array}{r}66 . \pm 13 \\
25.4 \pm 5.0\end{array}$ & & $\begin{aligned} & 81 . \pm 11 \\
& 26.7 \pm 3.8\end{aligned}$ & \\
\hline Q0118-031B & & & $\begin{array}{l}61.6 \pm 0.3 \\
25.3 \pm 1.0\end{array}$ & & $\begin{array}{l}\text { 114. } \pm 14 .: \\
32.0 \pm 4.9:\end{array}$ & (1) \\
\hline Q0119-009 & $\begin{array}{l}104 . \pm 10 . \\
88.1 \pm 9.8\end{array}$ & $\begin{array}{l}54.4 \pm 8.2 \\
12.9 \pm 1.6\end{array}$ & $\begin{array}{l}70.7 \pm 7.2 \\
16.4 \pm 3.1\end{array}$ & & $\begin{array}{r}63 . \pm 15 \\
13.8 \pm 2.4\end{array}$ & \\
\hline Q0121-008 & $\begin{array}{l}84.5 \pm 1.4 \\
51.4 \pm 2.4\end{array}$ & $\begin{array}{r}59.1 \pm 2.5: \\
8.6 \pm 1.1:\end{array}$ & $\begin{aligned} 94 . & \pm 18 . \\
21.5 & \pm 5.1\end{aligned}$ & & $\begin{array}{l}92.7 \pm 7.9: \\
26.1 \pm 2.3:\end{array}$ & \\
\hline Q0122-028 & $\begin{array}{l}59.2 \pm 6.7 \\
60.2 \pm 9.3\end{array}$ & $\begin{array}{l}30 . \pm 11 .:: \\
7.6 \pm 1.3::\end{array}$ & $\begin{array}{r}49 . \pm 11 \\
21.1 \pm 2.5\end{array}$ & & $\begin{array}{r}59 . \pm 14 . \\
28.9 \pm 4.0\end{array}$ & \\
\hline Q0123-005A & & & $\begin{array}{l}121 . \pm 15 \\
72.2 \pm 3.6\end{array}$ & & & \\
\hline Q0123-005B & & & $\begin{array}{l}99.2 \pm 2.5 \\
36.6 \pm 2.0:\end{array}$ & & $\begin{array}{r}125.3 \pm 3.3 \\
39.8 \pm 3.4\end{array}$ & \\
\hline Q0123-021 & $\begin{array}{l}65.9 \pm 2.7: \\
48.8 \pm 2.2:\end{array}$ & $\begin{array}{l}73.2 \pm 3.2 \\
11.8 \pm 0.2\end{array}$ & $\begin{array}{c}98.9 \\
35.1 \pm 4.6\end{array}$ & & $\begin{array}{l}84.2 \pm 8.4 \\
28.6 \pm 1.2\end{array}$ & \\
\hline
\end{tabular}


Table 9. continued

\begin{tabular}{|c|c|c|c|c|c|c|c|}
\hline & $\begin{array}{c}\text { C IV } \\
1549.48 \\
\end{array}$ & $\begin{array}{c}\text { He II } \\
1640.4\end{array}$ & $\begin{array}{c}\text { O III ] } \\
\text { [664. }\end{array}$ & $\begin{array}{c}\text { Al III } \\
1858.75 \\
\end{array}$ & $\begin{array}{l}\text { C III ] } \\
1907.64 \\
\end{array}$ & $\begin{array}{c}\mathrm{Mg} \mathrm{II} \\
2799.12 \\
\end{array}$ & Rem. \\
\hline Q0107-025A & & & & & & $\begin{array}{r}205.3 \pm 7.7 \\
89.1 \pm 6.2\end{array}$ & (2) \\
\hline Q0107-025D & & & & & & $\begin{array}{l}187 . \pm 28 . \\
25.0 \pm 2.7\end{array}$ & \\
\hline Q0107-025B & & & & & & $\begin{array}{r}210.1 \pm 4.1 \\
45.4 \pm 2.5\end{array}$ & (2) \\
\hline Q0108-007 & $\begin{array}{r}95.8 \pm 5.2 \\
108.2 \pm 9.3\end{array}$ & & & & $\begin{array}{l}91.6 \pm 0.1 \\
36.2 \pm 1.6:\end{array}$ & & \\
\hline Q0108-020 & $\begin{array}{l}55.6 \pm 2.7 \\
130 . \pm 29 .\end{array}$ & $\begin{array}{c}17.0 \\
12.7 \pm 1.4\end{array}$ & $\begin{array}{l}20.4 \pm \mathbf{3 . 8} \\
13.0 \pm \mathbf{2 . 8}\end{array}$ & $\begin{array}{l}30.9 \pm 6.2: \\
13.1 \pm 0.7:\end{array}$ & $\begin{array}{l}57.4 \pm 7.9 \\
51.8 \pm 9.0\end{array}$ & $\begin{array}{l}62.6 \pm 3.5 \\
65.2 \pm 3.5\end{array}$ & \\
\hline Q0108+001 & & & & & $\begin{array}{r}117.2 \pm 2.3 \quad b \\
34.9 \pm 0.4\end{array}$ & $\begin{aligned} 76 . & \pm 11 . \\
13.7 \pm 1.3 & \end{aligned}$ & \\
\hline Q0110-019 & & & & & $\begin{array}{l}92 . \pm 13 . \\
7.4 \pm 0.4:\end{array}$ & $\begin{array}{r}152.5 \pm 7.4 \\
27.8 \pm 1.9\end{array}$ & \\
\hline Q0110-002B & & & & & $\begin{aligned} & 175.9 \pm 7.7 \\
& 67 . \pm 11 .:\end{aligned}$ & $\begin{array}{r}192.2 \pm 8.1 \\
34.6 \pm 0.9:\end{array}$ & \\
\hline Q0110-030 & $\begin{array}{l}50.1 \pm 3.6: \\
11.2 \pm 1.8:\end{array}$ & $\begin{array}{r}34.6 \pm 2.1: \\
3.7 \pm 0.4:\end{array}$ & & & $\begin{array}{l}152 . \pm 14 . \\
24.2 \pm 1.0\end{array}$ & $\begin{array}{r}132.5 \pm 9.5 \\
25.1 \pm 1.0\end{array}$ & \\
\hline Q0110-006 & & & & & & $\begin{array}{r}247 . \pm 28 . \\
106.0 \pm 7.8:\end{array}$ & \\
\hline Q0111-007 & & & & & $\begin{array}{l}112 . \pm 15 .: \\
33.4 \pm 0.1:\end{array}$ & $\begin{array}{l}180 . \pm 14 .: \\
49.6 \pm 2.5:\end{array}$ & \\
\hline Q0113+000 & $\begin{array}{r}68.5 \pm 2.0: \\
94 . \pm 11 .:\end{array}$ & & & & $\begin{array}{r}84 . \pm 14 \\
33.6 \pm 2.0\end{array}$ & $\begin{array}{l}\text { 126. } \pm 16 .:: \\
76 . \pm 20 .:\end{array}$ & \\
\hline Q01 13-009 & & & & & $\begin{array}{l}213 . \pm 22 . \\
43.4 \pm 2.0:\end{array}$ & & \\
\hline Q01 14-001 & $\begin{array}{l}50.7 \pm 4.0 \\
35.7 \pm 1.3\end{array}$ & & & & $\begin{array}{l}65.7 \pm 6.7 \\
24.9 \pm 3.6\end{array}$ & $\begin{array}{r}81 . \pm 14 . \\
27.8 \pm 4.7\end{array}$ & \\
\hline Q0116-010 & & & & $\begin{array}{l}120 . \\
22.5\end{array}$ & $\begin{array}{l}13 . \\
2.3\end{array}$ & $\begin{array}{r}150.2 \pm 2.8:: \\
21.2 \pm 3.9:\end{array}$ & \\
\hline Q0117+001 & & & & & & $\begin{array}{l}118 . \pm 47 . \\
24.5 \pm 2.8\end{array}$ & \\
\hline Q0117-024 & & & & & $\begin{array}{l}\mathbf{8 8 . 8} \pm \mathbf{3 . 1} \\
\mathbf{2 6 . 8} \pm \mathbf{0 . 8}:\end{array}$ & $\begin{array}{l}151 . \pm 20 . \\
37.5 \pm 4.8\end{array}$ & \\
\hline Q0118-031A & $\begin{array}{r}118.0 \pm 2.5 \\
29.0 \pm 4.8\end{array}$ & & & & $\begin{array}{r}112.0 \pm 3.4 \\
24.2 \pm 2.0\end{array}$ & $\begin{array}{l}143 . \pm 11,: \\
30.4 \pm 2.3:\end{array}$ & (1) \\
\hline Q0118-031C & & & & & $\begin{array}{l}68.5 \pm 3.1 \\
44.5 \pm 2.4\end{array}$ & $\begin{array}{l}96.6 \pm 4.9 \\
42.2 \pm 3.2\end{array}$ & (1) \\
\hline Q0120-001 & & & & & $\begin{array}{l}134 . \pm 15 \\
44.6 \pm 3.3:\end{array}$ & $\begin{array}{l}106 . \pm 11 \\
23.2 \pm 1.0:\end{array}$ & \\
\hline Q0120-029A & $\begin{array}{l}162 . \pm 16 . \\
16.2 \pm 2.0 ?\end{array}$ & & & & & $\begin{array}{l}125 . \pm 19 \\
16.6 \pm 2.1 ?\end{array}$ & (3) \\
\hline Q0120-002 & $\begin{array}{l}94.3 \pm 9.2 \\
44.4 \pm 1.9:\end{array}$ & & & & $\begin{array}{r}128.5 \pm 5.3 \\
36.9 \pm 3.5\end{array}$ & & \\
\hline$Q 0120+002$ & & & & & & $\begin{array}{l}110 . \pm 23 \\
65.7 \pm 5.4\end{array}$ & \\
\hline Q0121-022 & & & & & $\begin{aligned} 85 . & \pm 26 . \\
29.0 & \pm 3.5\end{aligned}$ & $\begin{array}{l}\text { 128. } \pm 69 .: \\
23.9 \pm 6.0\end{array}$ & \\
\hline
\end{tabular}


Table 9. continued

\begin{tabular}{|c|c|c|c|c|c|}
\hline & $\begin{array}{c}\mathrm{Mg} \text { II } \\
2799.12\end{array}$ & $\begin{array}{c}\mathrm{H} \mathrm{\gamma} \\
4340.47 \\
\end{array}$ & $\begin{array}{c}\text { H } \beta \\
4861.33\end{array}$ & $\begin{array}{l}\text { [ O III ] } \\
4958.91\end{array}$ & 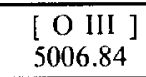 \\
\hline Q0107-001 & $\begin{array}{l}176 . \pm 13 \\
40.8 \pm 3.0\end{array}$ & & & & \\
\hline Q0110-007 & $\begin{array}{r}177.3 \pm 4.9 \\
29.3 \pm 3.6\end{array}$ & & & & \\
\hline Q0111-008 & & & $\begin{array}{l}73.4 \pm 4.0 \\
43.5 \pm 0.9\end{array}$ & $\begin{array}{r}17.2 \pm 1.3 \\
7.3 \pm 0.3\end{array}$ & $\begin{array}{l}32.8 \pm 2.6 \\
21.1 \pm 2.1\end{array}$ \\
\hline Q0111-010 & $\begin{array}{r}179.3 \pm 2.6 \\
49.3 \pm 6.0:\end{array}$ & & $\begin{array}{l}\text { 222. } \pm \text { 39. } \\
\text { 86. } \pm 21 .:\end{array}$ & & \\
\hline Q0117-012 & & $\begin{array}{l}77.6 \pm 3.8 \\
14.9 \pm 1.7\end{array}$ & $\begin{array}{l}51.0 \pm 3.8 \\
28.5 \pm 0.7\end{array}$ & & $\begin{array}{c}26.6 \\
5.4 \pm 0.3\end{array}$ \\
\hline Q0118+003 & $\begin{array}{r}142 . \pm 36 . \\
106.6 \pm 9.9\end{array}$ & & & & \\
\hline Q0120-029B & $\begin{array}{l}187 . \pm 16 . \\
32.5 \pm 2.3\end{array}$ & $\begin{array}{l}170 . \pm 17 \\
33.8 \pm 1.7\end{array}$ & & & \\
\hline Q0124-021 & $\begin{array}{l}176 . \pm 17 . \\
56.6 \pm 4.0\end{array}$ & & & & \\
\hline
\end{tabular}

1 See Robertson et al. (1986).

2 See Surdej et al. (1986).

3 A third line at $\lambda 6410.1 \AA$ has a total width of $91.7 \pm 6.0 \AA$ and an equivalent width of $9.3 \pm 1.2 \AA$.

: Uncertain value.

:: Very uncertain value.

? Very uncertain value due to a problem in the line identification.

a Contaminated by $\mathrm{N}$.

b Contaminated by $\mathrm{Al}$ III.

Table 10. Total widths and integrated fluxes of the emission lines observed in the spectra of objects with a faint continuum. The configuration and legend of this table are as those of Table 9. We give on each first row the total width at the bottom of the emission lines $(\AA)$ and, on the second row, the emitted flux integrated over the lines (arbitrary units)

\begin{tabular}{|c|c|c|c|c|c|c|}
\hline & $\begin{array}{c}{[\mathrm{O} \text { II }]} \\
3727.54 \text { (blend) } \\
\end{array}$ & $\begin{array}{l}{[\mathrm{Ne} \text { III] }} \\
3868.74\end{array}$ & $\begin{array}{c}\mathrm{H} \gamma \\
4340.47 \\
\end{array}$ & $\begin{array}{c}\mathrm{H} \beta \\
4861.33\end{array}$ & $\begin{array}{l}\text { [ O III ] } \\
4958.91\end{array}$ & $\begin{array}{l}{[\text { O III ] }} \\
5006.84\end{array}$ \\
\hline NGC450\#24 & $\begin{aligned} 14.3 & \pm 1.2 \\
43.03 & \pm 0.11\end{aligned}$ & & & $\begin{array}{r}9.82 \pm 0.47 \\
18.14 \pm 0.84\end{array}$ & $\begin{aligned} 10.29 & \pm 0.47 \\
5.78 & \pm 0.32\end{aligned}$ & $\begin{aligned} 13.6 & \pm 2.1 \\
15.44 & \pm 0.61\end{aligned}$ \\
\hline Q0116-004 & & & $\begin{array}{l}15.29 \pm 0.70 \\
17.38 \pm 0.83\end{array}$ & $\begin{aligned} 29.98 & \pm 0.27 \\
69.6 & \pm 2.4\end{aligned}$ & $\begin{array}{c}10.1 \pm 1.4 \\
12.21 \pm 0.10\end{array}$ & $\begin{array}{l}19.9 \pm 2.8 \\
50.4 \pm 2.5\end{array}$ \\
\hline NGC450\#87 & $\begin{array}{l}16.08 \pm 0.71 \\
28.50 \pm 0.60\end{array}$ & & & $\begin{array}{l}10.37 \pm 0.71 \\
10.01 \pm 0.27\end{array}$ & $\begin{array}{l}7.58 \pm 0.27 \\
8.67 \pm 0.12\end{array}$ & $\begin{array}{l}11.76 \pm 0.27 \\
23.29 \pm 0.32\end{array}$ \\
\hline NGC450\#217 & $\begin{array}{l}15.86 \pm \mathbf{0 . 7 6} \\
89.37 \pm \mathbf{0 . 0 6}\end{array}$ & $\begin{array}{l}13.37 \pm 0.92 \\
16.02 \pm 0.48\end{array}$ & $\begin{array}{l}10.43 \pm 0.92 \\
17.73 \pm \mathbf{0 . 4 4}\end{array}$ & $\begin{array}{l}12.93 \pm 0.26 \\
43.16 \pm 0.56\end{array}$ & $\begin{aligned} 14.3 & \pm 1.1 \\
50.94 & \pm 0.65\end{aligned}$ & $\begin{array}{l}16.89 \pm 0.26 \\
163.6 \pm 2.6\end{array}$ \\
\hline
\end{tabular}


(a) Primary candidates

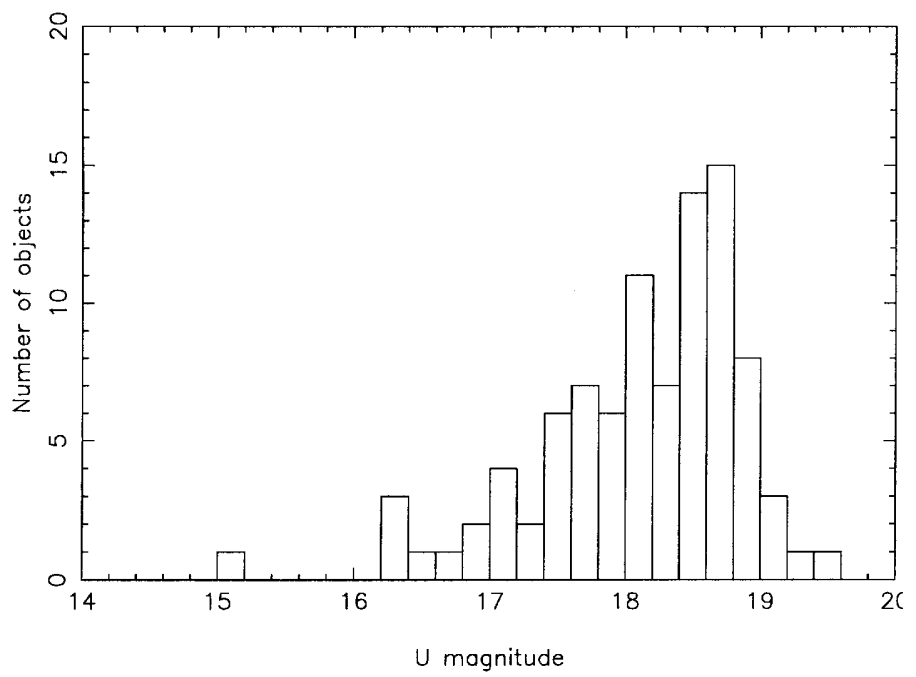

(b) Primary+secondary candidates

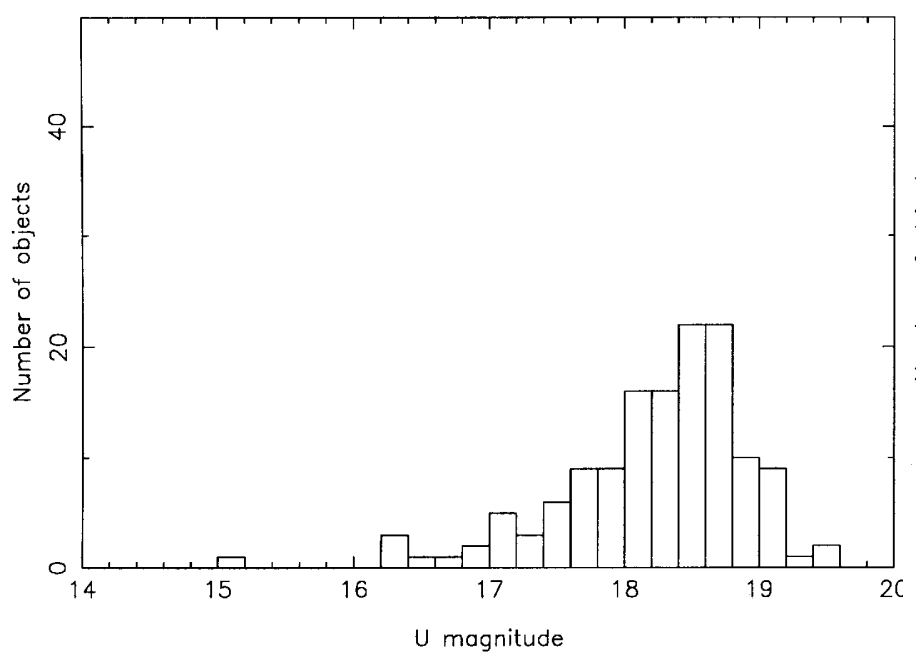

Fig. 3. $U$ magnitude histograms of the objects: a) primary candidates, b) primary + secondary candidates; one bin corresponds to $0.2 \mathrm{mag}$

in computing, from the calibrations, the $U-B$ colour index corresponding to strictly equal $\log$ (flux) in both images. Of course, this is not strictly similar to what we actually did because the $U$ image is slightly blurred compared to the $B$ one; it remains unclear to which aspect the eye is the most sensitive: to the size of the image or to the central density? Probably to a combination of both but this combination is not necessarily equivalent to the integrated density and may be variable with the brightness of the object. The $U-B$ colour index for equal $\log$ (flux) is clearly a function of the flux. For $B$ between 17 and 19, we have $U-B \simeq-0.3$ in good agreement with the above results. Outside that region, the $U-B$ is slightly bluer $(\simeq-0.4)$. (a) Primary candidates

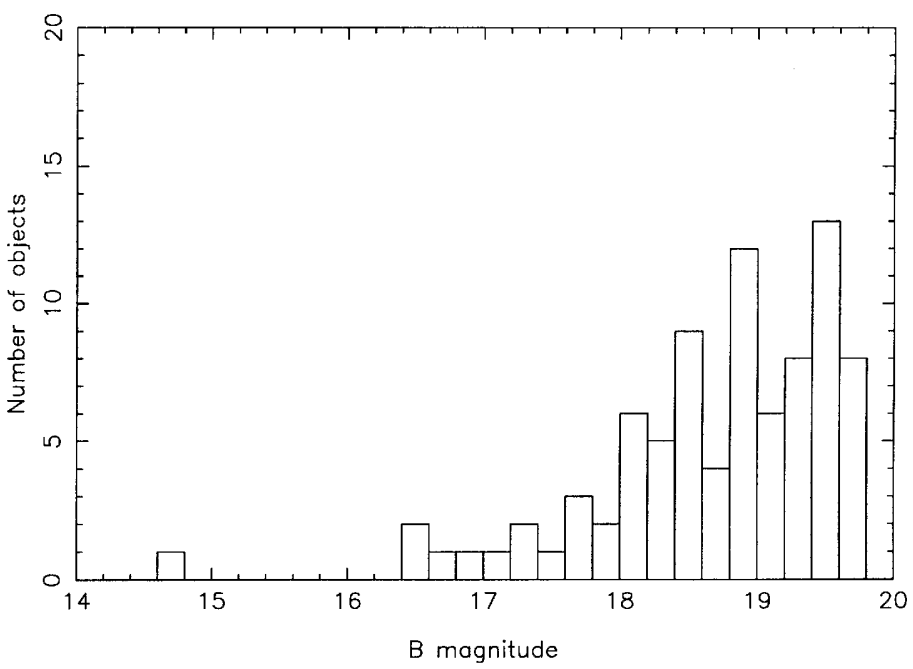

(b) Primary+secondary candidates

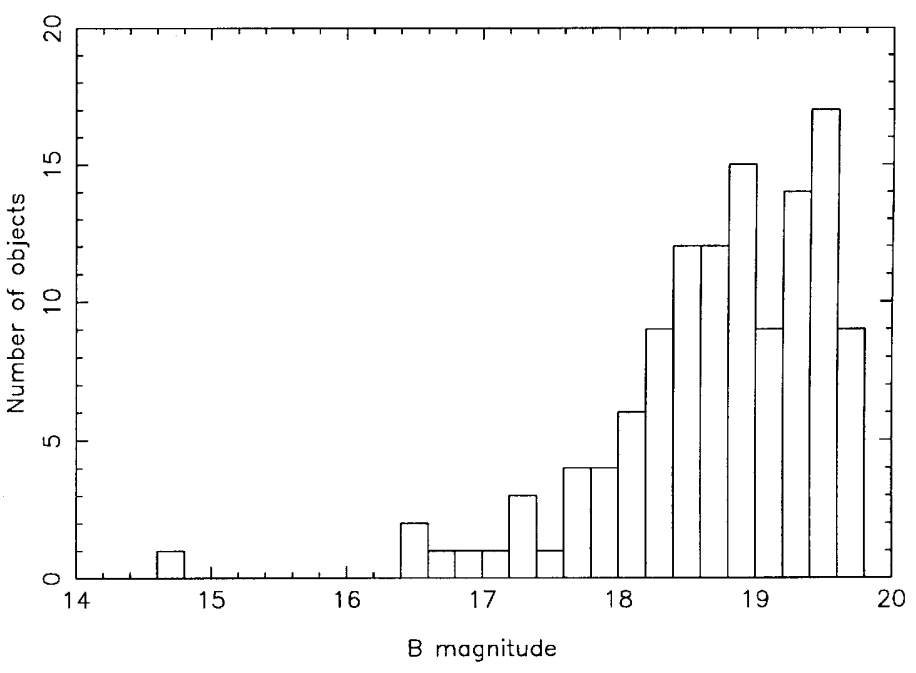

Fig. 4. $B$ magnitude histograms of the objects: a) primary candidates, b) primary + secondary candidates; one bin corresponds to $0.2 \mathrm{mag}$

Let us turn now to the histogram of the $B$ magnitudes. The main cut-off is at $B=19.6$ : beyond, there is a marked drop of the bin counts, indicating the limiting magnitude of the survey. Another - more surprising - result is the fact that another cut-off seems to be present at $B=19.0$. Beyond, there is a marked decrease of the bin counts where one expects a continuing increase. The origin of this effect is unknown; it could be either real or induced by an observational problem and deserves further attention. This could also be related to the above-mentioned possible dependence of the $U-B$ cut-off on the brightness of the object. Our on-going $U B V R I$ survey should shed some light on this problem. The histogram of the $U$ magnitudes exhibits another behaviour, with the existence of a cut-off at $U=18.8$, but with still large counts beyond. In 
(a) Quasars and related AGN

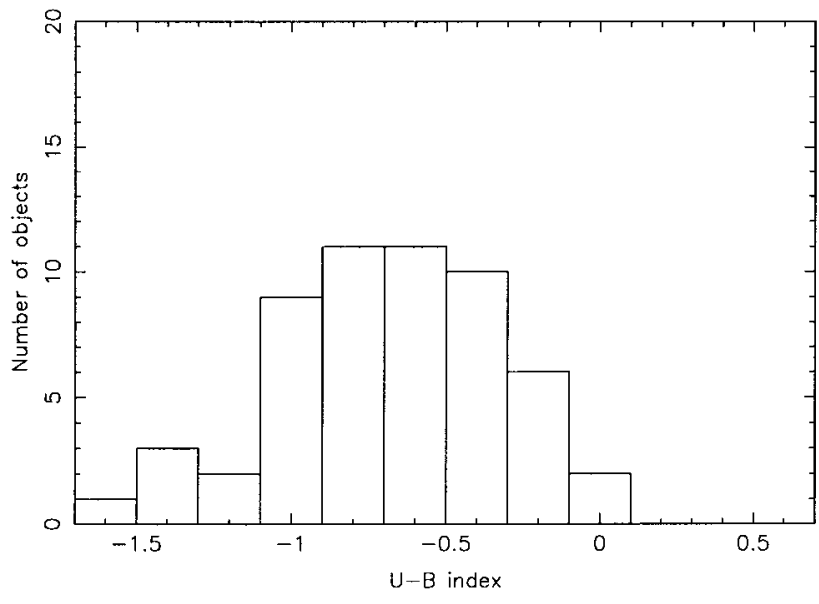

(b) Primary condidates

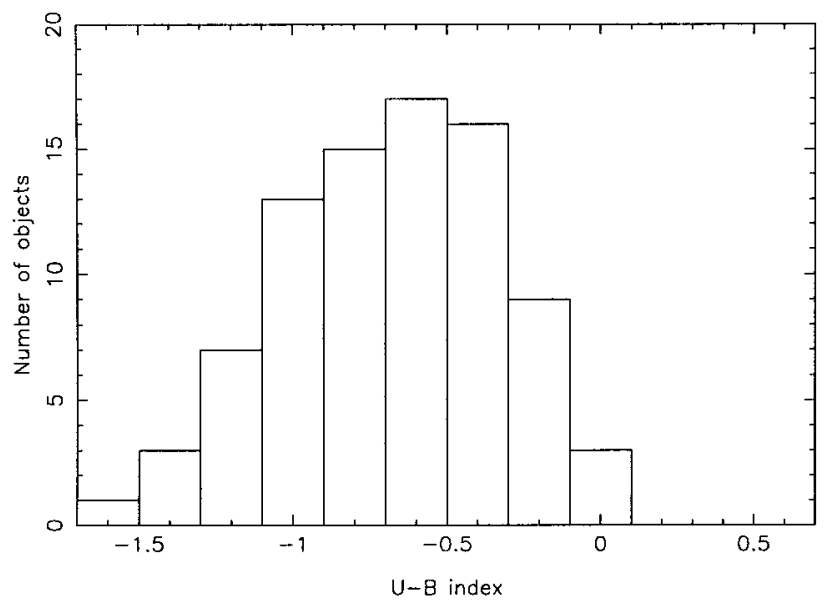

(c) Primary+secondary condidates

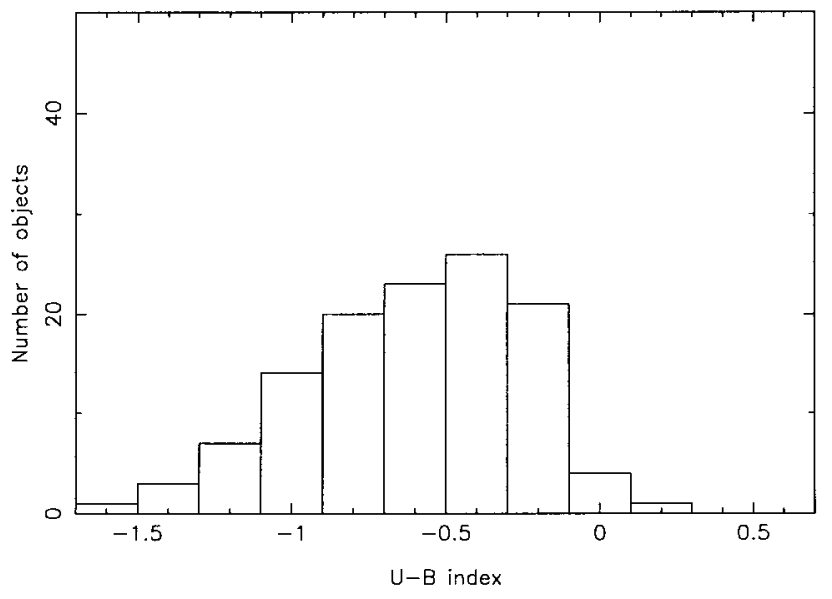

Fig. 5. $U-B$ histograms of the objects: a) quasars and related AGN, b) primary candidates, c) primary + secondary candidates; one bin corresponds to $0.2 \mathrm{mag}$ fact the counts at the faint end vanish progressively with no indication of a limiting magnitude. The explanation is that, as most of the blue candidates have $U-B$ indices between -0.3 and -0.8 , the $B$ magnitudes of the bluer objects having $U>18.8$ are fainter than the limiting $B$ magnitude of the survey. These objects are expected to be lost because for some of them only one image $(U)$ is present and it is mistakenly identified as an isolated $B$ image, typical of the very common red objects.

We compare our surface density of quasars with the results from other surveys in Table 11. We also give the power-law suggested by Braccesi et al. (1980; see also Boyle et al. 1987) which beyond $B=19.0$ should be considered as an extrapolation. For objects brighter than 18.5, our density compares well with those of other surveys. For the magnitude range $18.5-19.0$, our values are slightly lower but this can be partly explained by the fact that the secondary candidates are not yet identified; they are particularly numerous in this magnitude range. For the magnitude range 19.0 - 19.5, we have a very strong depletion of quasars indicating that we are far under completeness for this last bin (i.e. the nearest to the limiting magnitude).

\subsection{Cross-identification with other surveys}

Out of the 59 quasars originally present in the list of the primary candidates, 6 were already suspected to be quasars in the framework of the University of Michigan objective-prism survey (McAlpine \& Lewis 1978): 3 probable QSOs UM $314(z \simeq 2.15)$, UM $315(z \simeq 2.09)$, UM 322 $(z \simeq 1.99)$ and 3 possible ones UM $310(z \simeq 1.38)$, UM 316 (no $z$ ) and UM 324 (no $z$ ). McAlpine \& Feldman (1982) performed the spectroscopic identification and refined some redshifts: UM $314(z=2.19)$, UM $315(z=2.05)$, UM $316(z=0.96)$, UM $322(z=1.93)$ and UM 324 $(z=0.35)$. As for UM 310, it was identified with the Parkes radiosource PKS 0112 - 017 (Wall et al. 1971) for which Wills \& Lynds (1978) proposed a redshift $z=1.365$. We reobserved UM 310 (三 Q0112 - 017), UM 314 ( $\equiv \mathrm{Q} 0115$ - 011), UM 316 (三Q0117 - 024), UM 322 (三Q0123 - 021) and UM 324 (三Q Q0124-021) and improved the redshift value for the last four quasars. We adopted the published value for UM 315 (三Q0116 - 021). Later on, Véron-Cetty et al. (1988) redetermined the redshift of UM 324 as being $z=0.355$, a value identical to the one we derived.

A second Parkes radiosource is present in our field (Ekers 1969): PKS $0122-00(3) \equiv 4 \mathrm{C}-00.10 \equiv \mathrm{UM} 321$ which is a quasar with a redshift $z=1.070$ (Lynds 1967). We did not detect it, as is also the case for a second quasar from the UM survey: UM 320 at $z=2.28$ (McAlpine \& Feldman 1982). Finally we did not detect UM 312 which is a possible QSO which seemingly never underwent a spectroscopic identification. 
Table 11. Surface density of ultraviolet-excess quasars per square degree and per 0.5 magnitude bin

\begin{tabular}{lcccccc}
\hline $\begin{array}{c}\text { Magnitude } \\
\text { range }(B)\end{array}$ & $\begin{array}{c}\text { Braccesi et al. } \\
(1980)\end{array}$ & $\begin{array}{c}\text { Boyle et al. } \\
(1987)^{(1)}\end{array}$ & $\begin{array}{c}\text { Boyle et al. } \\
(1990)\end{array}$ & $\begin{array}{c}\text { Cristiani et al. } \\
(1991)^{(1)}\end{array}$ & This work \\
\hline $17.0-17.5$ & 0.12 & - & - & - & 0.15 & \pm 0.08 \\
$17.5-18.0$ & 0.33 & 0.22 & 0.28 & - & 0.24 & \pm 0.11 \\
$18.0-18.5$ & 0.89 & 0.70 & 0.70 & 0.54 & 0.68 & \pm 0.18 \\
$18.5-19.0$ & 2.40 & 2.23 & 2.56 & 1.45 & 0.58 & \pm 0.17 \\
$19.0-19.5$ & 6.49 & 5.58 & 5.29 & 3.59 & 0.53 & \pm 0.16 \\
$19.5-20.0$ & 17.54 & 8.07 & 6.88 & - & $0.73^{(2)}$ & \pm 0.19 \\
\hline \hline
\end{tabular}

1 Interpolated counts.

${ }^{2}$ For $B>19.5$.

At the end of 1987, a preliminary version of the data published here was made available via EG's thesis (Gosset 1987a) and also via the fourth version of the Véron-Cetty \& Véron catalogue (1989).

Part of the NGC 450 field has been surveyed in the framework of the LBQS survey (Chaffee et al. 1991). They found, within the common region, 11 strictly LBQS QSOs. Among these 11 QSOs, 9 were primary candidates in our survey, one was a tertiary candidate $(U-B=-0.20)$ and we did not detect the last one. Chaffee et al. (1991) redetermined the redshifts of our 9 primary candidates; brute comparative results are given in Table 12. One sees that the agreement is rather good except perhaps for the redshift of Q0109-014. The discrepancy is most probably due to the effect of the absorption component on the C IV line (see Table 8). It is surprising to notice that Chaffee et al. (1991) did not detect Q0107-025B (\#209), the twin of Q0107 - 025A (\#210) (see Surdej et al. 1983; Swings et al. 1985; Surdej et al. 1986) whereas they detected the latter one (see Table 12). Chaffee et al. (1991) also reported the existence of two faint non-LBQS QSOs $\mathrm{Q} 0107-0031(z=1.753)$ and Q0108 $+0030(z=0.428)$ that we did not detect.

After inspection of different catalogues and databases, we noticed that two more quasars present in the NGC 450 field remained undetected; these are MS 0114.3-0123 ( $V=19.44, z=0.559)$ discovered as an Einstein Medium Sensitivity Survey (EMSS) X-ray source (Stocke et al. 1991) and Q0112-014 ( $B \simeq 20.3, z=2.20)$ discovered through a grism or grens survey in EMSS fields (Anderson \& Margon 1987). The latter is too faint for the present survey; therefore, we derive a figure of 6 for the number of known quasars missed by our survey (UM $321 \equiv$ PKS $0122-00(3)$, UM 320, MS 0114.3-0123, Q0110 - 0009, Q0107 - 0031 and Q0108 + 0030). If their redshifts are potentially accessible for $U / B$ detection techniques, their exact $U-B$ colours remain however unknown.
Table 12. Cross-identification and comparison between the LBQS and the present survey. Odd rows correspond to LBQS data whereas even ones correspond to our data

\begin{tabular}{|c|c|c|}
\hline Object name & Redshift & Magnitudes $\left(\begin{array}{c}B_{\mathrm{J}} \\
B\end{array}\right)$ \\
\hline Q0107 + 0022 & 1.968 & 18.5 \\
\hline $\mathrm{Q} 0107+003(\# 27)$ & 1.968 & 18.30 \\
\hline Q0107 - 0128 & 1.455 & 18.4 \\
\hline \#151 (tertiary) & - & 18.00 \\
\hline Q0107 - 0235 & 0.958 & 18.1 \\
\hline Q0107 - 025A (\#210) & 0.960 & 17.80 \\
\hline Q0107 - 0232 & 0.728 & 18.4 \\
\hline Q0107 - 025D (\#208) & 0.726 & 18.85 \\
\hline $\mathrm{Q} 0108+0028$ & 2.005 & 18.3 \\
\hline Q0108 + $004(\# 28)$ & 2.009 & 18.25 \\
\hline Q0109 - 0128 & 1.758 & 18.3 \\
\hline Q0109 - 014 (\#153) & 1.746 & 18.05 \\
\hline Q0110 - 0107 & 1.889 & 18.6 \\
\hline Q0110 - $011(\# 126)$ & 1.887 & 18.80 \\
\hline Q0110 - 0157 & 1.102 & 17.2 \\
\hline Q0110 - 019 (\#175) & 1.099 & 17.30 \\
\hline Q0110 - 0015 & 0.976 & 18.4 \\
\hline Q0110 - 002B (\#82) & 0.968 & 18.55 \\
\hline Q0110 - 0009 & 1.686 & 17.8 \\
\hline- & - & - \\
\hline Q0110 - 0047 & 0.412 & 18.7 \\
\hline Q0110 - 007 (\#104) & 0.411 & 18.90 \\
\hline
\end{tabular}




\section{Spatial distribution of the quasars}

Although our set of discovered quasars is relatively small and, thus, cannot by itself provide a definitive answer to the general problem of the spatial distribution of quasars, we consider it useful to independently analyse the present isolated dataset in order to reach a level of knowledge as high as possible on our sample. For the sake of completeness of our subsequent analysis, we define three subsamples that we consider separately: the first one, labelled A1, contains all the 140 primary and secondary candidates; the second one, A2, is related to the 95 primary candidates alone whereas the one labelled A3 deals with the 62 extragalactic objects identified (59 quasars and 3 possible Seyfert 2 galaxies or assimilated). The full analysis is detailed in Gosset (1987a); a complete summary is given here. A first look will be devoted to the 2-D spatial distribution of the objects on the celestial sphere.

\subsection{The 2-D distribution of the quasars in $\alpha, \delta$}

The objects in our samples have been projected on a plane using the equal area projection introduced by Webster (1976b). Figure 6 presents the projected field. The human eye immediately notices clusters and voids. We will particularly retain the strong association (5 quasars) at the edge of the field in the WSW direction as well as the void in the SSW direction. Whereas a visual inspection may be very powerful in detecting anomalies, only a quantitative approach will help to know whether the observed effects are attributable to the underlying process or are simply due to statistical fluctuations of the sampling. In order to do this, one should make use of a wide variety of statistical methods. Indeed, each method has its own advantages, sensitivity, properties but also has its defects, weaknesses and biases. In addition, some interpretations are quite intricate (see also Gosset et al. 1988). Therefore, an exhaustive knowledge or understanding of the data is not accessible when one uses only one method. The combined results of several independent methods are, without any doubt, essential to reach a high degree of confidence in the conclusions of the analysis. Usually, the nullhypothesis which is tested corresponds to a very simple concept. On the opposite, the complementary to the nullhypothesis, the global alternative hypothesis, is complex, composite. In the case of a rejection of the null-hypothesis, each method will point towards a particular subset of the global alternative hypothesis; the use of several methods and, subsequently, the critical combination of the different subsets will allow a better determination of the true cause of the rejection.

The application of the Extended Kolmogorov-Smirnov (EKS) test introduced in its 2-D version by Peacock (1983; see also Gosset 1987b) teaches us that subsamples A2 and A3 are in perfect agreement with the null-hypothesis of uniform randomness. For its part, subsample A1 is tied

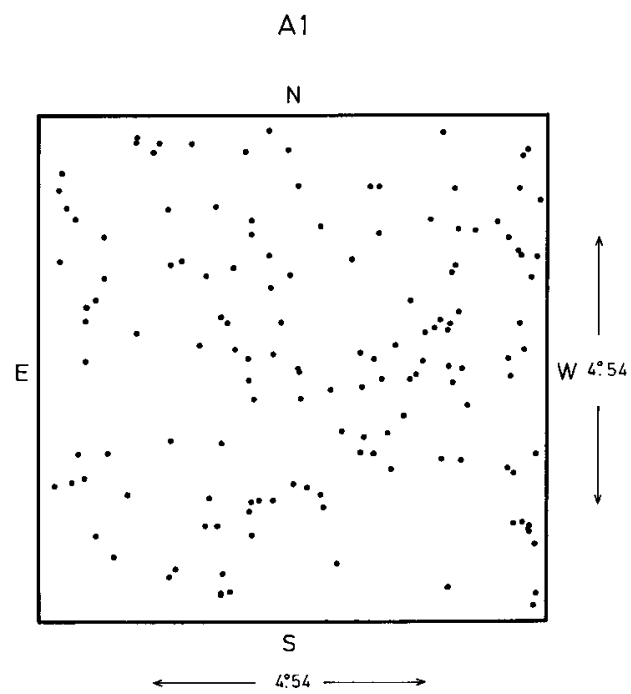

A 2

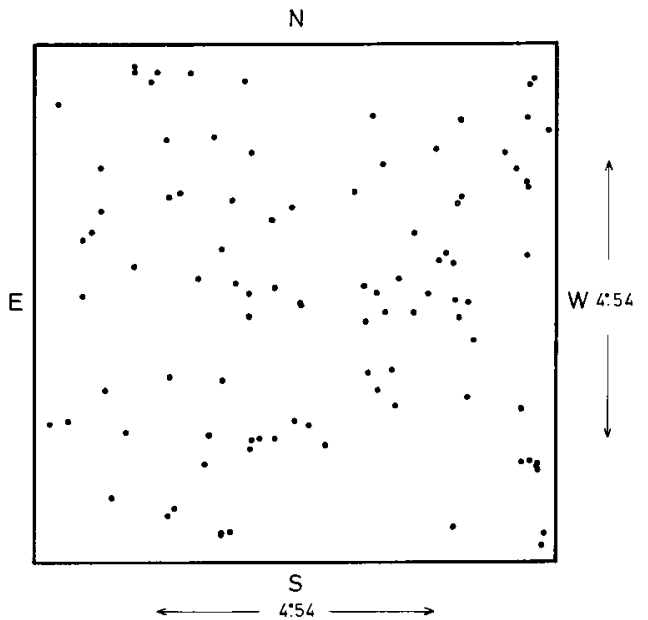

$A 3$

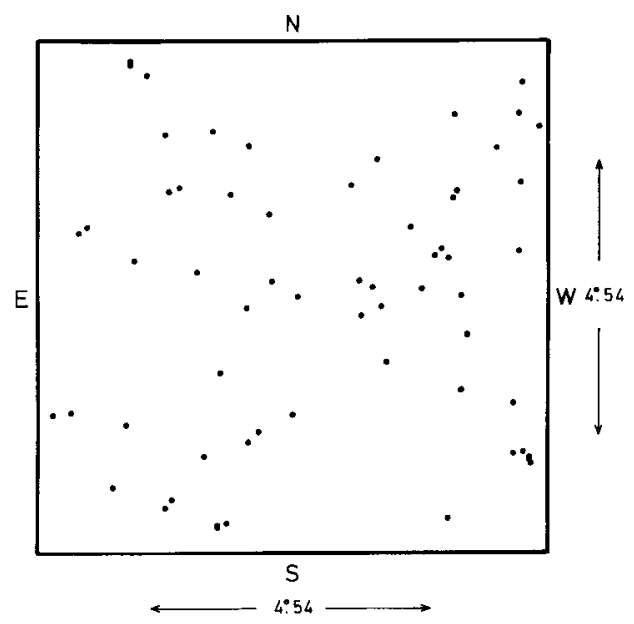

Fig. 6. Distribution of the quasar candidates of the different samples in the projected area of the field (A1: 140 primary + secondary candidates, A2: 95 primary candidates, A3: 62 quasars and related AGN) 
in with a significance level ( $S L$ : probability to observe such a deviation under the adopted null-hypothesis) of 0.072 which suggests a slight anomaly. We tried to modify the null-hypothesis by considering non-constant probability density functions (pdf) symmetrical with respect to the centre of the field (normal distribution; $\mathrm{e}^{-\alpha R}$ law following Peacock 1983, where $R$ is the distance from the plate centre) in order to search for a better fit while putting in evidence a possible centre-to-edge effect. None of our trials turned out to be successful, suggesting that one has to deal with a non-localized effect. The position of the largest deviation between the cumulative distribution function (cdf) and the sample distribution function (sdf) seems to indicate that the anomaly is associated with the above-mentioned strong association and void.

\subsubsection{The Multiple Binning Analysis (MBA)}

The MBA is one of the most ancient methods and a review of the different associated tests of significance is given in Gosset \& Louis (1986). The latter also introduced a powerful 2-D test called the 4 within 16 randomization test which analyses the degree of correlation between the counts in adjacent bins. The corresponding normal $Z$ statistic (positive in case of deviation towards clustering, negative in case of regularity) is given in Fig. 7 as a function of the number of pairs of basic bins. For subsample A1, we note three deviations:

$-1 / 128$ of the field side size, i.e. about 2 arcmin $(S L=0.067)$;

$-1 / 32$ and $1 / 16$ of the field side size, i.e. about 8.5 and $17 \operatorname{arcmin}(S L$ of 0.122 and 0.054 , respectively);

$-1 / 4$ of the field side size, i.e. a little more than one degree $(S L=0.0007)$.

This last point is in good agreement with the results from the EKS test. For subsample A2, we retain 1/32 (8.5 arcmin, $S L=0.073)$ and $1 / 4\left(\simeq 1^{\circ}, S L=0.022\right)$ and for subsample A3, only $1 / 32(8.5 \operatorname{arcmin}, S L=0.046)$. All the deviations are towards positive $Z$ values indicating a deviation from randomness towards clustering.

\subsubsection{The Correlation Function Analysis (CFA)}

The CFA consists in estimating the underlying pair autocorrelation function versus the angular separation $\theta$, by making counts of objects in concentric rings sequentially built-up around each object of the sample. Such a method is extremely sensitive to edge effects and a correction has to be applied. The only way to properly perform this correction is to calculate the exact measure of the domain actually explored when making counts (i.e. to reject the area of the ring situated out of the investigated field). This approach can however induce systematic effects and the best way to estimate them consists in computing the mean cross-correlation function between simulated populations of uniformly distributed individuals (representing
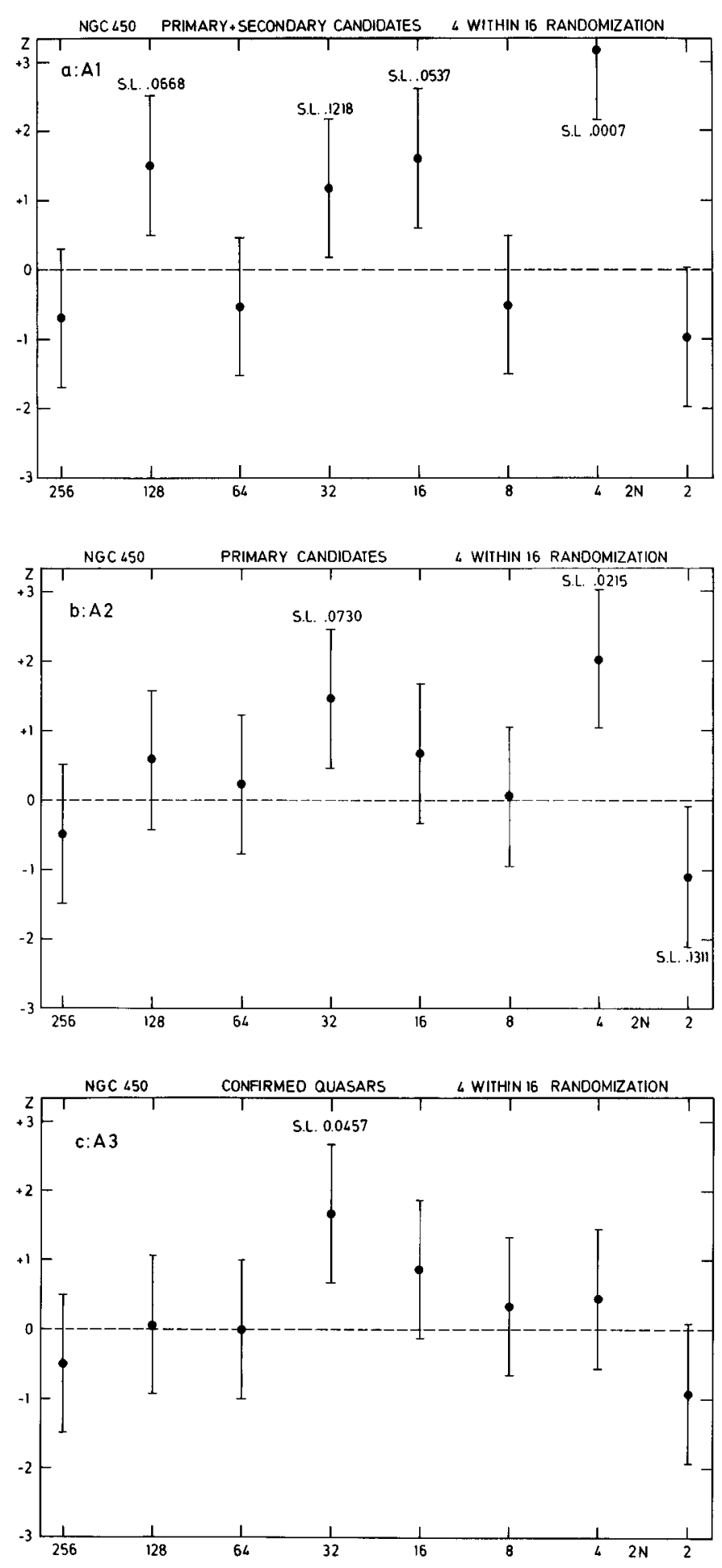

Fig. 7. Runs of the $Z$ statistic of the MBA (4 within 16 randomization test) as a function of half the number of basic bins on one side $(1 / 2 \times 4 N=2 N)$. The explored characteristic scale is given by $4.54 / 2 N$. The three panels correspond to the three samples A1, A2 and A3. The $S L$ associated with some deviations of interest is also given 
the null-hypothesis) and the data (as suggested by Sharp 1979). One of the estimators, the one corresponding to the true objects taken as centres and the counts performed on the simulated individuals (labelled $w_{\text {data/random }}$ ), is unbiased and its dispersion can be used to estimate the error on the correlation function. The other estimator (labelled $\left.w_{\text {random/data }}\right)$, corresponding to the reverse operation, contains the artefacts of the edge corrections. It should directly be compared with the autocorrelation estimator of the data $w_{\text {data/data }}$.

The results of the CFA are given in Fig. 8. One clearly sees that the artefacts of the edge corrections are rather low. There is an overdensity of objects at small $\theta(<0.2)$ in all the three sets; this indicates a clustering at a scale of the order of 12 arcmin. The deviations are approximately $8 \sigma$ (A1), $2.9 \sigma$ (A2) and $5 \sigma$ (A3): this clustering is significant, a result in good agreement with the conclusions of the MBA. We have also estimated the deviations relatively to the standard deviations $\sigma^{\prime}$ computed on the basis of the amount of independent pairs. In this context, we obtain deviations of $2.1 \sigma^{\prime}, 2.5 \sigma^{\prime}$ and $3.7 \sigma^{\prime}$, respectively. We know that, in this case, the dispersion is usually overestimated. The clustering is undoubtedly present in the A3 subsample. No significant deviation exists at other scales although a slight overdensity is suggested for separations $\theta \in[0.4,0$. 6$]$.

\subsubsection{The Nearest Neighbours Analysis (NNA)}

The NNA is based on the analysis of the mean distances to $n^{\text {th }}$ nearest neighbours (see Clark \& Evans 1954; Gosset 1987a; Rose 1977). It is sensitive to the edge effects but no rigorous CFA-type correction can be easily applied. This is partly due to the fact that the NNA statistics also depend on higher order terms of the correlation function. An approximation can be obtained by using simulated populations mimicking the data, provided that the simulations do reproduce correctly some of the characteristics of the analysed population. For want of something better, we compared the mean distances to neighbours within the subsamples with those within simulated uniform populations. This type of correction could be slightly inappropriate, at least for the A1 subsample, since the genuine population could be non-uniform at large scales. For all the three subsamples, the first nearest neighbours (up to the fourth one) are too close comparatively to the nullhypothesis values. The corresponding $S L$ is never lower than 0.05 , so that no firm conclusion can be drawn from the NNA alone. However, the NNA points out to a deviation towards clustering in good agreement with the previous analyses. Indeed, the mean distance to the first nearest neighbour is of the order of 10 to 20 arcmin depending on the sample considered. This suggests that the abovementioned clustering at $\simeq 10$ arcmin is mainly due to pairs of individuals.
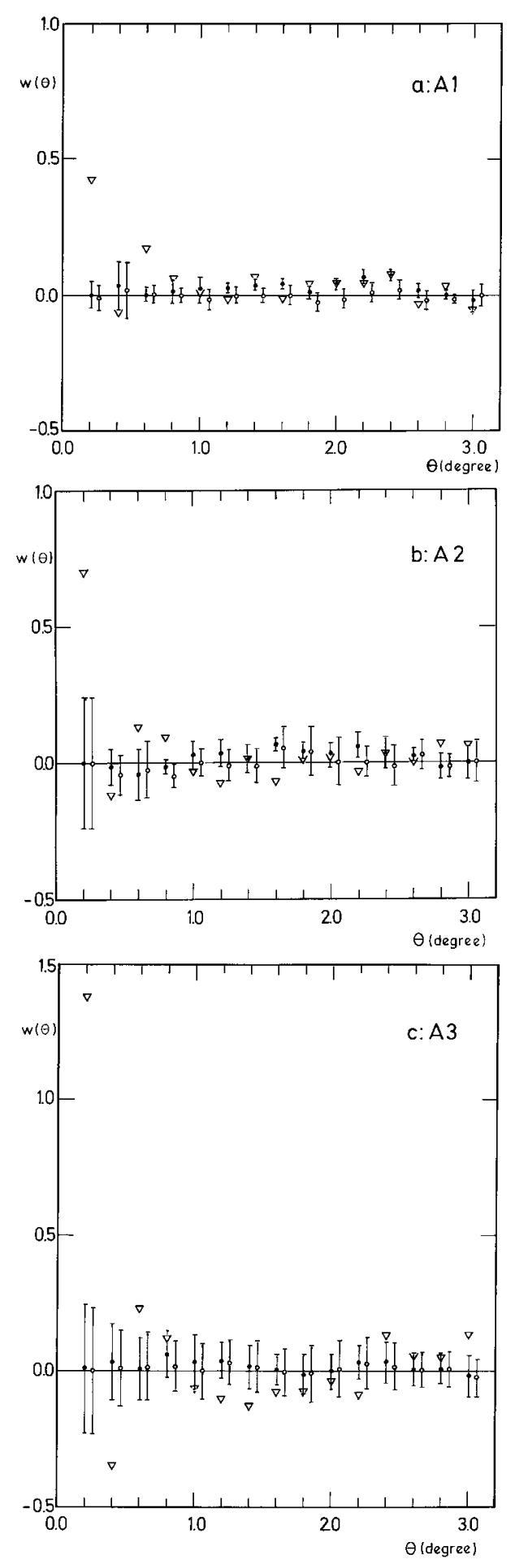

Fig. 8. Results of the bi-dimensional CFA. Estimated autocorrelation function as a function of $\theta$, the characteristic angular separation expressed in degrees. The triangles represent the function $w_{\text {data/data }}$; the filled circles represent the function $w_{\text {random/data }}$ and the open circles the function $w_{\text {data/random }}$. The latter is displaced along the abscissa for the sake of clarity. The error bars give \pm 1 standard deviation of the dispersion over the simulations 


\subsubsection{The Power Spectrum Analysis (PSA)}

The PSA (Webster 1976a) has a good reputation of flexibility and of great sensitivity, although Gosset et al. (1988) illustrate a problem of intricate interpretation. The PSA is based on Fourier transformation of point processes. In the case of the present data, the $Q^{\prime}$ statistic (Webster 1976a) deviates from randomness only for spatial frequencies smaller than $1 / \lambda \simeq 5$ degree $^{-1}$, but for these frequencies strictly all the $Q^{\prime}$ statistics deviate towards larger values. This result is valid for all the subsamples. Again, this indicates deviations towards clustering with a typical scale around 15 arcmin. The global $Q$ statistics we derived are

$$
\begin{array}{ll}
\text { A1: } & \lambda \simeq 0.3^{\circ}(18 \text { arcmin }) \\
& Q=1.18,2 U=716, S L=0.0006, \\
\text { A2: } & \lambda \simeq 0.23^{\circ}(13 \text { arcmin }) \\
& Q=1.16,2 U=1272, S L=0.0001, \\
\text { A3: } & \lambda \simeq 0.23^{\circ}(13 \text { arcmin }) \\
& Q=1.25,2 U=1272, S L \ll 0.0001,
\end{array}
$$

where $2 U$ is the number of degrees of freedom (Webster 1976a).

The $Q$ statistics and the $S L$ reported above refer to all the scales equal to or larger than the one given. Therefore, they are not univocally linked to the $10-15$ arcmin clustering but are influenced by the situation at larger scales. This could explain why the $S L$ are so small. However, the MBA detects no other deviation for subsample A3: the clustering at $10-15$ arcmin is thus probably the main cause in this case. We can estimate a reasonable upper limit on the $S L$ linked to this clustering by adopting for all the necessary scales a typical $Q^{\prime}$ value of 1.15 as derived from the deviating $Q^{\prime}$ value at the largest spatial frequencies. For a value $2 U=716$, these $Q^{\prime}$ values lead to an $S L$ of 0.003 , much more secure and realistic as an upper limit. The above conclusions are independent of the inclusion or exclusion of the 1-D terms in the computation of the 2-D PSA statistic.

Concerning the 2-D clustering analysis, we may conclude that a clustering of quasars is clearly present at a scale of about 10 arcmin. It is possible to detect a few groups that participate to this clustering. Let us mention

- Q0107 - 025A, Q0107 - 025B and Q0107-025D; we can also add Q0107 - 025C and Q0108-025. We will particularly notice the pair of quasars Q0107 - 025A and Q0107 - 025B, separated by 77 arcsec, and with a very small difference in redshift $(\Delta z=0.004)$. A detailed study of this pair has been published by Surdej et al. (1986).

- Q0118 - 031B and Q0118 - 031C with quite different redshifts. One could also add Q0118 - 031A situated some 4.8 arcmin farther. The triplet has been studied by Robertson et al. (1986).

- Q0121+009 and Q0121+008, separated by 2.8 arcmin.
- Q0110 - 002A and Q0110 - 002B, separated by 3.7 arcmin.

- Q0110 - 006 and Q0111 - 007, separated by 5.2 arcmin and with a redshift difference of $\Delta z=0.06$. These two quasars have a redshift of $\simeq 0.9-1.0$, just like Q0107$025 \mathrm{~A}$ and $\mathrm{Q} 0107-025 \mathrm{~B}$.

- Q0120 - 029A and Q0120 - 029B, separated by 5.3 arcmin.

- Q0123 - 005A and Q0123 - 005B, separated by 5.9 arcmin.

Although the objects of this list do participate in the detected clustering, none of the associations can be labelled as being significantly abnormal on its own.

\subsection{The 1-D distribution of the redshifts}

We will study here the distribution of the redshifts of the objects of subsample A3. The related histogram is given in Fig. 9. The main striking feature is the presence of an outstanding excess of objects with a redshift between 0.9 and 1.0; this has first been reported by Swings et al. (1983). Other regions with high density are situated around $z=2.0, z=1.3$ and $z=0.4$. These inhomogeneities are most likely due to a selection effect, although an alternative explanation can be found in Arp et al. (1990). Indeed, the emission lines are determining on the colours of quasars. The quasar will look bluer, and will be easier to detect in our survey if a strong line is present in the $U$ band. Good examples are the Ly $\alpha$ line (for $z \simeq 1.9$ ), the $\mathrm{C}$ IV resonance doublet $(z \simeq 1.3)$, the $\mathrm{Mg}$ II analog $(z \simeq 0.3)$ and possibly the $\mathrm{C}$ III] intercombination line $(z \simeq 0.9)$. The opposite effect exists when such a line enters the $B$ band. All this should determine the general appearance of the histogram except for the peak at $z \simeq 0.9-1.0$ which is most probably too narrow and too well-marked to be fully explained by a similar effect.

If we adopt, as a null-hypothesis, the uniformity of the distribution of objects with $z<2.3$, a KolmogorovSmirnov test associates an $S L$ of 0.05 to our sample. In order to model the above-mentioned observational biases, we tried to estimate the $U-B$ colours of quasars as a function of redshift. Then we arbitrarily fixed the probability to detect the quasar as being proportional to the $U-B$ excess. Another approach is to look at the distribution of quasars selected by the same method in current catalogues. Qualitatively, both ways lead to similar results. We thus derived a new pdf taking into account these observational biases. This new adopted pdf clearly exhibits three maxima at $z=0.3,1.3$ and 1.9 , but lacks for evidence of strong effects attributable to the C III] line. A Kolmogorov-Smirnov test with this new null-hypothesis leads to its acceptance, which is no clue of the prime cause of the effect.

Application of the PSA to this 1-D sample reveals a deviation towards regularity with a typical scale of 0.4 


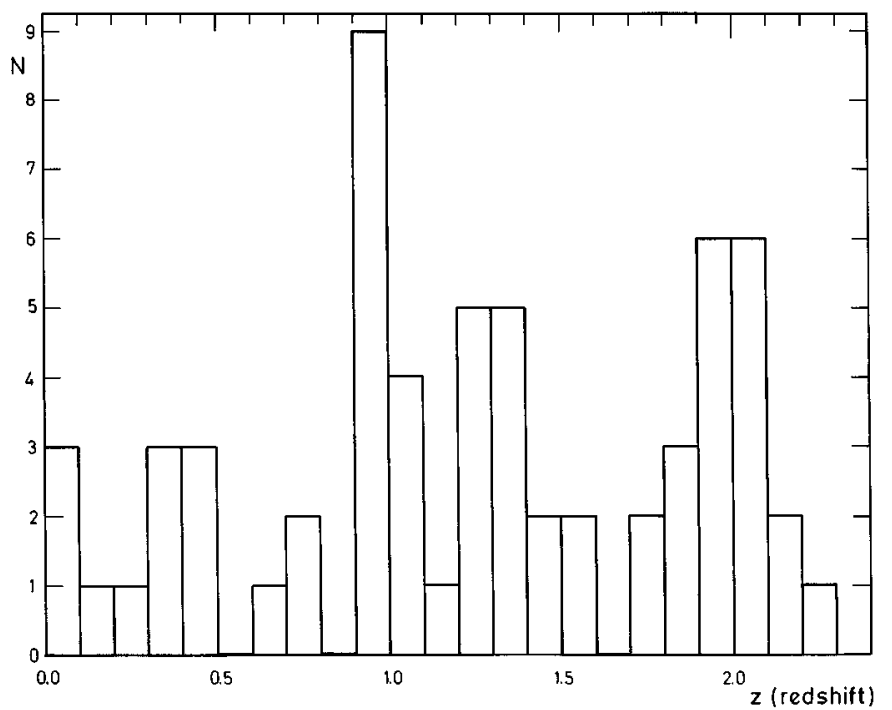

Fig. 9. Histogram of the distribution of redshifts of the quasars and assimilated objects; note the surprising excess in the bin $z \in[0.9,1.0]$

to 0.5 in redshift. This again corresponds to the abovementioned observed effect. If we now adopt the new nonconstant pdf as the null-hypothesis, we have to make use of the GPSA (Generalized PSA; see Peacock 1983). The run of the GPSA $Q^{\prime}$ statistic as a function of the spatial frequency $1 / \lambda$ (in the redshift domain) is given in Fig. 10. Only the first point does exhibit a marked deviation $(S L=0.015)$. This suggests that the pdf is not fully adapted, which is no surprise owing to the arbitrariness of its definition; the situation is however improved compared to the PSA case. The second and the third points do not significantly deviate when taken individually. But they could indicate a clustering with a typical scale $\lambda \simeq 0.11$, an effect to be related to the peak at $z \in[0.9,1.0]$. Nevertheless, the deviation is not significant, corresponding to a global $Q=1.18$ with 42 degrees of freedom. Contrary to what was done in Swings et al. (1983), the present test is basically continuous and has been applied to a well-defined sample. Also, we should not forget that one quasar present in the peak has an uncertain redshift and that only three of them have redshifts confirmed by other authors. In any case, this anomaly deserves further consideration. Full completion of our different surveys may bring some clues to the origin of the phenomenon.

\subsection{The 3-D distribution of the quasars}

The three-dimensional - truly spatial - distribution of our quasars can also be investigated. We need for that an interpretation for the redshifts that could relate them to distances, through a cosmological model. The lack of observational evidence towards any of the available models, suggests to use the simplest one. We therefore adopted a

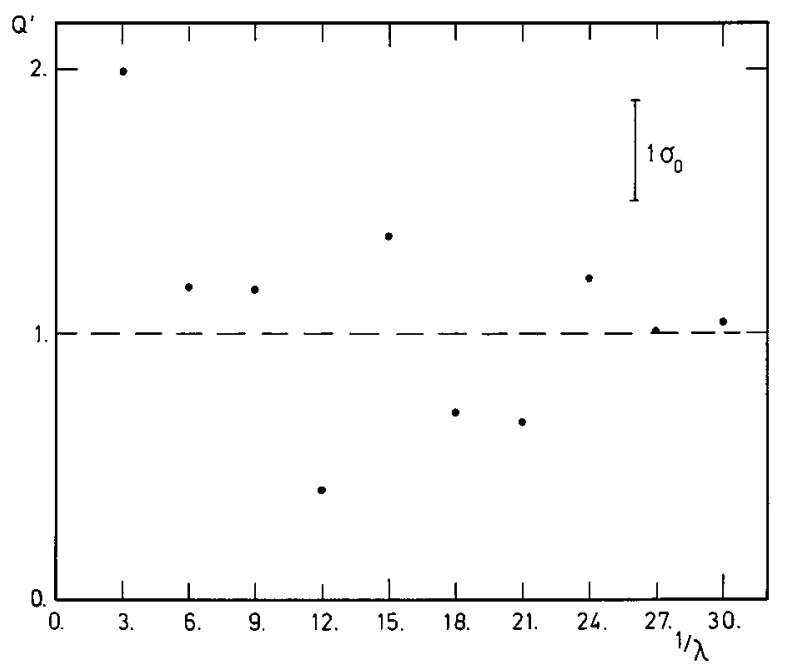

Fig. 10. Run of the $Q^{\prime}$ statistic of the 1-D GPSA as a function of the spatial frequency (in the redshift domain); the adopted pdf is discussed in Sect. 6.2 and the analysed sample is A3

Friedmann-Robertson-Walker (FRW) model without cosmological constant $(\Lambda=0)$ and with a deceleration parameter $q_{0}=1 / 2$ corresponding to a euclidian geometry (Einstein-de Sitter Universe). The Hubble constant is taken to be $H_{0}=100 \mathrm{~h} \mathrm{~km} \mathrm{~s}^{-1} \mathrm{Mpc}^{-1}$. The choice of the model and of the constants is arbitrary; the results of the clustering analysis are not completely independent of this choice but they are not too sensitive either. In the parametric neighbourhood of the chosen FRW model, the impact of the choice of $q_{0}$ becomes just a matter of a scale factor, as is always the case for $H_{0}$. The objects have been positioned in such a comoving space; they are all contained in a $0.2 \times 0.2 \times 2.5 \mathrm{~h}^{-3} \mathrm{Gpc}^{3}$ parallelepipedic volume. But the one actually explored is more reminiscent of a truncated pyramid. Practically, the volume is very elongated and the low 3-D density of objects does not authorize a fine analysis.

We adopted a pdf keeping a constant value within the truncated pyramid and zero outside. Application of the 3-D EKS test (see Gosset 1987b) leads to the acceptance of the null-hypothesis of uniformity: the non-uniformities in redshift seem to be diluted here. This is no surprise since the 1-D EKS test (see Sect. 6.2) was already reporting no strong anomaly; the addition of two spatial dimensions is expected to wash out the effect. Application of the GPSA with the same pdf detects no anomaly either, but the analysis is safely restricted to scales of 65 to $200 \mathrm{~h}^{-1} \mathrm{Mpc}$.

For its part, the application of the MBA (in its version of the 8 within 64 randomization test; see Gosset \& Louis 1986) is delicate for two related reasons. First, the expectation of the counts in each cell is very low. Second, the basic bin has, like the full domain, one side 12.5 times greater than the two other ones, implying a mixing of the scales. A brute application of the test suggests two 


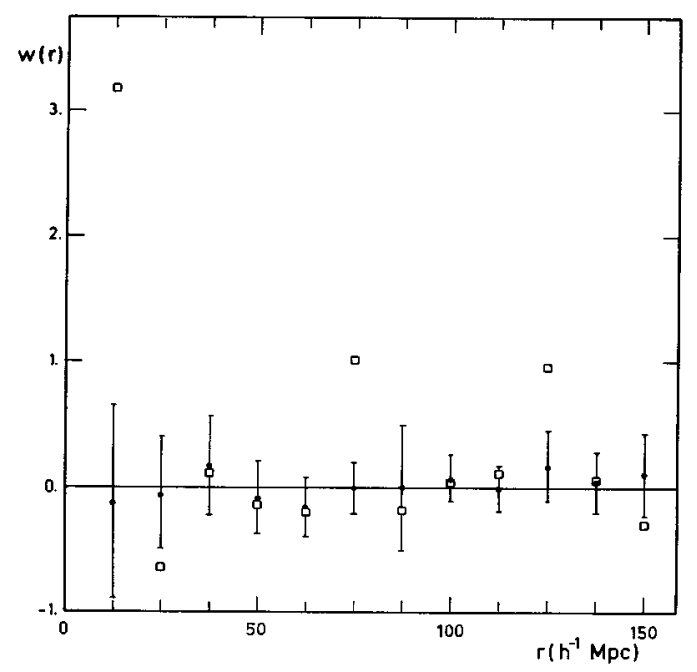

Fig. 11. Result of the three-dimensional CFA for the A4 dataset. Estimated autocorrelation function as a function of $r$, the characteristic separation expressed in $\mathrm{h}^{-1} \mathrm{Mpc}$. The squares represent the function $w_{\text {data/data }}$ and the filled circles the function $w_{\text {random/data }}$. The error bars are those of the function $w_{\text {data/random }}$ and represent \pm 1 standard deviation of the dispersion over the simulations. By random, we mean populations obtained by shuffling the redshift values among the quasars and assimilated objects

deviations towards clustering: the first one, at large scale ( $1 / 4$ of the side), is marked and due to the fact that only the truncated pyramid is populated, and not the full parallelepiped; the second one corresponds to $1 / 32$ of the side which denotes a mixed scale of $6 \mathrm{~h}^{-1} \mathrm{Mpc}$ to $75 \mathrm{~h}^{-1} \mathrm{Mpc}$ $(S L=0.04)$. Seyfert 2 galaxies contribute to the latter.

Application of the CFA is also difficult; the low density does not allow bins smaller than $12.5 \mathrm{~h}^{-1} \mathrm{Mpc}$. In addition, the elongated shape of the domain renders the volume corrections delicate. In particular, the $\mathrm{CFA}$ is dominated by two objects, NGC 450\#24 and NGC 450\#87 which, due to their low redshifts, are rather close to each other and also close to the small basis of the truncated pyramid. This fact induces large volume corrections and artificially increases the weight of these two objects in the estimation of the correlation function. Their removal should not influence our analysis of the quasar distribution and therefore we defined a fourth subsample (A4) containing 60 objects. The CFA of the A4 dataset leads to the detection of two deviations: the first bin at $12.5 \mathrm{~h}^{-1} \mathrm{Mpc}$ is overpopulated, as is also the one at $75 \mathrm{~h}^{-1} \mathrm{Mpc}$. This analysis could be vitiated by the inhomogeneities in redshift. A way to get rid of this effect is to generate the simulated populations used in the computation of $w_{\text {data/random }}$ and $w_{\text {random/data }}$ by shuffling the redshifts among the objects instead of taking the redshift from a random distribution. Such methods were already used by Osmer (1981) and Anderson et al. (1988). We thus break the relation object-redshift keeping necessarily the same redshift distribution.
The result of this CFA for the A4 subsample is given in Fig. 11. The two deviations at $12.5 \mathrm{~h}^{-1} \mathrm{Mpc}$ and at $75 \mathrm{~h}^{-1} \mathrm{Mpc}$ are significant. Due to the low density of the sample, these deviations can be due to a small number of objects. The first bin deviates because of the presence of the true pair of quasars Q0107 - 025A and Q0107 - 025B (which, in addition, is situated close to one of the field edges). The other deviation could be due to two groups of quasars separated by about this distance of $75 \mathrm{~h}^{-1} \mathrm{Mpc}$ such as the above-mentioned pair and the pair Q0110-006 and Q0111-007. Different estimations of the $S L$ related to the deviation of the first bin lead to an upper limit $S L<0.04$. Our 3-D analysis therefore detects a clustering at an $S L$ of 0.04 and with a typical scale of the order of $10 \mathrm{~h}^{-1} \mathrm{Mpc}$. Although formally correct, these results are to be taken cautiously because the corresponding deviation is essentially due to one pair. This means that the deletion of only one well selected object among the 60 or 62 is sufficient to cancel the clustering. Although this fact is not satisfactory, it is the mere consequence of the small size of the analysed sample. Consequently, neither is the typical scale meaningfully definable, nor are we authorized to assign the 3-D clustering property to the quasar population itself. It is interesting to notice that this $10 \mathrm{~h}^{-1} \mathrm{Mpc}$ scale is within a small factor compatible with the projected scale of 10 arcmin reported in the 2-D analysis. However, the 2 -D clustering is not entirely attributable to Q0107-025A and Q0107 - 025B.

\section{Conclusions}

We presented here the results of a survey for quasars based on the ultraviolet-excess selection technique and performed in a 20.6-square-degree field located in the vicinity of NGC 450. The quasar candidates were selected by visual inspection of a $U / B$ dual-exposure Schmidt plate; the survey was photometrically calibrated a posteriori using the MAMA measuring machine. The derived limiting magnitude is $B=19.6$ and the threshold value in $U-B$ is -0.30 . We presented a list of 140 quasar candidates (95 primary +45 secondary) with accurate positions and $U / B$ magnitudes. The survey technique has been designed to favour the homogeneity of the selection all over the plate, whereas the photometric reduction was necessary to derive the limits of the survey. However, the photometric calibration suffers from several problems such as the fact that the faint end of the photometric sequence is restricted to a small area of the plate. Therefore, we consider that the homogeneous output of the survey is our list of candidates despite the given photometric characteristics of the objects.

The primary candidates have been spectroscopically identified, leading to the detection of 59 quasars (out of which only 6 were previously known). This suggests an efficiency of at least $60 \%$. We did not spectroscopically identify the secondary candidates. Instead, we initiated, 

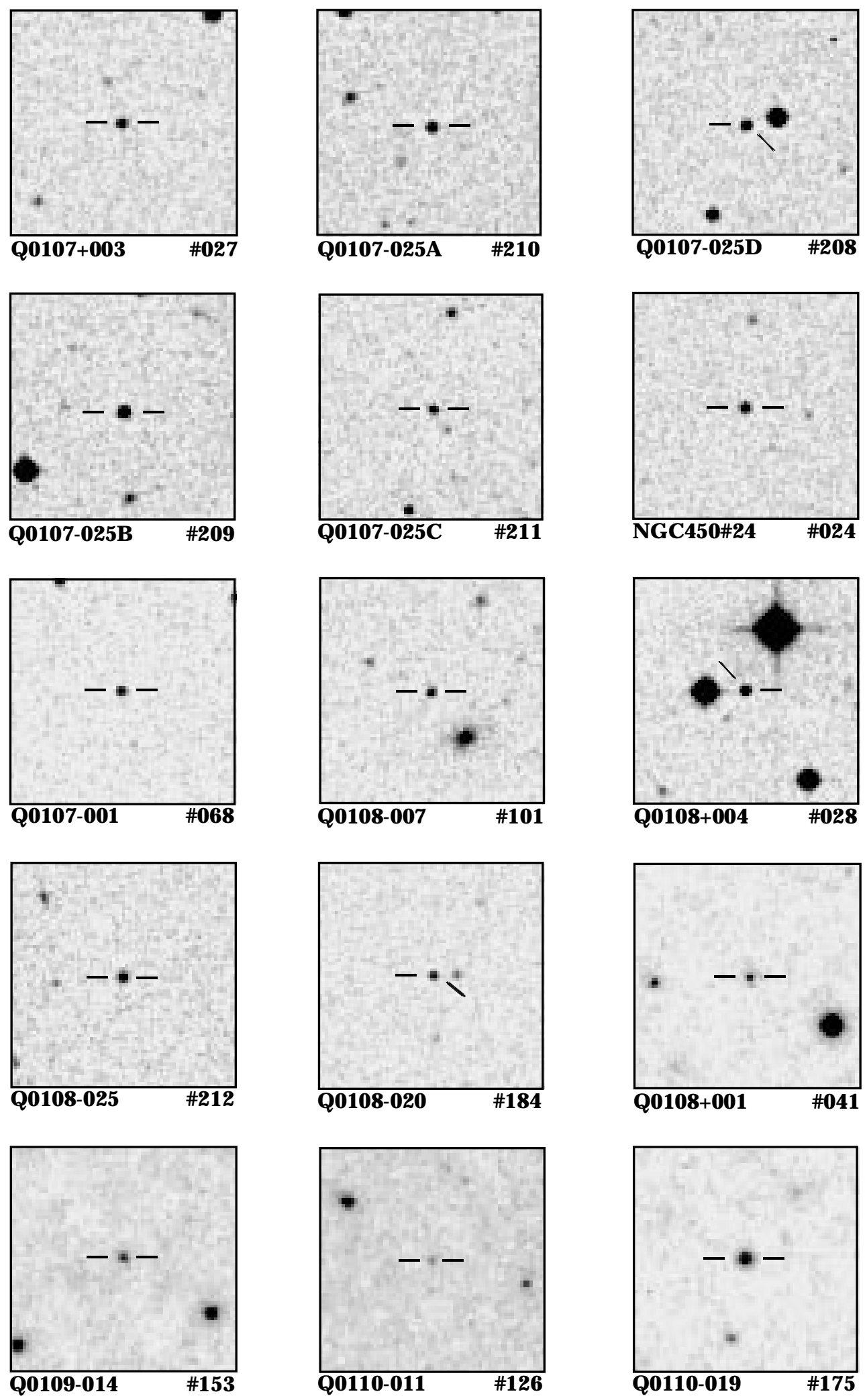

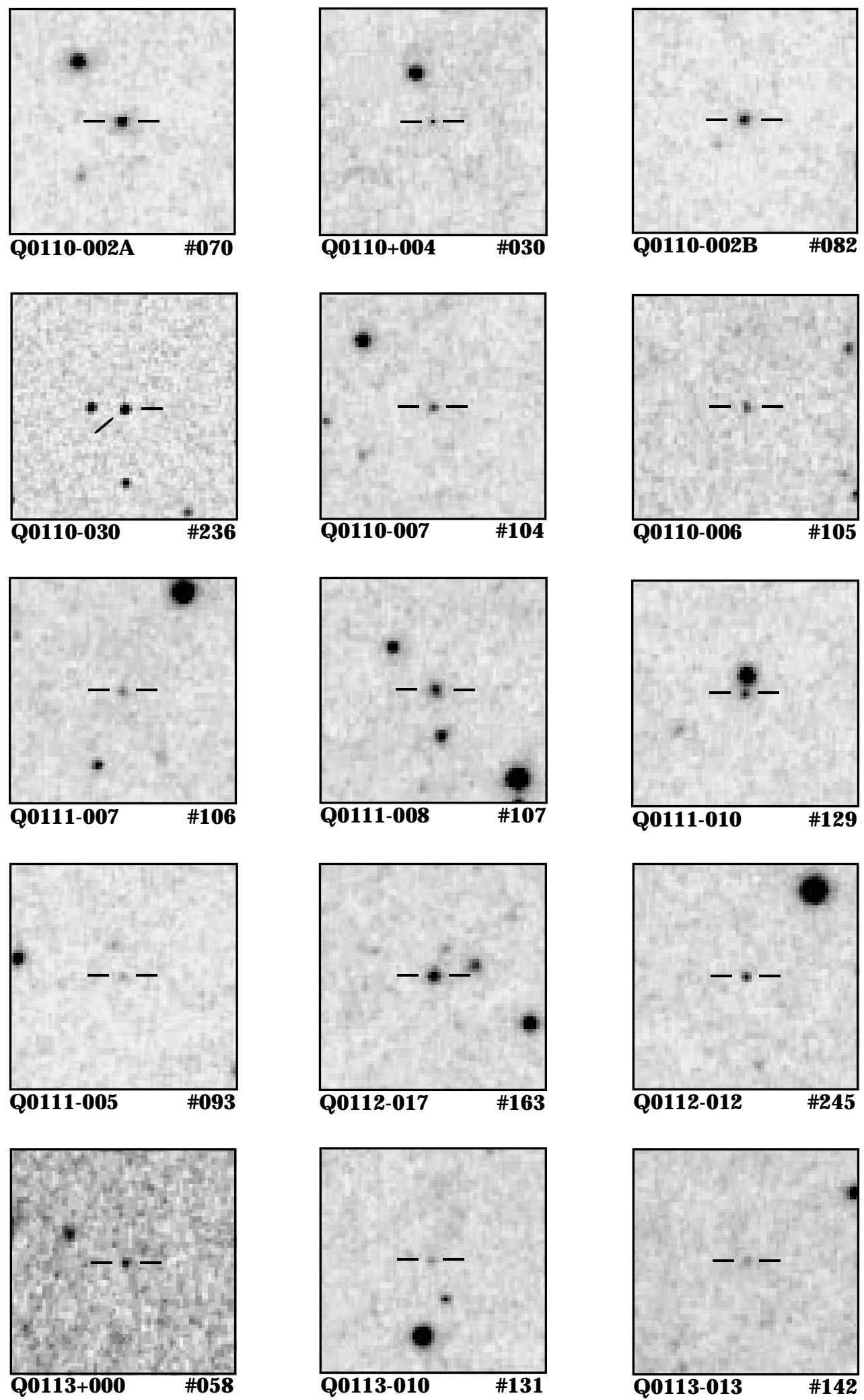

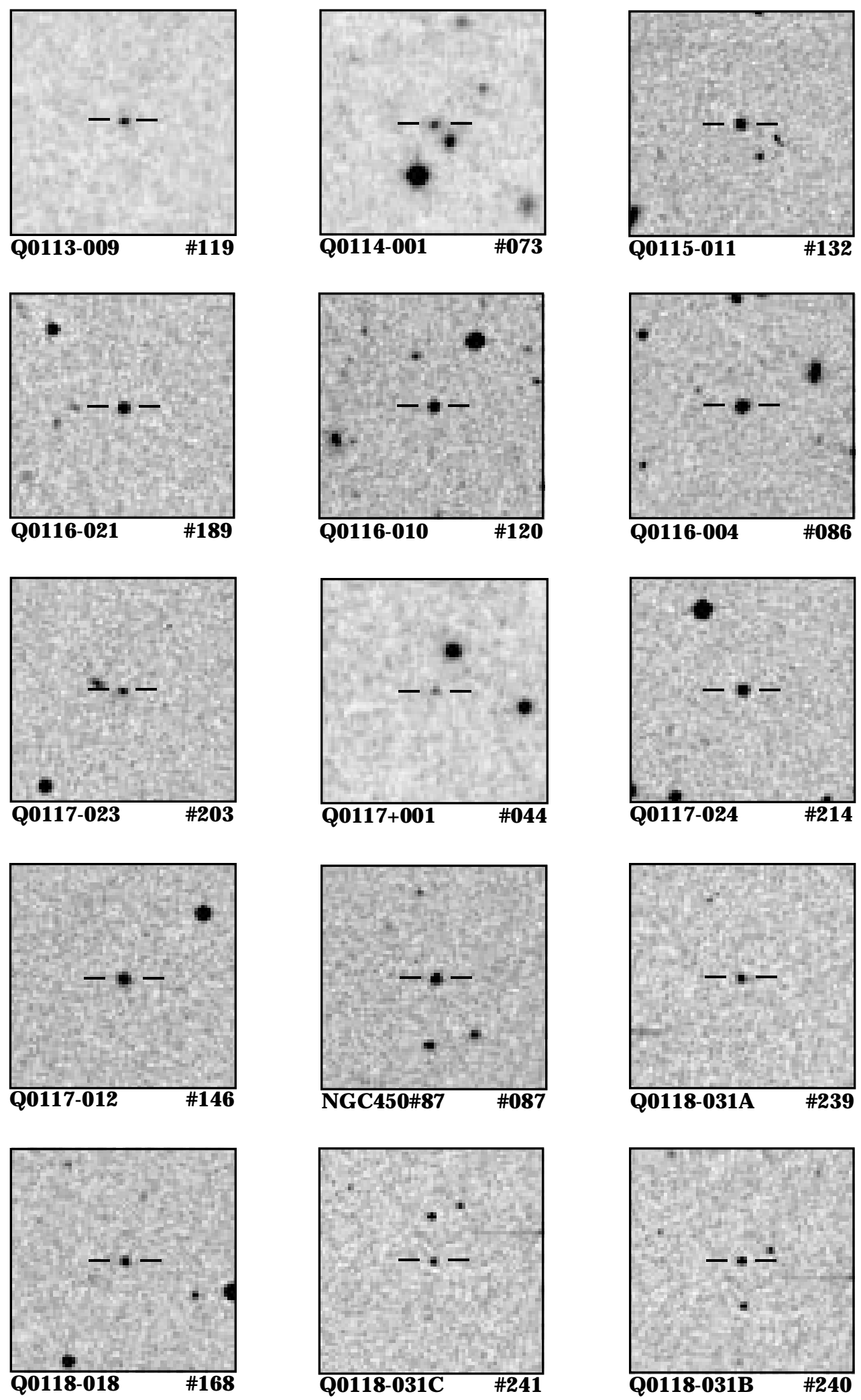

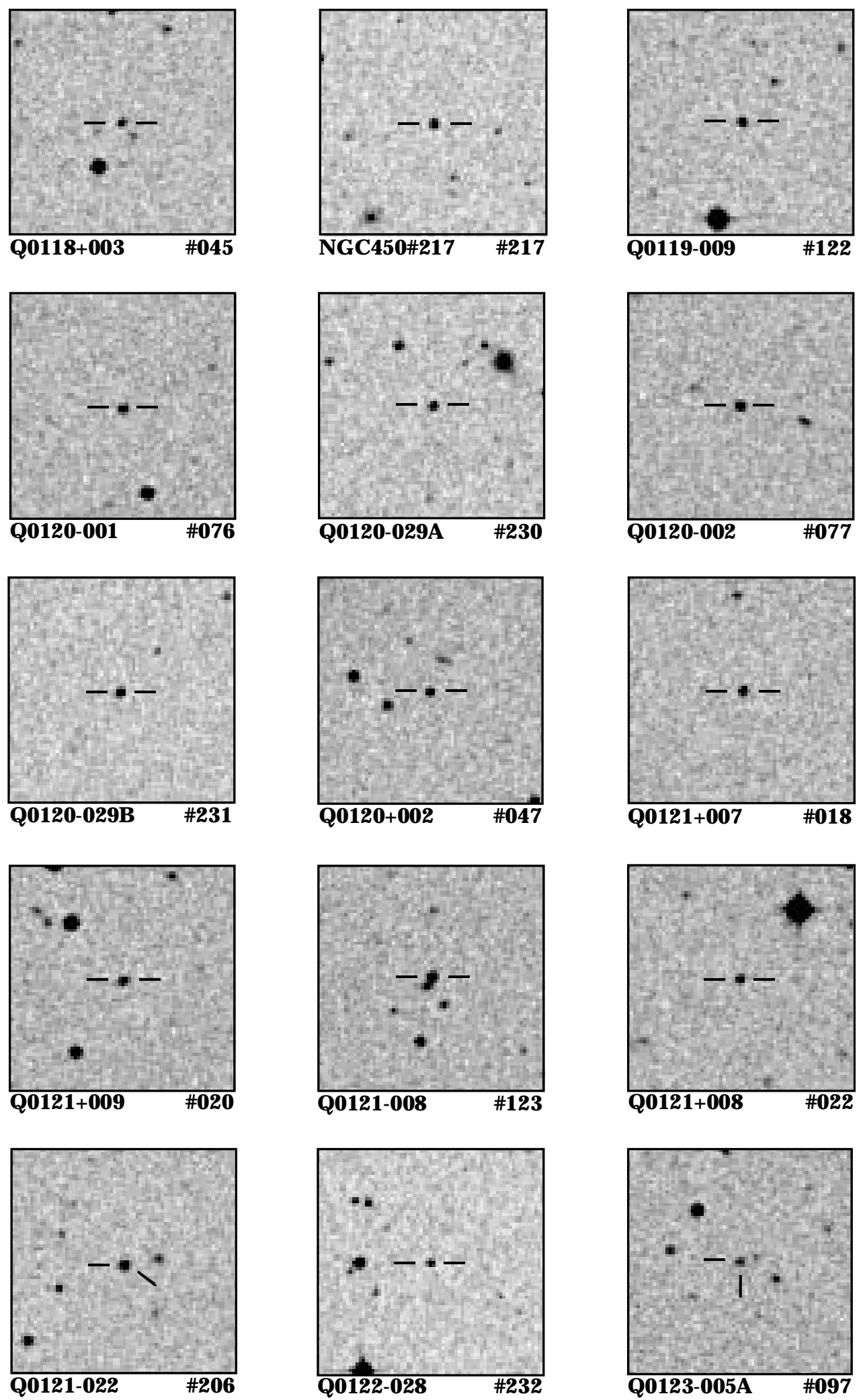

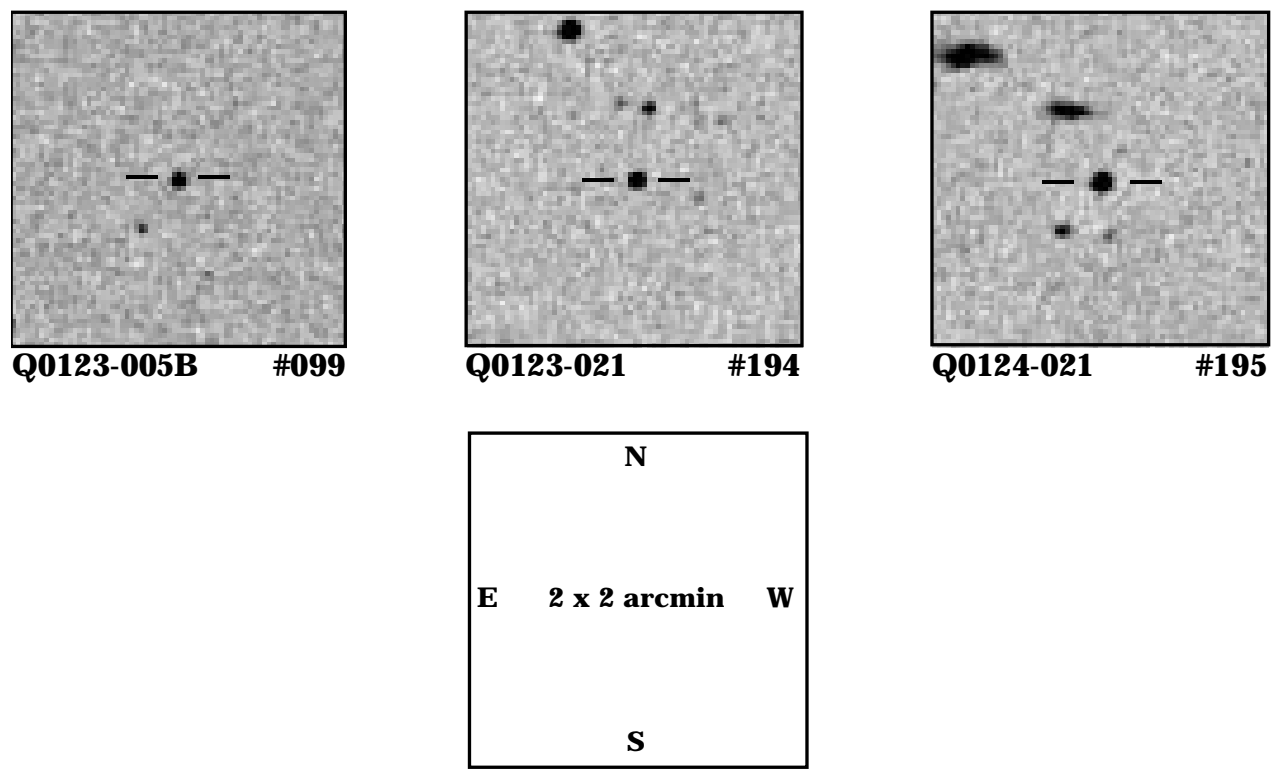

in the same fields (see Sect. 1), quasar surveys based on other selection criteria and we expect that the merging of all the different candidate lists will allow to point out candidates with a higher efficiency. The photometric characteristics of the secondary candidates seem very similar to those of the primary ones, although they are slightly redder and fainter. This indicates that the exact completeness of the present survey cannot be properly estimated without full identification of the secondary candidates. Comparison with other surveys suggests that the present one should potentially be fairly complete (for $z<2.2$ ) down to $B=19.0(U-B=-0.30)$ but it is definitely not complete in the range $B \in[19.0,19.6]$. This could be due to a bluer selection threshold in $U-B$ at these magnitudes.

We presented, for the different identified objects, information such as the nature of the object, its possible redshift, the line positions and equivalent widths, etc. Some of the objects have characteristics that deserve further investigation: besides the already studied associations Q0107 - 025A and B (Surdej et al. 1986) and Q0118 - 031A, B and C (Robertson et al. 1986), several quasars exhibit prominent line-of-sight absorption lines; one of the quasars is of BAL type.

We also investigated the spatial distribution of both the candidates and the confirmed quasars. We detected, for the quasars of our sample, a significant deviation from randomness in the form of a propensity to cluster in pairs on the celestial sphere with a typical scale of about 10 arcmin. We also noticed a deviation towards a threedimensional clustering attributable to the occurrence of only one very interesting pair of quasars having similar redshifts: Q0107 - 025A and B. The redshift distribution of the quasars in our sample exhibits one statistically marginal anomaly that manifests itself as an excess of quasars in the bin $z \in[0.9,1.0]$. This effect, if real, certainly deserves further investigation.

A survey, very similar to the one presented here, has been performed in a neighbouring field labelled NGC 520 (see Surdej et al. in preparation). This type of $U / B$ survey is, in fact, the oldest one and the precursor of our multitechnique quasar surveys.

Acknowledgements. This work was supported in part by contracts ARC 90/94-140 and 94/99-178 "Actions de Recherche Concertées de la Communauté Française", SC-005 "Services Fédéraux des Affaires Scientifiques, Techniques et Culturelles" (Belgium) and HCM network CHRX-CT92-0044 (European Union). O.M. was a European Space Agency fellow during his stay in Liège and he later received a financial support from the Société de Secours des Amis des Sciences (France). J.S. would like to acknowledge financial support from the FNRS (Belgium) for travel grants as well as PRODEX.

\section{Appendix}

Finding charts for the 63 (62 primary and one secondary) identified extragalactic objects in the catalogue (Table 6). The charts cover $2^{\prime} \times 2^{\prime}$; North is up, East is left. They correspond to a $B_{\mathrm{J}}$ band (made with the help of the Digitized Sky Survey).

\section{References}

Anderson N., Kunth D., Sargent W.L.W., 1988, AJ 95, 644

Anderson S.F., Margon B., 1987, ApJ 314, 111

Antonucci R., 1993, ARA\&A 31, 473

Aragón-Salamanca A., Ellis R.S., Schwartzenberg J.M., Bergeron J.A., 1994, ApJ 421, 27

Arp H.C., 1981, ApJ 250, 31

Arp H.C., 1983, in: Swings J.-P. (ed.) Proc. $24^{\text {th }}$ Liège Int. Astroph. Coll., Quasars and Gravitational Lenses, Université de Liège, p. 307 
Arp H.C., 1987, Quasars, Redshifts and Controversies. Interstellar Media, Berkeley

Arp H.C., Duhalde O., 1985, PASP 97, 1149

Arp H.C., Surdej J., 1982, A\&A 109, 101

Arp H.C., Bi H.G., Chu Y., Zhu X., 1990, A\&A 239, 33

Begelman M.C., 1989, in: Osterbrock D.E., Miller J.S. (eds.) Proc. IAU Symp. 134, Active Galactic Nuclei. Kluwer Acad. Publ., Dordrecht, p. 141

Berger J., Cordoni J.-P., Fringant A.-M., et al., 1991, A\&AS 87,389

Bergeron J., Boissé P., 1991, A\&A 243, 344

Blandford R.D., 1989, in: Osterbrock D.E., Miller J.S. (eds.) Proc. IAU Symp. 134, Active Galactic Nuclei. Kluwer Acad. Publ., Dordrecht, p. 233

Borgeest U., 1993, in: Surdej J. et al. (eds.) Proc. $31^{\text {st }}$ Liège Int. Astroph. Coll., Gravitational Lenses in the Universe, Université de Liège, p. 517

Boyle B.J., Fong R., Shanks T., Peterson B.A., 1987, MNRAS 227,717

Boyle B.J., Fong R., Shanks T., Peterson B.A., 1990, MNRAS 243,1

Braccesi A., Zitelli V., Bònoli F., Formiggini L., 1980, A\&A 85,80

Chaffee F.H., Foltz C.B., Hewett P.C., et al., 1991, AJ 102, 461

Clark P.J., Evans F.C., 1954, Ecology 35, 445

Clowes R.G., Campusano L.E., 1991a, MNRAS 249, 218

Clowes R.G., Campusano L.E., 1991b, PASPC 21, 248

Crampton D., Cowley A.P., Hartwick F.D.A., 1987, ApJ 314, 129

Crampton D., Cowley A.P., Hartwick F.D.A., 1989, ApJ 345, 59

Cristiani S., La Franca F., Andreani P., et al., 1991, PASPC 21,22

Danks A.C., 1983, ESO users' manual, ESO

de Lapparent V., Geller M.J., Huchra J.P., 1988, ApJ 332, 44

Ekers J.A., 1969, Aust. J. Phys. Astrophys. Suppl. 7, 1

Ferland G.J., 1981, ApJ 249, 17

Geller M.J., Huchra J.P., 1989, Sci 246, 897

Gosset E., 1987a, Analyse de nuages de points: applications astronomiques et étude de la distribution des quasars, Thèse de doctorat, Université de Liège

Gosset E., 1987b, A\&A 188, 258

Gosset E., Louis B., 1986, Ap\&SS 120, 263

Gosset E., Surdej J., Swings J.-P., 1986, in: Swarup G. and Kapahi V.K. (eds.) Proc. IAU Symp. 119, Quasars. Reidel, Dordrecht, p. 45

Gosset E., Surdej J., Swings J.-P., 1988, PASPC 2, 281

Gosset E., Clowes R.G., Surdej J., Swings J.-P., 1990, MNRAS 245,71

Gosset E., Surdej J., Cheng F.H., Swings J.-P., 1991, PASPC 21,84

Graham M.J., Clowes R.G., Campusano L.E., 1995, MNRAS 275,790

Guibert J., Moreau O., 1991, The ESO Messenger 64, 69

Iovino A., Shaver P.A., Cristiani S., 1991, PASPC 21, 202

Krolik J.H., 1989, in: Osterbrock D.E., Miller J.S. (eds.) Proc. IAU Symp. 134, Active Galactic Nuclei. Kluwer Acad. Publ., Dordrecht, p. 285

Landolt A.U., 1983, AJ 88, 439

Lynds C.R., 1967, ApJ 147, 837

McAlpine G.M., Feldman F.R., 1982, ApJ 261, 412
McAlpine G.M., Lewis D.W., 1978, ApJS 36, 587

Middelburg F., 1985, IHAP manual, ESO

Mo H.J., Fang L.Z., 1993, ApJ 410, 493

Moles M., Márquez I., Masegosa J., del Olmo A., Perea J., Arp H.C., 1994, ApJ 432, 135

Moreau O., 1992, Sélection de candidats quasars par analyse automatique de clichés photographiques, Thèse de doctorat, Université Paris 7

Moreau O., Reboul H., 1995, A\&AS 111, 169

Moreau O., Gosset E., Reboul H., Vanderriest C., 1994, in: MacGillivray H.T. et al. (eds.) Proc. of IAU Symp. 161, Astronomy from Wide-Field Imaging. Kluwer Acad. Publ., Dordrecht, p. 740

Netzer H., 1989, in: Osterbrock D.E., Miller J.S. (eds.) Proc. IAU Symp. 134, Active Galactic Nuclei. Kluwer Acad. Publ., Dordrecht, p. 69

Oke J.B., Gunn J.E., 1982, PASP 94, 586

Oort J.H., 1981, A\&A 94, 359

Oort J.H., 1983, in: Swings J.-P. (ed.) Proc. $24^{\text {th }}$ Liège Int. Astroph. Coll., Quasars and Gravitational Lenses, Université de Liège, p. 301

Oort J.H., Arp H.C., de Ruiter H., 1981, A\&A 95, 7

Osmer P.S., 1981, ApJ 247, 762

Partridge R.B., 1995, 3K: The Cosmic Microwave Background Radiation. Cambridge University Press

Peacock J.A., 1983, MNRAS 202, 615

Refsdal S., Surdej J., 1994, Rep. on Progress in Phys. 57, 117

Robertson J.G., Shaver P.A., Surdej J., Swings J.-P., 1986, MNRAS 219, 403

Rose J.A., 1977, ApJ 211, 311

Rosenfeld A., 1969, Picture Processing by Computer. Academic Press, New York

Röser S., Bastian U., 1991, PPM Star Catalogue. Astronomisches Rechen Institut Heidelberg

Sargent W.L.W., Boksenberg A., 1983, in: Swings J.-P. (ed.) Proc. $24^{\text {th }}$ Liège Int. Astroph. Coll., Quasars and Gravitational Lenses. Université de Liège, p. 518

Sargent W.L.W., Young P.J., Boksenberg A., Tytler D., 1980, ApJS 42, 41

Shanks T., Fong R., Boyle B.J., Peterson B.A., 1987, MNRAS 227,739

Sharp N.A., 1979, A\&A 74, 308

Shectman S.A., 1981, Carnegie Institution of Washington Year Book 80, 586

Smette A., 1994, Limits on the Ly- $\alpha$ cloud sizes based on spectra of gravitationally lensed quasars, Thèse de doctorat, Université de Liège

Smette A., Surdej J., Shaver P.A., et al., 1992, ApJ 389, 39

Smette A., Robertson J.G., Shaver P.A., et al., 1995, A\&AS 113,199

Smoot G.F., Bennett C.L., Kogut A., et al., 1992, ApJ 396, L1

Soubiran C., 1992, A\&A 259, 394

Stocke J.T., Morris S.L., Gioia I.M., et al., 1991, ApJS 76, 813

Surdej J., Swings J.-P., Arp H.C., Barbier R., 1982, A\&A 114, 182

Surdej J., Swings J.-P., Henry A., et al., 1983, in: Swings J.-P. (ed.) Proc. $24^{\text {th }}$ Liège Int. Astroph. Coll., Quasars and Gravitational Lenses, Université de Liège, p. 355

Surdej J., Arp H., Gosset E., et al., 1986, A\&A 161, 209

Surdej J., Moreau O., Swings J.-P., Gosset E. (in preparation) 
Swings J.-P., Arp H.C., Surdej J., Henry A., Gosset E., 1983, in: Swings J.-P. (ed.) Proc. $24^{\text {th }}$ Liège Int. Astroph. Coll., Quasars and Gravitational Lenses, Université de Liège, p. 37

Swings J.-P., Surdej J., Henry A., Gosset E., Arp H.C., 1985, Rev. Mex. Astron. Astrofís. 10, 91

Swings J.-P., Surdej J., Gosset E., 1988, PASPC 2, 61

Véron-Cetty M.-P., Véron P., 1989, ESO Sci. Rep. 7, European Southern Observatory

Véron-Cetty M.-P., Woltjer L., Véron P., 1988, A\&AS 76, 489

Vétois J., 1995, Traitement du Signal 12, 277
Wall J.V., Shimmins A.J., Merkelijn J.K., 1971, Aust. J. Phys. Astrophys. Suppl. 19, 1

Wampler E.J., Ponz D., 1985, ApJ 298, 448

Webster A.S., 1976a, MNRAS 175, 61

Webster A.S., 1976b, MNRAS 175, 71

Webster A.S., 1982, MNRAS 199, 683

Weedman D.W., 1986, Quasar Astronomy. Cambridge University Press (Astroph. Ser.)

Wills D., 1980, ApJ 240, 721

Wills D., Lynds R., 1978, ApJS 36, 317

Wills D., Netzer H., 1979, ApJ 233, 1 